\title{
Affine Birman-Wenzl-Murakami algebras and tangles in the solid torus
}

\author{
by \\ Frederick M. Goodman (Iowa City, IA) and \\ Holly Hauschild (La Jolla, CA)
}

Dedicated to William G. Bade on his 80th birthday

\begin{abstract}
The affine Birman-Wenzl-Murakami algebras can be defined algebraically, via generators and relations, or geometrically as algebras of tangles in the solid torus, modulo Kauffman skein relations. We prove that the two versions are isomorphic, and we show that these algebras are free over any ground ring, with a basis similar to a well known basis of the affine Hecke algebra.
\end{abstract}

\section{Contents}

1. Introduction $\quad 77$

2. The affine Kauffman tangle algebra $\quad 79$

3. The affine Birman-Wenzl-Murakami algebra 91

4. The affine Brauer algebra 103

5. Isomorphism of ordinary BMW and Kauffman tangle algebras 109

6. Isomorphism of affine BMW and Kauffman tangle algebras 126

$\begin{array}{ll}\text { References } & 136\end{array}$

\section{INTRODUCTION}

The purpose of this paper is to establish an isomorphism between the affine Birman-Wenzl-Murakami algebras (defined by generators and relations) and the algebras of $(n, n)$-tangles in the solid torus, modulo Kauffman skein relations.

The Birman-Wenzl-Murakami (or BMW) algebras were conceived in [2] and [11] as an algebraic framework for the Kauffman link invariant of [8]. The definition of these algebras by generators and relations was motivated

2000 Mathematics Subject Classification: 57M25, 81R50.

Key words and phrases: affine Birman-Wenzl-Murakami algebras, affine Kauffman tangle algebras. 
by certain canonical elements, and relations satisfied by these elements, in Kauffman tangle algebras in $D^{2} \times I$, that is, algebras of framed $(n, n)$-tangles in $D^{2} \times I$, modulo Kauffman skein relations. It is therefore not surprising, and is sometimes taken as evident, that the BMW algebras are isomorphic to the Kauffman tangle algebras. A proof of the isomorphism was given by Morton and Wassermann [10] in a paper from 1989 that unfortunately was never published. (A related result is given by Kauffman [8, Theorem 4.4]. See Remark 3.2 below.) Some aspects of the BMW algebras can be understood more clearly in the Kauffman tangle picture; for example, the existence of the Markov trace and conditional expectations is evident from the tangle picture, and an argument of Morton and Traczyk [9] gives the freeness of the Kauffman tangle algebras over an arbitrary ring.

The affine BMW algebras are related to the ordinary BMW algebras as the affine Hecke algebras of type $A$ are related to the ordinary Hecke algebras of type $A$. The affine algebras have an "extra" generator $x_{1}$ which satisfies the braid relation $x_{1} g_{1} x_{1} g_{1}=g_{1} x_{1} g_{1} x_{1}$ with the first "ordinary" braid generator $g_{1}$. The extra generator of the affine algebras can be imagined geometrically as a strand looping around the hole in $A \times I$, where $A$ denotes the annulus $S^{1} \times I$. The full set of relations for the affine BMW algebras (due to Häring-Oldenburg [5]) are modeled after relations which hold in the Kauffman tangle algebras in $A \times I$. So the affine BMW algebras "should" be isomorphic to the Kauffman tangle algebras in $A \times I$, assuming that a sufficient list of relations has been discovered. This isomorphism is our main result. On the way to proving the isomorphism, we also show that the affine BMW algebras, over any ring, are free with a basis generalizing a well known basis of the affine Hecke algebras.

As for the ordinary BMW algebras, certain properties of the affine BMW algebras are most easily obtained from the Kauffman tangle picture -in particular the existence of the Markov trace and conditional expectations. Moreover, we require the tangle picture even to describe our basis of the affine BMW algebras.

In proving our main theorems, we rely on the results of Morton and Wassermann for the ordinary BMW and Kauffman tangle algebras, as well as on techniques from their paper. Since their paper is not generally available, we have reproduced their results in Section 5 of the present paper.

The outline of the paper is as follows. In Sections 2 and 3 we introduce the ordinary and affine Birman-Wenzl-Murakami algebras and the Kauffman tangle algebra and derive basic properties of these algebras. In Section 4, we introduce an affine version of the Brauer centralizer algebra, which is a homomorphic image of the Kauffman tangle algebra in $A \times I$. Section 5 is devoted to the Morton-Wassermann proof of the isomorphism 
of the ordinary BMW and Kauffman tangle algebras. Finally, in Section 6 we obtain the main results of the paper.

In a future paper, we intend to study the cyclotomic BMW algebras, which are quotients of the affine BMW algebras in which the affine generator $x_{1}$ satisfies a polynomial relation. Cyclotomic BMW algebras have previously appeared in [4], [5], [15].

Let us mention some additional antecedents for the algebras studied here. The affine and cyclotomic Brauer algebras, which we use in Section 4, were introduced by Häring-Oldenburg [5], and the cyclotomic case was studied in [18]. Formally similar algebras determined by weighted Brauer diagrams are used in the approach of Olshanski and Okounkov to the representation theory of the infinite symmetric group; see, for example, [14], [13]. Nazarov introduced a "degenerate" affine BMW algebra, under the name "degenerate affine Wenzl algebra", in [12]. The cyclotomic version of this algebra was recently studied in [1]. Ram and Orellana [15] have studied certain representations of the affine BMW algebras.

We would like to thank Hans Wenzl for finding a significant error in a previous version of this paper.

\section{THE AFFINE KAUFFMAN TANGLE ALGEBRA}

In this section we introduce the geometric versions of the ordinary and affine Birman-Wenzl-Murakami algebras.

2.1. Framed tangles. The objects considered in this subsection, namely framed tangles in $S \times I$, where $S$ is an oriented surface, do not enter directly into the definition of the Kauffman tangle algebra and its affine counterpart, but they provide important motivation for the definition.

Let $S$ be a smooth oriented surface (with boundary). Let $I$ denote the unit interval $[0,1]$. Choose once and for all a countable family of mutually disjoint oriented intervals $\left\{J_{i}: i \in \mathbb{N}\right\}$ in $S$.

Definition 2.1. Fix integers $k, n \geq 0$. A framed $(k, n)$-tangle in $S \times I$ is a family of non-intersecting embedded piecewise smooth rectangles (ribbons) in $S \times I$ such that:

(1) with $F$ denoting the union of the ribbons,

$$
\begin{aligned}
F \cap \partial(S \times I) & =F \cap(S \times\{0,1\}) \\
& =J_{1} \times\{0,1\} \cup \cdots \cup J_{k} \times\{0,1\},
\end{aligned}
$$

(2) any ribbon in the family intersects the boundary of $S \times I$ transversally. 
REMARK 2.2. Note that $k=0$, or $n=0$, or both, are allowed in the definition of tangles. If one of $k$ or $n$ is positive, then a $(k, n)$-tangle must be a non-empty family of ribbons, but the empty tangle, with no ribbons, is an allowable $(0,0)$-tangle.

We will identify framed tangles which are isotopic via an isotopy fixing the intersections of the curves with the boundary of $S \times I$; so strictly speaking, a tangle is an isotopy class of families of ribbons.

One can compose $(k, n)$-tangles and $(n, m)$-tangles by "stacking". Namely, the first tangle (the $(k, n)$-tangle) is placed above the second tangle (the ( $n, m)$-tangle), the $n$ oriented intervals at which the ribbons intersect the lower boundary of the first tangle are identified with the $n$ oriented intervals at which the ribbons intersect the upper boundary of the second tangle, and the resulting family of ribbons is compressed into $S \times I$.

Since composition of tangles is evidently associative, framed tangles in $S \times I$ may be regarded as morphisms in a certain category. Namely, the objects of the category are $\{0,1, \ldots\}$, and the morphisms from $k$ to $n$ are the $(k, n)$-tangles in $S \times I$. In particular, the set of $(n, n)$-tangles forms a monoid, whose identity is the tangle $J_{1} \times I \cup \cdots \cup J_{n} \times I$.

We are interested here in two possibilities for $S$, namely the plane $\mathbb{R}^{2}$ and the annulus $A^{2}=\left\{(x, y) \in \mathbb{R}^{2}: 1 / 4 \leq x^{2}+y^{2}\right\}$. We refer to tangles in $\mathbb{R}^{2} \times I$ as "ordinary" framed tangles and tangles in $A^{2} \times I$ as "affine" framed tangles. For both cases we take our intervals $J_{i}$ to lie on the positive $x$-axis and to be ordered by their order on the $x$-axis. For convenience, we can take $J_{i}=[i, i+1 / 2] \times\{0\}$.

2.2. Tangle diagrams. Fix points $a_{i}$ in $\mathbb{R}$, for $i \geq 0$, with $0=a_{0}<$ $a_{1}<a_{2} \cdots$.

DEFINITION 2.3. An ordinary $(k, n)$-tangle diagram is a piece of a knot diagram in $R=\mathbb{R} \times I$ such that:

(1) with $F$ denoting the union of the curves comprising the knot diagram,

$$
\begin{aligned}
F \cap \partial(R) & =F \cap(I \times\{0,1\}) \\
& =\left\{\left(a_{0}, 1\right), \ldots,\left(a_{k-1}, 1\right)\right\} \cup\left\{\left(a_{0}, 0\right), \ldots,\left(a_{n-1}, 0\right)\right\},
\end{aligned}
$$

(2) any curve in the family intersects the boundary of $R$ transversally.

Recall that a knot diagram means a collection of piecewise smooth curves which may have intersections and self-intersections, but only simple transverse intersections. At each intersection or crossing, one of the two strands (pieces of curves) which intersect is indicated as crossing over the other. 
Definition 2.4. Two ordinary tangle diagrams are said to be ambient isotopic if they are related by a sequence of Reidemeister moves of types I, II, and III, followed by an isotopy of $R$ fixing the boundary. Two ordinary tangle diagrams are said to be regularly isotopic if they are related by a sequence of Reidemeister moves of types II and III only, followed by an isotopy of $R$ fixing the boundary. See the following figure for the Reidemeister moves of types I, II, and III.

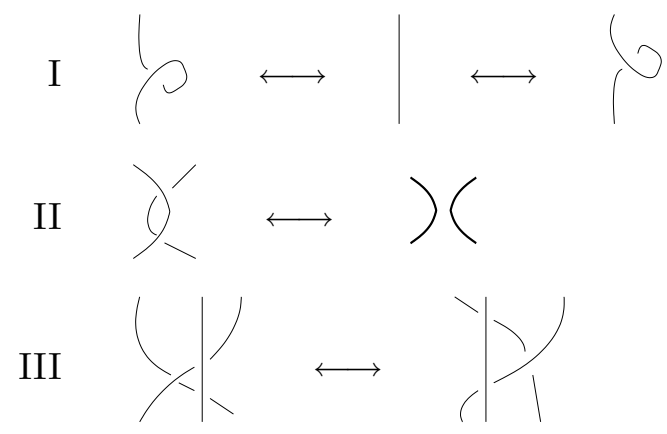

Tangle diagrams can be composed, similarly to tangles, and composition respects ambient isotopy or regular isotopy. Thus one obtains a product on ambient isotopy classes of tangle diagrams, respectively on regular isotopy classes of tangle diagrams.

As is well known, isotopy classes of ordinary framed tangles correspond bijectively to regular isotopy classes of ordinary tangle diagrams, by a framed version of Reidemeister's theorem. The correspondence is as follows: A representative of an isotopy class of framed tangles (in $(x, y, z)$-space) can always be chosen that lies close to and almost parallel with the $x z$ plane; twists in ribbons are converted to "kinks" (or almost planar loops) in such a representative:

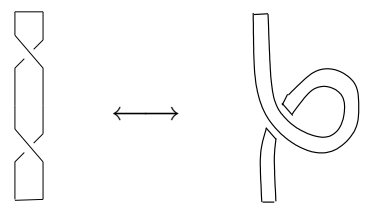

Replace each ribbon in such a representative by its "core", a curve running lengthwise along the center of the ribbon, and project the collection of these curves in the $x z$ plane. The result is a tangle diagram. Any two such projections are regularly isotopic tangle diagrams. Thus one has a well defined map from isotopy classes of ordinary framed tangles to regular isotopy classes of tangle diagrams. This map is bijective.

It follows that the monoid of (isotopy classes of) ordinary $(n, n)$-framed tangles is isomorphic to the monoid of regular isotopy classes of $(n, n)$-tangle diagrams. 
Definition 2.5. An affine $(k, n)$-tangle diagram is an ordinary $(k+1$, $n+1)$-tangle diagram which includes a distinguished curve $P$ connecting $\left(a_{0}, 1\right)$ and $\left(a_{0}, 0\right)$ such that the height coordinate (the second coordinate) varies monotonically along the curve $P$.

Note that an affine tangle diagram is always equivalent by an isotopy of $R$ fixing the boundary to a diagram including the curve $\left\{a_{0}\right\} \times I$. We will draw affine tangle diagrams with the distinguished curve drawn as a thickened vertical segment. We refer to the distinguished curve as the "flagpole". The figure below shows some fundamental affine tangle diagrams from which more complex affine tangle diagrams can be built by composition.

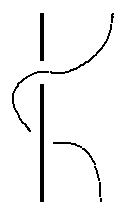

$X_{1}$

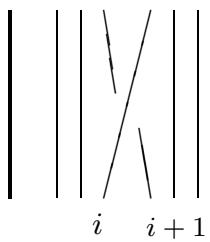

$G_{i}$

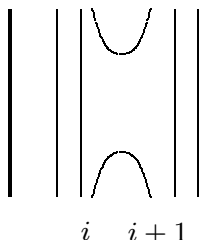

$E_{i}$

Two affine $(k, n)$-tangle diagrams are ambient (respectively regularly) isotopic if they are ambient (resp. regularly) isotopic as ordinary $(k+1, n+1)$ tangle diagrams. Note that in an ambient isotopy, no Reidemeister move of type I is ever applied to the flagpole, as the flagpole is required to be represented by a monotonic path.

The set of affine tangle diagrams, regarded as a subset of ordinary tangle diagrams, is evidently closed under the composition of tangle diagrams. Thus one obtains a product on ambient isotopy classes of affine tangle diagrams, respectively on regular isotopy classes of affine tangle diagrams.

We can extend the correspondence between isotopy classes of ordinary framed tangles and regular isotopy classes of ordinary tangle diagrams to a correspondence between isotopy classes of affine framed tangles and regular isotopy classes of affine tangle diagrams, as follows. First for each affine $(k, n)$-framed tangle $T$ in $A^{2} \times I$, we can consider $T \cup(\{0\} \times I)$ in $\mathbb{R}^{2} \times I$. The latter projects to an ordinary $(k+1, n+1)$-tangle with distinguished curve $P=\{0\} \times I$.

It follows that the monoid of (isotopy classes of) affine $(n, n)$-tangles is isomorphic to the monoid of regular isotopy classes of affine $(n, n)$-tangle diagrams.

2.3. The ordinary Kauffman tangle algebras. Let $\mathcal{U}(k, n)$ denote the family of ordinary $(k, n)$-tangle diagrams modulo regular isotopy. Likewise, let $\widehat{\mathcal{U}}(k, n)$ denote the family of affine $(k, n)$-tangle diagrams modulo regular isotopy. Then $\mathcal{U}(n, n)$ and $\widehat{\mathcal{U}}(n, n)$ are monoids for each $n$. 
Definition 2.6. Let $R$ be any (commutative, unital) ring with distinguished elements $\lambda, z$ and $\delta$, with $\lambda$ and $\delta$ invertible, satisfying the relation

$$
\lambda^{-1}-\lambda=z(\delta-1) .
$$

The (ordinary) Kauffman tangle algebra $\mathrm{KT}_{n, R}$ over $R$ is the monoid algebra $R \mathcal{U}(n, n)$ modulo the following relations:

(1) (Kauffman skein relation)

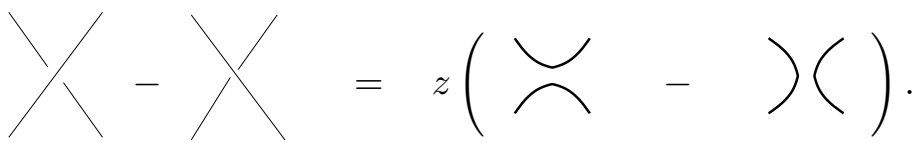

Here, the figures indicate tangle diagrams which differ only in the region shown and are identical outside this region.

(2) (Untwisting relation)

$$
\rho=\lambda \mid \text { and } \bigcirc=\lambda^{-1}
$$

(3) (Free loop relation)

$$
T \cup \bigcirc=\delta T,
$$

where $T \cup \bigcirc$ is a tangle diagram consisting of the union of $T$ and an additional closed loop having no crossing with $T$ and no selfcrossings.

The generic Kauffman tangle algebra (as considered by Morton and Traczyk [9] and Morton and Wassermann [10]) is the Kauffman tangle algebra $\mathrm{KT}_{n, \Lambda}$ over the ring

$$
\Lambda=\mathbb{Z}\left[\boldsymbol{\lambda}^{ \pm 1}, \boldsymbol{z}, \boldsymbol{\delta}^{ \pm 1}\right] /\left\langle\boldsymbol{\lambda}^{-1}-\boldsymbol{\lambda}=\boldsymbol{z}(\boldsymbol{\delta}-1)\right\rangle .
$$

Here $\boldsymbol{\lambda}, \boldsymbol{z}, \boldsymbol{\delta}$ are indeterminates. For any ring $R$ as above, there is a homomorphism from $\Lambda$ to $R$ determined by $\boldsymbol{\lambda} \mapsto \lambda, z \mapsto z$, and $\boldsymbol{\delta} \mapsto \delta$. Thus the specialization $\mathrm{KT}_{n, \Lambda} \otimes_{\Lambda} R$ makes sense. It follows from the freeness of Kauffman tangle algebras [10] that for any $R$,

$$
\mathrm{KT}_{n, R} \cong \mathrm{KT}_{n, \Lambda} \otimes_{\Lambda} R ;
$$

see Corollary 5.9 below. This is actually a special case of a general universal coefficient theorem for skein modules due to J. Przytycki; see [17, Lemma 5].

REMARK 2.7. If $R$ is a ring with distinguished elements $\lambda, z$, and $\delta$ as above, and $S \supseteq R$ is a ring containing $R$, then the inclusion

$$
R \mathcal{U}(n, n) \rightarrow S \mathcal{U}(n, n)
$$

induces an $R$-algebra homomorphism 


$$
\mathrm{KT}_{n, R} \rightarrow \mathrm{KT}_{n, S}
$$

This map is always injective; see Corollary 5.10.

2.4. The affine Kauffman tangle algebras. Let $R$ be a ring with distinguished elements $\lambda, z$ and $\delta$ as above. Our preliminary definition of the affine Kauffman tangle algebra over $R$ is the monoid algebra $R \widehat{\mathcal{U}}(n, n)$ modulo the Kauffman skein relation, the untwisting relation, and the free loop relation, as for the ordinary Kauffman tangle algebras. Here it is understood that none of the curves in the diagrams for these relations represent a part of the flagpole. Denote this algebra temporarily by $\widehat{K}_{n, R}$.

For $r \geq 1$, let $\Theta_{r}$ (resp. $\Theta_{-r}$ ) denote the (regular isotopy class of) the closed curve with no self-crossings that winds $r$ times around the flagpole in the positive sense (resp. in the negative sense).

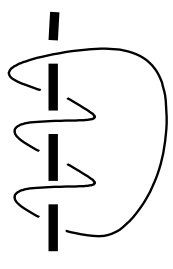

$\Theta_{3}$

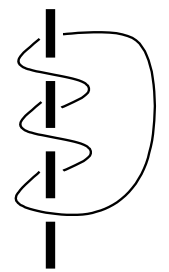

$\Theta_{-3}$

It is not difficult to see that the curves $\Theta_{r}$ generate $\widehat{K}_{0, R}$. A theorem of Turaev [19] says that $\left\{\Theta_{r}: r \geq 1\right\}$ is algebraically independent in $\widehat{K}_{0, \Lambda}$. Hence $\widehat{K}_{0, \Lambda}$ is the polynomial algebra over $\Lambda$ in the infinitely many variables $\Theta_{r}$ for $r \geq 1$.

For any $R$, one has an algebra map from $\widehat{K}_{0, R}$ to $\widehat{K}_{0, \Lambda} \otimes_{\Lambda} R$; since the former is generated by $\left\{\Theta_{r}: r \geq 1\right\}$ and the latter is the polynomial algebra over $R$ in the variables $\left\{\Theta_{r}: r \geq 1\right\}$, it follows that the map is an isomorphism. Thus, $\widehat{K}_{0, R}$ is the polynomial algebra over $R$ in the variables $\Theta_{r}$ for $r \geq 1$.

We have $\Theta_{1}=\Theta_{-1}$ in $\widehat{K}_{0, R}$, but $\Theta_{r}$ is not ambient isotopic to $\Theta_{-r}$ for $r \geq 2$. According to Turaev's theorem, $\Theta_{-r}$ is a polynomial in the variables $\Theta_{k}, k \geq 1$,

$$
\Theta_{-r}=f_{r}\left(\Theta_{1}, \Theta_{2}, \ldots\right) .
$$

Later we will need a little more information about the polynomials $f_{r}$. For $a \geq 1, b \geq 0$, let $\Theta_{a, b}$ be the curve with $a$ positive windings around the flagpole, and one positive crossing, and $b$ negative windings, as in the first of the following figures. Let $\Theta_{a, b}^{-}$be the curve with the crossing reversed. 

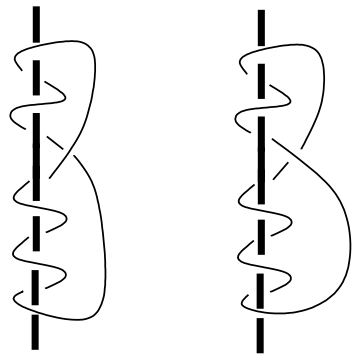

$\Theta_{2,3}$

$\Theta_{2,3}^{-}$

In particular, $\Theta_{r, 0}=\lambda^{-1} \Theta_{r}$; we interpret $\Theta_{0}$ as $\delta$.

LEMMA 2.8. Let $r, a, b \geq 1$.

(1) $\Theta_{-r}=\lambda \Theta_{1, r-1}$.

(2) $\Theta_{a, b}=\lambda^{2} \Theta_{a+1, b-1}+z\left(\Theta_{a} \Theta_{-b}-\Theta_{a-b}\right)$.

(3) $f_{r}\left(\Theta_{1}, \Theta_{2}, \ldots\right)=\lambda^{2 r-2} \Theta_{r}+z f_{r}^{\prime}\left(\Theta_{1}, \ldots, \Theta_{r-1}\right)$, where $f_{r}^{\prime}$ is a polynomial in $\Theta_{1}, \ldots, \Theta_{r-1}$.

Proof. Point (1) follows from introducing a twist at the top of $\Theta_{-r}$ :

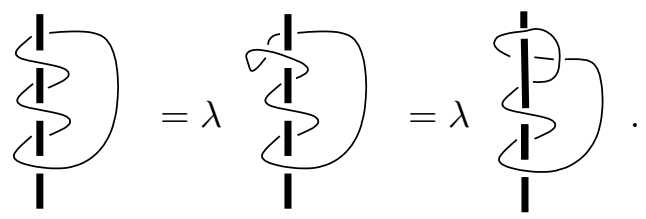

The tangle obtained by smoothing the crossing in $\Theta_{a, b}$ horizontally is $\Theta_{a} \Theta_{-b}$, and the tangle obtained by smoothing the crossing vertically is $\Theta_{a-b}$, while $\Theta_{a, b}^{-}=\lambda^{2} \Theta_{a+1, b-1}$. Thus the Kauffman skein relation gives

$$
\Theta_{a, b}=\lambda^{2} \Theta_{a+1, b-1}+z\left(\Theta_{a} \Theta_{-b}-\Theta_{a-b}\right) .
$$

An induction based on points (1) and (2) yields (3).

We now return to the definition of the affine Kauffman tangle algebra. Because $\widehat{K}_{0, R}$ is a polynomial algebra over $R$ and $\widehat{K}_{n, R}$ is a $\widehat{K}_{0, R}$ module, it makes sense to just absorb $\widehat{K}_{0, R}$ into the ground ring for the affine Kauffman tangle algebra, and this is what we do.

Definition 2.9. Let $S$ be a ring containing distinguished elements $\lambda, z$, $\delta$, and $q_{r}(r \geq 1)$, with $\lambda$ and $\delta$ invertible, such that the relation $\lambda^{-1}-\lambda=$ $z(\delta-1)$ holds. The affine Kauffman tangle algebra

$$
\widehat{\mathrm{KT}}_{n, S}=\widehat{\mathrm{KT}}_{n, S}\left(\lambda, z, \delta, q_{1}, q_{2}, \ldots\right)
$$

is the monoid algebra $S \widehat{\mathcal{U}}(n, n)$ modulo the following relations: 
(1) The Kauffman skein relation, the untwisting relations, and the free loop relation, as for the ordinary Kauffman tangle algebra.

(2) $T \cup \Theta_{r}=q_{r} T$, where $T \cup \Theta_{r}$ is the union of a tangle $T$ and a copy of the curve $\Theta_{r}$, such that there are no crossings between $T$ and $\Theta_{r}$.

REMARK 2.10. Note that $\widehat{\mathrm{KT}}_{0, S} \cong S$.

If $R$ is a ring with distinguished elements $\lambda, z$, and $\delta$ as above, and $\boldsymbol{q}_{r}$, $r \geq 1$, are indeterminates, we denote by $\widehat{R}$ the polynomial ring $R\left[\boldsymbol{q}_{1}, \boldsymbol{q}_{2}, \ldots\right]$.

REMARK 2.11. Let $R$ be a ring with distinguished elements $\lambda, z$, and $\delta$ as above, and $S \supseteq R$ a ring with additional elements $q_{1}, q_{2}, \ldots$ Adding a flagpole to ordinary $(n, n)$-tangle diagrams induces a homomorphism of $R$-algebras $i_{n}: \mathrm{KT}_{n, R} \rightarrow \widehat{\mathrm{KT}}_{n, S}$. This homomorphism is always injective. For the moment, we verify injectivity in two special cases.

(1) $i_{n}: \mathrm{KT}_{n, S} \rightarrow \widehat{\mathrm{KT}}_{n, S}$ is injective. In fact the image of $i_{n}$ is the span of affine $(n, n)$-tangle diagrams having no intersection with the flagpole. We can define a map $o_{n}$ of such diagrams to ordinary $(n, n)$-tangle diagrams by removing the flagpole. The map $o_{n}$ induces an $S$-algebra homomorphism inverse to $i_{n}$; that is, $o_{n} \circ i_{n}$ is the identity on $\mathrm{KT}_{n, S}$.

(2) $i_{n}: \mathrm{KT}_{n, R} \rightarrow \widehat{\mathrm{KT}}_{n, \widehat{R}}$ is injective. In fact, removing the flagpole from affine $(n, n)$-tangle diagrams and mapping $\boldsymbol{q}_{r} \mapsto \delta$ determines a homomorphism of $R$-algebras $f_{n}: \widehat{\mathrm{KT}}_{n, \widehat{R}} \rightarrow \mathrm{KT}_{n, R}$, and $f_{n} \circ i_{n}$ is the identity on $\mathrm{KT}_{n, R}$.

Once we have verified that $\mathrm{KT}_{n, R}$ imbeds in $\mathrm{KT}_{n, S}$ (see Corollary 5.10), it will follow from (1) that $\mathrm{KT}_{n, R}$ imbeds in $\widehat{\mathrm{KT}}_{n, S}$ in general.

The generic affine Kauffman tangle algebra is the Kauffman tangle algebra

$$
\widehat{\mathrm{KT}}_{n, \widehat{\Lambda}}=\widehat{\mathrm{KT}}_{n, \widehat{\Lambda}}\left(\boldsymbol{\lambda}, \boldsymbol{z}, \boldsymbol{\delta}, \boldsymbol{q}_{1}, \boldsymbol{q}_{2}, \ldots\right)
$$

over the ring $\hat{\Lambda}=\Lambda\left[\boldsymbol{q}_{1}, \boldsymbol{q}_{2}, \ldots\right]$.

Since for any ring $S$ as above, we have a homomorphism $\hat{\Lambda}$ to $S$, determined by the assignments $\boldsymbol{\lambda} \mapsto \lambda, z \mapsto z, \boldsymbol{\delta} \mapsto \delta$, and $\boldsymbol{q}_{i} \mapsto q_{i}$, the specialization $\widehat{\mathrm{KT}}_{n, \widehat{\Lambda}} \otimes_{\widehat{\Lambda}} S$ makes sense. We will eventually show that

$$
\widehat{\mathrm{KT}}_{n, S} \cong \widehat{\mathrm{KT}}_{n, \widehat{\Lambda}} \otimes_{\widehat{\Lambda}} S
$$

see Corollary 6.14.

REMARK 2.12. We will use the following specialization below, in connection with an affine version of the Brauer algebra: Set $R=\mathbb{Z}\left[\delta^{ \pm 1}\right]$. We have a homomorphism of $e: \Lambda \rightarrow R$ determined by $\lambda \mapsto 1, z \mapsto 0, \delta \mapsto \delta$. Then $\widehat{\mathrm{KT}}_{n, \widehat{R}}$ is the quotient of $\widehat{R} \widehat{\mathcal{U}}(n, n)$ by the relations: 
(1) (Kauffman skein relation)

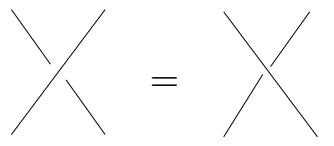

for a crossing of strands neither of which represents a part of the flagpole.

(2) (Untwisting relation)

$$
\rho=\mid \text { and } \rho=
$$

(3) (Removal of closed loops) $T \cup \bigcirc=\delta T$, where $T \cup \bigcirc$ is a tangle diagram consisting of the union of $T$ and an additional closed loop having no crossings with the flagpole, and $T \cup \Theta_{r}=\boldsymbol{q}_{r} T$, for $r \geq 1$.

Since in this specialization, two tangle diagrams related by crossing changes are equivalent, for any $r \geq 1$ and for any closed curve $c$ which loops $r$ times around the flagpole, $c=\Theta_{r}=\boldsymbol{q}_{r}$.

2.5. The elements $X_{r}$. We define elements $X_{r} \in \widehat{\mathrm{KT}}_{n, S}$ for $1 \leq r \leq n$ by

$$
X_{r}=\left(G_{r-1} \cdots G_{2} G_{1}\right) X_{1}\left(G_{1} G_{2} \cdots G_{r-1}\right) .
$$

For example,

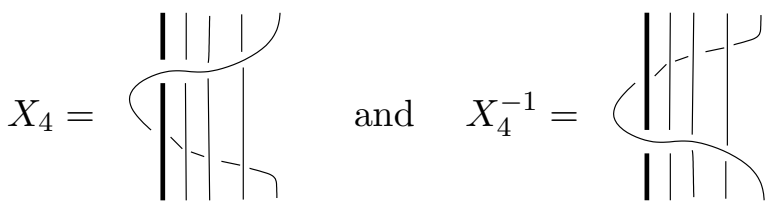

2.6. Symmetries. The map on ordinary (respectively, affine) tangle diagrams which flips diagrams top to bottom induces an anti-automorphism $\alpha$ of $\mathrm{KT}_{n, R}$ (respectively, of $\widehat{\mathrm{KT}}_{n, S}$ ). The anti-automorphism $\alpha$ fixes $E_{i}, G_{i}$, and $X_{1}$.

There is an isomorphism $\beta: \mathrm{KT}_{n, R}(\lambda, z, \delta) \rightarrow \mathrm{KT}_{n, R}\left(\lambda^{-1},-z, \delta\right)$ determined by the map of ordinary tangle diagrams that reverses all crossings.

For $r \geq 1$, define $q_{-r}=f_{r}\left(q_{1}, \ldots, q_{r}\right)$, where $f_{r}$ is the polynomial of equation (2.1). There is an isomorphism

$$
\beta: \widehat{\mathrm{KT}}_{n, S}\left(\lambda, z, \delta, q_{1}, q_{2}, \ldots\right) \rightarrow \widehat{\mathrm{KT}}_{n, S}\left(\lambda^{-1},-z, \delta, q_{-1}, q_{-2}, \ldots\right)
$$

determined by the map of affine tangle diagrams that reverses all crossings (also crossings of ordinary strands with the flagpole).

The ordinary Kauffman tangle algebra $\mathrm{KT}_{n, R}$ has an automorphism $\varrho_{n}$ which flips ordinary tangle diagrams left to right. The automorphism $\varrho_{n}$ satisfies $\varrho_{n}\left(E_{i}\right)=E_{n+1-i}$ and $\varrho_{n}\left(G_{i}\right)=G_{n+1-i}$. 
2.7. Inclusions, conditional expectations, and trace. The observations in this section apply equally to the ordinary and affine Kauffman tangle algebras. We fix a ring $S$ containing distinguished elements $\lambda, z, \delta$, and $q_{r}(r \geq 1)$ as above, and we let $\widehat{\mathrm{KT}}_{n}$ denote $\widehat{\mathrm{KT}}_{n, S}$.

For $n \geq 2$, the map $\iota$ from affine $(n-1, n-1)$-tangle diagrams to affine $(n, n)$-tangle diagrams that adds an additional strand on the right without adding any crossings:

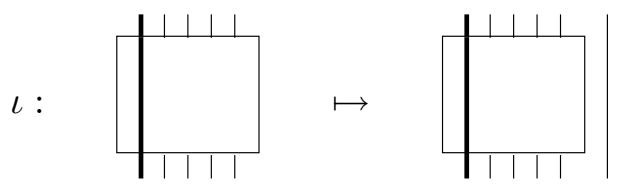

respects regular isotopy, composition of tangle diagrams, and the relations of the affine Kauffman tangle algebras, so induces a homomorphism $\iota$ : $\widehat{\mathrm{KT}}_{n-1} \rightarrow \widehat{\mathrm{KT}}_{n}$. Note that $\alpha \circ \iota=\iota \circ \alpha$, where $\alpha$ is the anti-automorphism of the affine Kauffman tangle algebras described in Section 2.6.

The map of affine $(n, n)$-tangle diagrams to affine $(n-1, n-1)$-tangle diagrams that "closes" the rightmost strand, without adding any crossings:

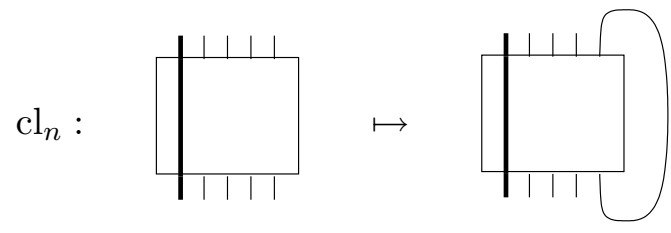

respects regular isotopy and the relations of the affine Kauffman tangle algebras, so induces an $S$-linear map $\widehat{\mathrm{KT}}_{n} \rightarrow \widehat{\mathrm{KT}}_{n-1}$. We define $\varepsilon_{n}: \widehat{\mathrm{KT}}_{n} \rightarrow$ $\widehat{\mathrm{KT}}_{n-1}$ by

$$
\varepsilon_{n}(T)=\delta^{-1} \operatorname{cl}_{n}(T)
$$

Note that $\varepsilon_{n} \circ \alpha=\alpha \circ \varepsilon_{n}$, where $\alpha$ is the anti-automorphism of the affine Kauffman tangle algebras described above.

We have

$$
\varepsilon_{n} \circ \iota(x)=x
$$

for $x \in \widehat{\mathrm{KT}}_{n-1}$. In particular, the map $\iota$ of $\widehat{\mathrm{KT}}_{n-1}$ into $\widehat{\mathrm{KT}}_{n}$ is injective. Consequently, we will drop the notation $\iota$ and regard $\widehat{\mathrm{KT}}_{n-1}$ as a subalgebra of $\widehat{\mathrm{KT}}_{n}$.

More generally,

$$
\varepsilon_{n}(x y)=x \varepsilon_{n}(y), \quad \varepsilon_{n}(y x)=\varepsilon_{n}(y) x
$$


for $x \in \widehat{\mathrm{KT}}_{n-1}$ and $y \in \widehat{\mathrm{KT}}_{n}$; that is, $\varepsilon_{n}$ is a $\widehat{\mathrm{KT}}_{n-1}-\widehat{\mathrm{KT}}_{n-1}$-bimodule map, or conditional expectation.

REMARK 2.13. One has the formula

$$
E_{n} x E_{n}=\operatorname{cl}_{n}(x) E_{n}
$$

for $x$ an (affine) $(n, n)$-tangle diagram. Equivalently, the idempotent $E_{n} / \delta$ implements the conditional expectation:

$$
\left(E_{n} / \delta\right) x\left(E_{n} / \delta\right)=\varepsilon_{n}(x)\left(E_{n} / \delta\right)
$$

for $x \in \widehat{\mathrm{KT}}_{n}$.

We define $\varepsilon: \widehat{\mathrm{KT}}_{n} \rightarrow \widehat{\mathrm{KT}}_{0} \cong S$ by

$$
\varepsilon=\varepsilon_{1} \circ \cdots \circ \varepsilon_{n} .
$$

Equivalently, if we define the closure of an affine $(n, n)$-tangle diagram to be the affine $(0,0)$-diagram obtained by closing all strands without introducing any new crossings:
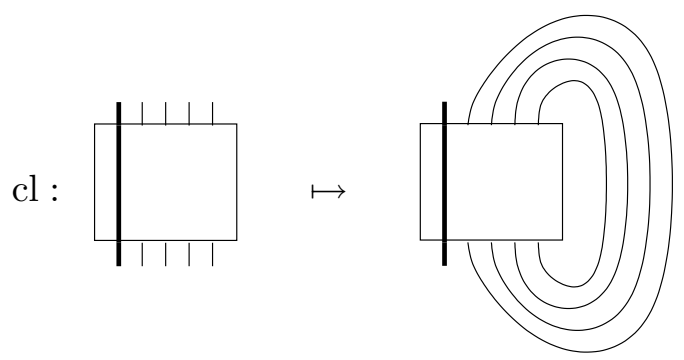

then $\varepsilon(x)=\delta^{-n} \operatorname{cl}(x)$. Note that

$$
\varepsilon \circ \iota=\varepsilon, \quad \varepsilon \circ \varepsilon_{n}=\varepsilon .
$$

We will show below that $\widehat{\mathrm{KT}}_{n}$ is generated as a unital algebra by $X_{1}^{ \pm 1}$, $G_{i}^{ \pm 1}$ and $E_{i}(1 \leq i \leq n-1)$. In particular $\widehat{\mathrm{KT}}_{1}$ is generated by $X_{1}^{ \pm 1}$, so is commutative.

For $n \geq 2$, let $\varepsilon_{n, 1}$ denote the map

$$
\varepsilon_{n, 1}=\varepsilon_{2} \circ \cdots \circ \varepsilon_{n}: \widehat{\mathrm{KT}}_{n} \rightarrow \widehat{\mathrm{KT}}_{1} .
$$

Then $\varepsilon_{n, 1}$ is a conditional expectation, so for $x \in \widehat{\mathrm{KT}}_{n}$,

$$
\varepsilon_{n, 1}\left(X_{1}^{ \pm 1} x\right)=X_{1}^{ \pm 1} \varepsilon_{n, 1}(x)=\varepsilon_{n, 1}(x) X_{1}^{ \pm 1}=\varepsilon_{n, 1}\left(x X_{1}^{ \pm 1}\right) .
$$

Since $\varepsilon=\varepsilon_{1} \circ \varepsilon_{n, 1}$, we have

$$
\varepsilon\left(X_{1}^{ \pm 1} x\right)=\varepsilon\left(x X_{1}^{ \pm 1}\right) .
$$

For any affine $(n, n)$-tangle diagram $T$ and any $r \leq n-1$, the closure of the affine tangle diagram $E_{r} T$ is isotopic to the closure of the affine tangle 
diagram $T E_{r}$, and similarly for $E_{r}$ replaced by $G_{r}^{ \pm 1}$. The following figure illustrates this for $G_{r}^{-1}$ :

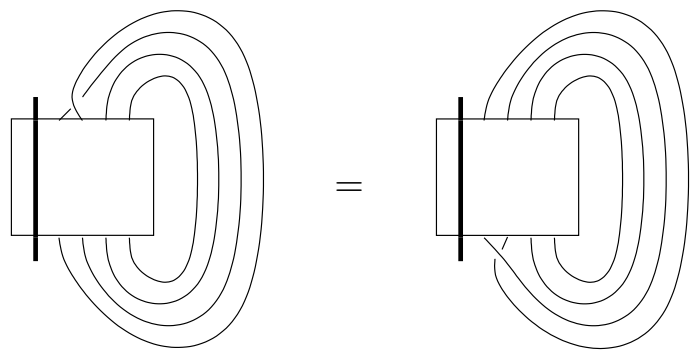

It follows that for $x \in \widehat{\mathrm{KT}}_{n}$ and $r \leq n-1$,

$$
\varepsilon\left(E_{r} x\right)=\varepsilon\left(x E_{r}\right), \quad \varepsilon\left(G_{r}^{ \pm 1} x\right)=\varepsilon\left(x G_{r}^{ \pm 1}\right)
$$

Proposition 2.14. $\varepsilon: \widehat{\mathrm{KT}}_{n} \rightarrow \widehat{\mathrm{KT}}_{0} \cong S$ is a trace. That is, $\varepsilon$ is $S$-linear and $\varepsilon(x y)=\varepsilon(y x)$ for all $x, y \in \widehat{\mathrm{KT}}_{n}$.

Proof. We have shown that the trace property

$$
\varepsilon(x y)=\varepsilon(y x)
$$

holds when $y$ is arbitrary and $x$ is one of $X_{1}^{ \pm 1}, G_{i}^{ \pm 1}$ and $E_{i}(1 \leq i \leq n-1)$. It follows that the trace property also holds when $x$ is any product of the elements $X_{1}^{ \pm 1}, G_{i}^{ \pm 1}$ and $E_{i}$. Since these elements generate $\widehat{\mathrm{KT}}_{n}$ as a unital algebra, the property holds for all $x$.

REMARK 2.15 . One easily checks by picture proofs that $\varepsilon_{r+1}\left(T G_{r}^{ \pm 1}\right)=$ $\left(\lambda^{ \pm 1} / \delta\right) T$, and hence

$$
\varepsilon\left(T G_{r}^{ \pm 1}\right)=\varepsilon(T) \lambda^{ \pm 1} / \delta
$$

for $T \in \widehat{\mathrm{KT}}_{r}$. Likewise, $\varepsilon_{r+1}\left(T E_{r}\right)=(1 / \delta) T$, and hence

$$
\varepsilon\left(T E_{r}\right)=\varepsilon(T) / \delta
$$

for $T \in \widehat{\mathrm{KT}}_{r}$. Moreover, if $X_{r}^{\prime}$ denotes

$$
X_{r}^{\prime}=G_{r-1} \cdots G_{1} X_{1} G_{1}^{-1} \cdots G_{r-1}^{-1},
$$

then for $s \geq 1$ and $T \in \widehat{\mathrm{KT}}_{r}$, one has $\varepsilon_{r+1}\left(T\left(X_{r}^{\prime}\right)^{s}\right)=q_{s} T$. Consequently,

$$
\varepsilon\left(T\left(X_{r}^{\prime}\right)^{s}\right)=q_{s} \varepsilon(T) .
$$

It follows from Lemma 2.8 that

$$
\varepsilon\left(T\left(X_{r}^{\prime}\right)^{-s}\right)=f_{s}\left(q_{1}, \ldots, q_{s}\right) \varepsilon(T) .
$$

A trace with these properties is usually called a Markov trace. The terminology originated in [6]. 


\section{THE AFFINE BIRMAN-WENZL-MURAKAMI ALGEBRA}

3.1. The Birman-Wenzl-Murakami algebra. The Birman-WenzlMurakami algebras were introduced independently by Birman and Wenzl [2] and by Murakami [11] as an algebraic setting for the Kauffman link invariant [8]. These algebras are known to appear as centralizer algebras for the quantum universal enveloping algebras of $\operatorname{sp}(2 n, \mathbb{C})$ or $\operatorname{so}(n, \mathbb{C})$ acting on tensor powers of the vector representation. They are deformations of Brauer's centralizer algebras (see [20]), and are extensions of the Hecke algebras of type $A$, as will be explained below.

The presentation which we give here follows Morton and Wassermann [10]. The parameters differ slightly from those used by Birman and Wenzl.

As before, let $R$ be a commutative unital ring with invertible elements $\lambda$ and $\delta$ and an element $z$ satisfying

$$
\lambda^{-1}-\lambda=z(\delta-1) .
$$

Definition 3.1. The Birman-Wenzl-Murakami algebra $W_{n, R}$ is the $R$ algebra with generators $g_{i}^{ \pm 1}$ and $e_{i}(1 \leq i \leq n-1)$ and relations:

(1) (Inverses) $g_{i} g_{i}^{-1}=g_{i}^{-1} g_{i}=1$.

(2) (Idempotent relation) $e_{i}^{2}=\delta e_{i}$.

(3) (Braid relations) $g_{i} g_{i+1} g_{i}=g_{i+1} g_{i} g_{i+1}$ and $g_{i} g_{j}=g_{j} g_{i}$ if $|i-j| \geq 2$.

(4) (Commutation relations) $g_{i} e_{j}=e_{j} g_{i}$ and $e_{i} e_{j}=e_{j} e_{i}$ if $|i-j| \geq 2$.

(5) (Tangle relations) $e_{i} e_{i \pm 1} e_{i}=e_{i}, g_{i} g_{i \pm 1} e_{i}=e_{i \pm 1} e_{i}$, and $e_{i} g_{i \pm 1} g_{i}=$ $e_{i} e_{i \pm 1}$.

(6) (Kauffman skein relation) $g_{i}-g_{i}^{-1}=z\left(e_{i}-1\right)$.

(7) (Untwisting relations) $g_{i} e_{i}=e_{i} g_{i}=\lambda^{-1} e_{i}$, and $e_{i} g_{i \pm 1} e_{i}=\lambda e_{i}$.

REMARK 3.2.

(1) If $z$ is taken to be invertible, then

$$
e_{i}=1+z^{-1}\left(g_{i}^{-1}-g_{i}\right) \text {. }
$$

In this case, several of the relations are redundant. Moreover, the algebra is a quotient of the braid group algebra.

(2) In the parametrization of [21], $z$ is replaced by $q-q^{-1}$, where $q$ is another indeterminate, and $q-q^{-1}$ is assumed to be invertible. The Kauffman skein relation then becomes a cubic relation for the braid generators $g_{i}$.

(3) The main result of [10] is that the Birman-Wenzl-Murakami algebra $W_{n, \Lambda}$ defined over $\Lambda$ is isomorphic to the Kauffman tangle algebra $\mathrm{KT}_{n, \Lambda}$.

In [8], Theorem 4.4, Kauffman gives a presentation for the algebra of tangles generated by the $E_{i}$ 's, $G_{i}$ 's, and free loops. It is not clear to us how this result is related to the Morton-Wassermann theorem. 
REMARK 3.3.

(1) The assignment $e_{i} \mapsto e_{i}, g_{i} \mapsto g_{i}$ defines a homomorphism $\iota$ from $W_{n, R}$ to $W_{n+1, R}$, since the relations are preserved. It is not evident that $\iota$ is injective, but this will follow eventually from the isomorphism of $W_{n, R}$ with $\mathrm{KT}_{n, R}$; see Theorem 5.41.

(2) Define $S(x): W_{n, R} \rightarrow W_{n+1, R}$ by

$$
S(x)=\operatorname{Ad}\left(g_{n} g_{n-1} \cdots g_{1}\right)(x) \text {. }
$$

Then $S$ is an injective homomorphism. It follows from the relations that $S\left(e_{i}\right)=e_{i+1}$ and $S\left(g_{i}\right)=g_{i+1}$ for $i \leq n-1$. The map $S$ is called the shift endomorphism.

(3) The assignment $e_{i} \mapsto e_{n-i}, g_{i} \mapsto g_{n-i}$ determines an automorphism $\varrho_{n}$ of $W_{n, R}$, as the relations are preserved by this assignment. The shift map $S: W_{n, R} \rightarrow W_{n+1, R}$ satisfies $S=\varrho_{n+1} \circ \varrho_{n}$.

(4) The assignment $g_{i} \mapsto g_{i}$ and $e_{i} \mapsto e_{i}$ determines an anti-automorphism $\alpha$ of $W_{n, R}$.

(5) The assignment $g_{i} \mapsto g_{i}^{-1}, e_{i} \mapsto e_{i}$ determines an isomorphism $\beta$ from $W_{n, R}(\lambda, z, \delta)$ to $W_{n, R}\left(\lambda^{-1},-z, \delta\right)$.

We have the following relation of the BMW algebras to the Hecke algebras of type $A$ :

Proposition 3.4. Let $J_{n, R}$ denote the ideal $W_{n, R} e_{n-1} W_{n, R}$ in $W_{n, R}$.

(1) $J_{n, R}$ is the ideal in $W_{n, R}$ generated by $\left\{e_{1}, \ldots, e_{n-1}\right\}$.

(2) $W_{n, R} / J_{n, R}$ is isomorphic to the Hecke algebra over $\Lambda$ with generators $g_{i}^{ \pm 1}(1 \leq i \leq n-1)$ satisfying the braid relations and the quadratic relation $g_{i}^{2}=1-z g_{i}$.

Proof. For (1), it follows from the relation $e_{j} e_{j+1} e_{j}=e_{j}$ that all the $e_{i}$ 's are elements of $W_{n} e_{n-1} W_{n}$. Statement (2) is evident, since the $e_{i}$ 's are zero in the quotient.

REMARK 3.5. For $\omega \in \mathfrak{S}_{n}$, let $\omega=s_{i_{1}} \cdots s_{i_{r}}$ be a reduced expression for $\omega$ (in terms of the generators $s_{i}$ of the symmetric group). Let $g_{\omega}$ be the corresponding word in the generators $g_{i}$ of $W_{n, R}$. It follows from the proposition and well known facts concerning the Hecke algebra that $g_{\omega}$ is well defined modulo $J_{n, R}$. In fact, $g_{\omega}$ is well defined in $W_{n, R}$, not merely well defined modulo the ideal $J_{n, R}$; see Proposition 5.14.

Recall that $\Lambda$ denotes the ring

$$
\Lambda=\mathbb{Z}\left[\boldsymbol{\lambda}^{ \pm 1}, \boldsymbol{z}, \boldsymbol{\delta}^{ \pm 1}\right] /\left\langle\boldsymbol{\lambda}^{-1}-\boldsymbol{\lambda}=\boldsymbol{z}(\boldsymbol{\delta}-1)\right\rangle,
$$

where $\boldsymbol{\lambda}, \boldsymbol{z}, \boldsymbol{\delta}$ are indeterminates. The generic Birman-Wenzl-Murakami algebra is the BMW algebra $W_{n, \Lambda}$ over the ring $\Lambda$. Given a ring $R$ as above, we have two possible specializations to an algebra over $R$, namely $W_{n, R}$ 
and $W_{n, \Lambda} \otimes_{\Lambda} R$. We will show later that these are in fact isomorphic; see Corollary 5.43 .

3.2. The affine Birman-Wenzl-Murakami algebra. Let $S$ be a ring with distinguished elements $\lambda, z$, and $\delta$ as above, and with additional elements $q_{1}, q_{2}, \ldots$.

Definition 3.6. The affine Birman-Wenzl-Murakami algebra $\widehat{W}_{n, S}$ is the $S$-algebra with generators $x_{1}^{ \pm 1}, g_{i}^{ \pm 1}$ and $e_{i}(1 \leq i \leq n-1)$ and relations:

(1) (Inverses) $g_{i} g_{i}^{-1}=g_{i}^{-1} g_{i}=1$ and $x_{1} x_{1}^{-1}=x_{1}^{-1} x_{1}=1$.

(2) (Idempotent relation) $e_{i}^{2}=\delta e_{i}$.

(3) (Affine braid relations)

(a) $g_{i} g_{i+1} g_{i}=g_{i+1} g_{i} g_{i+1}$ and $g_{i} g_{j}=g_{j} g_{i}$ if $|i-j| \geq 2$.

(b) $x_{1} g_{1} x_{1} g_{1}=g_{1} x_{1} g_{1} x_{1}$ and $x_{1} g_{j}=g_{j} x_{1}$ if $j \geq 2$.

(4) (Commutation relations)

(a) $g_{i} e_{j}=e_{j} g_{i}$ and $e_{i} e_{j}=e_{j} e_{i}$ if $|i-j| \geq 2$.

(b) $x_{1} e_{j}=e_{j} x_{1}$ if $j \geq 2$.

(5) (Affine tangle relations)

(a) $e_{i} e_{i \pm 1} e_{i}=e_{i}$.

(b) $g_{i} g_{i \pm 1} e_{i}=e_{i \pm 1} e_{i}$ and $e_{i} g_{i \pm 1} g_{i}=e_{i} e_{i \pm 1}$.

(c) For $r \geq 1, e_{1} x_{1}^{r} e_{1}=q_{r} e_{1}$.

(6) (Kauffman skein relation) $g_{i}-g_{i}^{-1}=z\left(e_{i}-1\right)$.

(7) (Untwisting relations) $g_{i} e_{i}=e_{i} g_{i}=\lambda^{-1} e_{i}$ and $e_{i} g_{i \pm 1} e_{i}=\lambda e_{i}$.

(8) (Unwrapping relations) $e_{1} x_{1} g_{1} x_{1}=\lambda^{-1} e_{1}=x_{1} g_{1} x_{1} e_{1}$.

REMARK 3.7. Let $R$ be a ring with distinguished elements $\lambda, z$, and $\delta$ as above, and $S \supseteq R$ a ring with additional elements $q_{1}, q_{2}, \ldots$

(1) The assignment $e_{i} \mapsto e_{i}, g_{i} \mapsto g_{i}, x_{1} \mapsto x_{1}$ defines a homomorphism

$$
\iota: \widehat{W}_{n, S} \rightarrow \widehat{W}_{n+1, S}
$$

since the relations are preserved. It is not evident that $\iota$ is injective, but this will follow from the isomorphism $\widehat{W}_{n, S} \cong \widehat{\mathrm{KT}}_{n, S}$; see Corollary 6.15.

(2) The assignment $e_{i} \mapsto e_{i}, g_{i} \mapsto g_{i}$ defines an $R$-algebra homomorphism

$$
i_{n}: W_{n, R} \rightarrow \widehat{W}_{n, S}
$$

This map is always injective, as we will verify later. (For the moment we verify that $i_{n}: W_{n, R} \rightarrow \widehat{W}_{n, \widehat{R}}$ is injective. In fact, the assignment $e_{i} \mapsto e_{i}, g_{i} \mapsto g_{i}, x_{1} \mapsto 1, \boldsymbol{q}_{i} \mapsto \delta$ defines an $R$-algebra homomorphism from $f_{n}: \widehat{W}_{n, \widehat{R}} \rightarrow W_{n, R}$, and $f_{n} \circ i_{n}$ is the identity on $W_{n, R}$.) 
Note that the following diagram commutes:

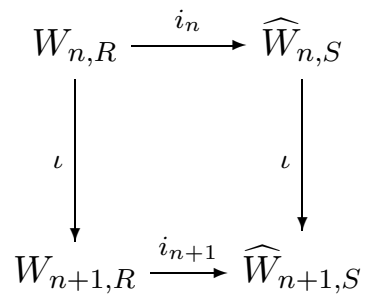

(3) The assignment $g_{i} \mapsto g_{i}, e_{i} \mapsto e_{i}, x_{1} \mapsto x_{1}$ determines an antiautomorphism $\alpha$ of $\widehat{W}_{n, S}$. One has $\alpha \circ i_{n}=i_{n} \circ \alpha$, where $i_{n}$ : $W_{n, R} \rightarrow \widehat{W}_{n, S}$ and $\alpha$ also denotes the anti-automorphism of $W_{n, R}$ of Remark 3.3.

(4) For $r \geq 1$, let $q_{-r}=f_{r}\left(q_{1}, \ldots, q_{r}\right)$, where $f_{r}$ is the polynomial of equation (2.1). The assignment $g_{i} \mapsto g_{i}^{-1}, e_{i} \mapsto e_{i}, x_{1} \mapsto x_{1}^{-1}$ determines an isomorphism

$$
\beta: \widehat{W}_{n, S}\left(\lambda, z, \delta,, q_{1}, q_{2}, \ldots\right) \rightarrow \widehat{W}_{n, S}\left(\lambda^{-1},-z, \delta, q_{-1}, q_{-2}, \ldots\right) .
$$

The following diagram commutes:

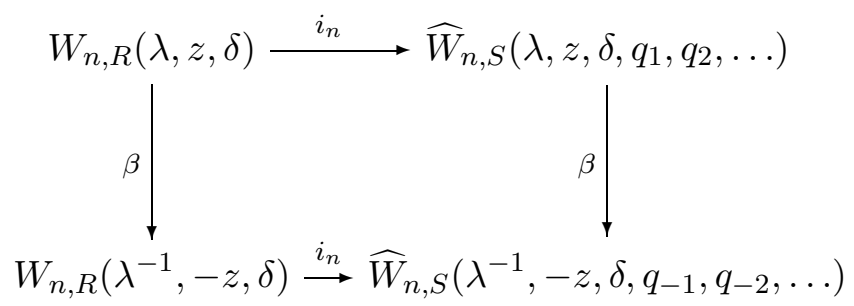

Lemma 3.8. The Kauffman skein relation implies

$$
g_{i}^{2}=1+\lambda^{-1} z e_{i}-z g_{i}, \quad g_{i}^{-2}=1-\lambda z e_{i}+z g_{i} .
$$

Proof. Multiply by $g_{i}$ or $g_{i}^{-1}$ and simplify using the untwisting relation.

LEMMA 3.9.

(1) $g_{i}^{ \pm 1}\left(g_{i+1} g_{i}\right)=\left(g_{i+1} g_{i}\right) g_{i+1}^{ \pm 1}$.

(2) $e_{i}\left(g_{i+1} g_{i}\right)=\left(g_{i+1} g_{i}\right) e_{i+1}$.

(3) $g_{i} g_{i+1} e_{i} e_{i+2}=g_{i+2} g_{i+1} e_{i} e_{i+2}$.

(4) If $i<m$, then

$$
\begin{aligned}
g_{i}^{ \pm 1}\left(g_{m} g_{m-1} \cdots g_{1}\right) & =\left(g_{m} g_{m-1} \cdots g_{1}\right) g_{i+1}^{ \pm 1}, \\
g_{i+1}^{ \pm 1}\left(g_{1} g_{2} \cdots g_{m}\right) & =\left(g_{1} g_{2} \cdots g_{m}\right) g_{i}^{ \pm 1} .
\end{aligned}
$$

(5) If $i<m$, then

$$
\begin{aligned}
e_{i}\left(g_{m} g_{m-1} \cdots g_{1}\right) & =\left(g_{m} g_{m-1} \cdots g_{1}\right) e_{i+1}, \\
e_{i+1}\left(g_{1} g_{2} \cdots g_{m}\right) & =\left(g_{1} g_{2} \cdots g_{m}\right) e_{i} .
\end{aligned}
$$


Proof. The first statement is just the braid relation. The second results from two applications of the tangle relation (5b) from Definition 3.6:

$$
e_{i}\left(g_{i+1} g_{i}\right)=e_{i} e_{i+1}=\left(g_{i+1} g_{i}\right) e_{i+1} .
$$

Statement (3) also results from two applications of the tangle relation (5b) from Definition 3.6:

$$
g_{i} g_{i+1} e_{i} e_{i+2}=e_{i+1} e_{i} e_{i+2}=e_{i+1} e_{i+2} e_{i}=g_{i+2} g_{i+1} e_{i+2} e_{i} .
$$

The first parts of statements (4) and (5) follow from statements (1) and (2) and the commutation relations (3a) and (4a) of Definition 3.6. The second parts of statements (4) and (5) follow by applying the anti-automorphism $\alpha$.

3.3. The elements $x_{i}$. For $2 \leq r \leq n$ define

$$
x_{r}=\left(g_{r-1} \cdots g_{1}\right) x_{1}\left(g_{1} \cdots g_{r-1}\right) .
$$

Proposition 3.10.

(1) For all $r$ and $j \notin\{r, r-1\}, g_{j} x_{r}=x_{r} g_{j}$.

(2) For all $r$ and $j \notin\{r, r-1\}, e_{j} x_{r}=x_{r} e_{j}$.

(3) For all $r$ and $j, x_{j} x_{r}=x_{r} x_{j}$.

Proof. For $j<r-1$, relations (1) and (2) follow by applying Lemma $3.9(4,5)$ and Definition 3.6(3b, 4b).

For $j>r$, relations (1) and (2) follow from Definition 3.6(3, 4).

For part (3), we first observe that $x_{1}$ commutes with $x_{r}$ for $r \geq 2$, by the braid relations Definition 3.6(3b). Namely,

$$
\begin{aligned}
x_{1} x_{r} & =x_{1}\left(g_{r-1} \cdots g_{2} g_{1}\right) x_{1}\left(g_{1} g_{2} \cdots g_{r-1}\right)=\left(g_{r-1} \cdots g_{2}\right) x_{1} g_{1} x_{1} g_{1}\left(g_{2} \cdots g_{r-1}\right) \\
& =\left(g_{r-1} \cdots g_{2}\right) g_{1} x_{1} g_{1} x_{1}\left(g_{2} \cdots g_{r-1}\right)=\left(g_{r-1} \cdots g_{2} g_{1}\right) x_{1}\left(g_{1} g_{2} \cdots g_{r-1}\right) x_{1} \\
& =x_{r} x_{1} .
\end{aligned}
$$

Finally, if $j<r$, then $x_{j} x_{r}=\left(g_{j-1} \cdots g_{1}\right) x_{1}\left(g_{1} \cdots g_{j-1}\right) x_{r}=x_{r}\left(g_{j-1} \cdots g_{1}\right) x_{1}\left(g_{1} \cdots g_{j-1}\right)=x_{r} x_{j}$, since $x_{r}$ commutes with $g_{i}$ for $i \leq j-1$ and with $x_{1}$.

Proposition 3.11.

(1) For $r<n, g_{r} x_{r}=x_{r+1} g_{r}^{-1}$ and $g_{r}^{-1} x_{r+1}=x_{r} g_{r}$.

(2) For $r<n$,

$$
\begin{aligned}
g_{r} x_{r+1} & =x_{r} g_{r}-z x_{r+1}+z \lambda e_{r} x_{r+1}, \\
g_{r}^{-1} x_{r} & =x_{r+1} g_{r}^{-1}+z x_{r}-z e_{r} x_{r} .
\end{aligned}
$$

(3) For $r<n, e_{r} x_{r}=\lambda^{-2} e_{r} x_{r+1}^{-1}$ and $x_{r} e_{r}=\lambda^{-2} x_{r+1}^{-1} e_{r}$.

(4) For $r<n, e_{r} x_{r}^{-1}=\lambda^{2} e_{r} x_{r+1}$ and $x_{r}^{-1} e_{r}=\lambda^{2} x_{r+1} e_{r}$. 
Proof. Statement (1) follows from the definition of $x_{r}$. Statement (2) uses the Kauffman skein relation, the quadratic relation of Lemma 3.8, and part (1). Statement (3) is equivalent to

$$
e_{r} x_{r} x_{r+1}=\lambda^{-2} e_{r} .
$$

For $r=1$, this follows from Definition 3.6(7, 8). The proof is completed by induction on $r$. For $r \geq 2$, one has (using braid and tangle relations)

$$
\begin{aligned}
e_{r} x_{r} x_{r+1}= & e_{r}\left(g_{r-1} \cdots g_{1}\right) x_{1}\left(g_{1} \cdots g_{r-2}\left(g_{r-1} g_{r} g_{r-1}\right) g_{r-2} \cdots g_{1}\right) x_{1}\left(g_{1} \cdots g_{r}\right) \\
= & e_{r}\left(g_{r-1} \cdots g_{1}\right) x_{1}\left(g_{1} \cdots g_{r-2}\left(g_{r} g_{r-1} g_{r}\right) g_{r-2} \cdots g_{1}\right) x_{1}\left(g_{1} \cdots g_{r}\right) \\
= & \left(e_{r} g_{r-1} g_{r}\right)\left(g_{r-2} \cdots g_{1}\right) x_{1}\left(g_{1} \cdots g_{r-2} g_{r-1} g_{r-2} \cdots g_{1}\right) \\
& \cdot x_{1}\left(g_{1} \cdots g_{r-2}\right)\left(g_{r} g_{r-1} g_{r}\right) \\
= & \left(e_{r} e_{r-1}\right)\left(g_{r-2} \cdots g_{1}\right) x_{1}\left(g_{1} \cdots g_{r-2} g_{r-1} g_{r-2} \cdots g_{1}\right) \\
& \cdot x_{1}\left(g_{1} \cdots g_{r-2}\right)\left(g_{r-1} g_{r} g_{r-1}\right) \\
= & e_{r} e_{r-1} x_{r-1} x_{r} g_{r} g_{r-1} \\
= & \lambda^{-2} e_{r} e_{r-1} g_{r} g_{r-1} \quad \text { by the induction assumption } \\
= & \lambda^{-2} e_{r} e_{r-1} e_{r}=\lambda^{-2} e_{r} .
\end{aligned}
$$

Statement (4) follows from (3) by applying the isomorphism $\beta$ of Remark 3.7 .

The next proposition contains general unwrapping and affine braid relations:

Proposition 3.12. For all $n \geq 1$ and all $r \geq 1$,

(1) $g_{n} x_{n} g_{n} x_{n}=x_{n} g_{n} x_{n} g_{n}$.

(2) $e_{n} x_{n} g_{n} x_{n}=\lambda^{-1} e_{n}$.

(3) $e_{n} x_{n}^{r} g_{n} x_{n}=\lambda^{-2} e_{n} x_{n}^{r-1} g_{n}^{-1}$.

(4) $e_{n} x_{n}^{-r} g_{n}^{-1} x_{n}^{-1}=\lambda^{2} e_{n} x_{n}^{-r+1} g_{n}$.

Proof. Statement (1) is equivalent to

$$
x_{n+1} x_{n}=x_{n} x_{n+1},
$$

so follows from Proposition 3.10. Statement (2) follows from (3) and the untwisting relation of Definition 3.6. For statement (3), we have

$$
\begin{aligned}
e_{n} x_{n}^{r} g_{n} x_{n} & =\lambda^{-2} e_{n} x_{n}^{r-1} x_{n+1}^{-1} g_{n} x_{n} \\
& =\lambda^{-2} e_{n} x_{n}^{r-1} g_{n}^{-1} x_{n}^{-1} x_{n}=\lambda^{-2} e_{n} x_{n}^{r-1} g_{n}^{-1} .
\end{aligned}
$$

by Proposition 3.11. Statement (4) follows by applying the isomorphism $\beta$ to $(3)$.

Corollary 3.13. For $r \geq 1, e_{1} x_{1}^{-r} e_{1}=f_{r}\left(q_{1}, \ldots, q_{r}\right) e_{1}$, where $f_{r}$ is the polynomial of equation (2.1) in Section 2.4. 
Proof. For $r \geq 1$, set $\psi_{r}=e_{1} x_{1}^{r} e_{1}=q_{r} e_{1}$, and $\psi_{-r}=e_{1} x_{1}^{-r} e_{1}$. For $a \geq 1$ and $b \geq 0$, define $\psi_{a, b}=e_{1} x_{1}^{-b} g_{1} x_{1}^{a} e_{1}$, and $\psi_{a, b}^{-}=e_{1} x_{1}^{-b} g_{1}^{-1} x_{1}^{a} e_{1}$. In particular, $\psi_{a, 0}=e_{1} g_{1} x_{1}^{a} e_{1}=\lambda^{-1} q_{a} e_{1}$. We have

$$
\psi_{-r}=e_{1} x_{1}^{-r} e_{1}=\lambda^{-1} e_{1} x_{1}^{-r} g_{1}^{-1} e_{1}=\lambda \psi_{1, r-1},
$$

and

$$
\psi_{a, b}^{-}=\lambda^{2} \psi_{a+1, b-1},
$$

using Proposition 3.12(4). Moreover, for $a, b \geq 1$,

$$
\begin{aligned}
\psi_{a, b} & =e_{1} x_{1}^{-b} g_{1} x_{1}^{a} e_{1} \\
& =e_{1} x_{1}^{-b} g_{1}^{-1} x_{1}^{a} e_{1}+z\left(e_{1} x_{1}^{-b} e_{1} x_{1}^{a} e_{1}-e_{1} x_{1}^{a-b} e_{1}\right) \\
& =\psi_{a, b}^{-}+z\left(\psi_{-b} q_{a}-\psi_{a-b}\right) \\
& =\psi_{a+1, b-1}+z\left(\psi_{-b} q_{a}-\psi_{a-b}\right),
\end{aligned}
$$

by the Kauffman skein relation, and equation 3.2. Comparing the recursion relations of equations 3.1 and 3.3 with those of Lemma 2.8 gives that $\psi_{-r}=$ $f_{r}\left(q_{1}, \ldots, q_{r}\right) e_{1}$.

3.4. $\widehat{W}_{n}$ as a $\widehat{W}_{n-1}$-bimodule. $W_{n, R}$ has a simple structure as a

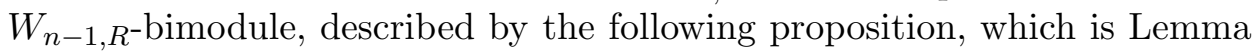
3.1 from [2].

Proposition 3.14. $W_{n, R}$ is the span of elements of the form $a \chi b$, where $a, b \in W_{n-1, R}$ and $\chi \in\left\{g_{n-1}^{ \pm 1}, e_{n-1}, 1\right\}$.

We generalize this result to the affine Birman-Wenzl-Murakami algebras in this section. Fix $S$ and let $\widehat{W}_{n}$ denote $\widehat{W}_{n, S}$.

LEMma 3.15 .

(1) For $n \geq 1$ and $s \in \mathbb{Z}$, there exists an element $b \in \widehat{W}_{n-1}$ such that

$$
e_{n} x_{n}^{s} e_{n}=b e_{n} .
$$

(2) For $n \geq 2$, let a be an element of $\widehat{W}_{n}$ of the form

$$
a=z_{0} x_{n-1}^{r_{1}} z_{1} x_{n-1}^{r_{2}} \cdots z_{k-1} x_{n-1}^{r_{k}} z_{k},
$$

where $z_{i} \in\left\{e_{n-1}, g_{n-1}\right\}$ for all $i$, and $r_{i} \geq 1$. There exists $b \in \widehat{W}_{n-1}$ such that

$$
e_{n} a e_{n}=b e_{n} .
$$

Proof. For $n=1$, the first assertion follows from the relation (5c) of Definition 3.6 and Corollary 3.13. We take this as the base for an inductive proof of both assertions. 
For $n \geq 2$ and $s \geq 1$, the first assertion follows from the second, since $x_{n}^{s}=\left(g_{n-1} x_{n-1} g_{n-1}\right)^{s}$. The assertion for $s \leq-1$ follows by applying the symmetry $\beta$.

The proof of statement (2) is by induction first on $n$ and then on the number $k$ of factors $x_{n-1}^{r_{j}} z_{j}$ in the expression for $a$. For $n \geq 2$ and $k=0$, the statement is immediate from the BMW relations. Fix $n \geq 2, k \geq 1$, and $a$ of the form above with $k$ factors; assume inductively that

(1) $e_{m} x_{m}^{s} e_{m} \in \widehat{W}_{m-1} e_{m}$ for $m<n$ and $s \geq 1$, and

(2) $e_{n} a^{\prime} e_{n} \in \widehat{W}_{n-1} e_{n}$ if $a^{\prime}$ is of the same form with fewer than $k$ factors.

We consider several cases:

(a) Two successive $z_{i}$ are equal to $e_{n-1}$. Then

$$
a=a^{\prime} e_{n-1} x_{n-1}^{s} e_{n-1} a^{\prime \prime}=a^{\prime} b e_{n-1} a^{\prime \prime},
$$

where $b \in \widehat{W}_{n-2}$, by the induction hypothesis. Thus

$$
e_{n} a e_{n}=b e_{n} a^{\prime} e_{n-1} a^{\prime \prime} e_{n} \in \widehat{W}_{n-1} e_{n},
$$

since $a^{\prime} e_{n-1} a^{\prime \prime}$ has fewer than $k$ factors.

(b) For some $i, z_{i}=e_{n-1}$ and $z_{i+1}=g_{n-1}$. Then

$$
\begin{aligned}
e_{n} a e_{n} & =e_{n} a^{\prime} e_{n-1} x_{n-1}^{r} g_{n-1} a^{\prime \prime} e_{n} \\
& =\lambda^{-2} e_{n} a^{\prime} e_{n-1} x_{n-1}^{r-1} g_{n-1}^{-1} x_{n-1}^{-1} a^{\prime \prime} e_{n} \quad \text { (by Proposition 3.12) } \\
& =\lambda^{-2} e_{n} a^{\prime} e_{n-1} x_{n-1}^{r-1}\left[g_{n-1}+z e_{n-1}-z\right] x_{n-1}^{-1} a^{\prime \prime} e_{n} \\
& \equiv \lambda^{-2} e_{n} a^{\prime} e_{n-1} x_{n-1}^{r-1} g_{n-1} x_{n-1}^{-1} a^{\prime \prime} e_{n} \bmod \widehat{W}_{n-1} e_{n},
\end{aligned}
$$

using case (a) and the induction hypothesis. If $a^{\prime \prime}=1$, then the final expression is equal to

$$
\lambda^{-2} e_{n} a^{\prime} e_{n-1} x_{n-1}^{r-1} g_{n-1} e_{n} x_{n-1}^{-1} .
$$

Repeating this step $r$ times in all, we get

$$
\begin{aligned}
e_{n} a e_{n} & \equiv \lambda^{-2 r} e_{n} a^{\prime} e_{n-1} g_{n-1} e_{n} x_{n-1}^{-r} \bmod \widehat{W}_{n-1} e_{n} \\
& =\lambda^{-2 r-1} e_{n} a^{\prime} e_{n-1} e_{n} x_{n-1}^{-r} .
\end{aligned}
$$

By the induction hypothesis, this is in $\widehat{W}_{n-1} e_{n}$. If $a^{\prime \prime} \neq 1$, then $a^{\prime \prime}=$

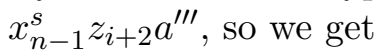

$$
e_{n} a e_{n} \equiv \lambda^{-2} e_{n} a^{\prime} e_{n-1} x_{n-1}^{r-1} g_{n-1} x_{n-1}^{s-1} z_{i+2} a^{\prime \prime \prime} e_{n} \bmod \widehat{W}_{n-1} e_{n} .
$$

If $r>s$, we repeat this step $s$ times in all, obtaining finally

$$
e_{n} a e_{n} \equiv \lambda^{-2 s} e_{n} a^{\prime} e_{n-1} x_{n-1}^{r-s} g_{n-1} z_{i+2} a^{\prime \prime \prime} e_{n} \bmod \widehat{W}_{n-1} e_{n} .
$$


Now $g_{n-1} z_{i+2}$ is a linear combination of $e_{n-1}, g_{n-1}$, and 1 , so by the induction hypothesis, the latter expression is in $\widehat{W}_{n-1} e_{n}$. The cases $r<s$ and $r=s$ are similar.

(c) For some $i, z_{i}=g_{n-1}$ and $z_{i+1}=e_{n-1}$. This is essentially the same as case (b).

(d) For all $i, z_{i}=g_{n-1}$. If $a=g_{n-1} x_{n-1}^{r} g_{n-1}$, then

$$
\begin{aligned}
e_{n} a e_{n} & =e_{n} g_{n-1} x_{n-1}^{r} g_{n-1} e_{n} \\
& \equiv e_{n} g_{n-1} x_{n-1}^{r} g_{n-1}^{-1} e_{n} \bmod \widehat{W}_{n-1} e_{n} \quad(\text { as in case (b)) } \\
& =e_{n} g_{n-1} g_{n} x_{n-1}^{r} g_{n}^{-1} g_{n-1}^{-1} e_{n} \\
& =e_{n} e_{n-1} x_{n-1}^{r} e_{n-1} e_{n} \in \widehat{W}_{n-1} e_{n},
\end{aligned}
$$

by case (a). Otherwise, $a=g_{n-1} x_{n-1}^{r} g_{n-1} x_{n-1}^{s} g_{n-1} a^{\prime}$, and

$$
\begin{aligned}
e_{n} a e_{n} & =e_{n} g_{n-1} x_{n-1}^{r} g_{n-1} x_{n-1}^{s} g_{n-1} a^{\prime} e_{n} \\
& \equiv e_{n} g_{n-1}^{-1} x_{n-1}^{r} g_{n-1} x_{n-1}^{s} g_{n-1} a^{\prime} e_{n} \bmod \widehat{W}_{n-1} e_{n} \quad \text { (as in case (b)). }
\end{aligned}
$$

The affine braid relation of Proposition 3.12 implies

$$
x_{n-1}^{r} g_{n-1} x_{n-1} g_{n-1}=g_{n-1} x_{n-1} g_{n-1} x_{n-1}^{r},
$$

or

$$
g_{n-1}^{-1} x_{n-1}^{r} g_{n-1} x_{n-1}=x_{n-1} g_{n-1} x_{n-1}^{r} g_{n-1}^{-1} .
$$

Applying this, we get

$$
e_{n} a e_{n} \equiv e_{n} x_{n-1} g_{n-1} x_{n-1}^{r} g_{n-1}^{-1} x_{n-1}^{s-1} g_{n-1} a^{\prime} e_{n} \bmod \widehat{W}_{n-1} e_{n} .
$$

Now we can change the $g_{n-1}^{-1}$ to $g_{n-1}$ while maintaining congruence mod $\widehat{W}_{n-1} e_{n}$, as in case (b), so

$$
e_{n} a e_{n} \equiv x_{n-1} e_{n} g_{n-1} x_{n-1}^{r} g_{n-1} x_{n-1}^{s-1} g_{n-1} a^{\prime} e_{n} \bmod \widehat{W}_{n-1} e_{n} .
$$

Repeating this step a total of $s$ times, we get

$$
e_{n} a e_{n} \equiv x_{n-1}^{s} e_{n} g_{n-1} x_{n-1}^{r} g_{n-1}^{2} a^{\prime} e_{n} \bmod \widehat{W}_{n-1} e_{n} .
$$

Since $g_{n-1}^{2}$ is a linear combination of $e_{n-1}, g_{n-1}$, and 1 , the last expression is in $\widehat{W}_{n-1} e_{n}$ by the induction assumption.

For the remainder of this section, we maintain the following notation: $A_{1}$ denotes the linear span of $x_{1}^{r}$ for $r \in \mathbb{Z}$. (Thus $A_{1}=\widehat{W}_{1}$.) For each $n \geq 2$, $A_{n}$ denotes the linear span of $e_{n-1}, g_{n-1}^{ \pm 1}$, and $x_{n}^{r}$ for $r \in \mathbb{Z}$.

The following proposition is the analogue for the $\widehat{W}_{n}$ of Lemma 3.1 in [2]. This result is due to Häring-Oldenburg [5].

Proposition 3.16. Every element of $\widehat{W}_{n}$ is a linear combination of elements of the form $a \chi b$, where $a, b \in \widehat{W}_{n-1}$ and $\chi \in\left\{e_{n-1}, g_{n-1}^{ \pm 1}\right\} \cup\left\{x_{n}^{r}: r \in \mathbb{Z}\right\}$. 
Proof. We have to show that for all $n \geq 1, \widehat{W}_{n}=\widehat{W}_{n-1} A_{n} \widehat{W}_{n-1}$. Since $\widehat{W}_{n}$ is generated as an algebra by $\widehat{W}_{n-1}$ and $A_{n}$, it suffices to show that $A_{n} \widehat{W}_{n-1} A_{n} \subseteq \widehat{W}_{n-1} A_{n} \widehat{W}_{n-1}$.

The assertion is evident for $n=1$. We assume it holds for a particular $n \geq 1$ and prove that $A_{n+1} \widehat{W}_{n} A_{n+1} \subseteq \widehat{W}_{n} A_{n+1} \widehat{W}_{n}$. By the induction assumption, $\widehat{W}_{n}=\widehat{W}_{n-1} A_{n} \widehat{W}_{n-1}$, so $A_{n+1} \widehat{W}_{n} A_{n+1}=A_{n+1} \widehat{W}_{n-1} A_{n} \widehat{W}_{n-1} A_{n+1}$ $=\widehat{W}_{n-1} A_{n+1} A_{n} A_{n+1} \widehat{W}_{n-1}$. Thus it suffices to show that

$$
A_{n+1} A_{n} A_{n+1} \subseteq \widehat{W}_{n} A_{n+1} \widehat{W}_{n} .
$$

We consider several cases:

(a) $\chi_{n}^{\prime} \chi_{n-1} \chi_{n}^{\prime \prime} \in \widehat{W}_{n} A_{n+1} \widehat{W}_{n}$, where $\chi_{n}^{\prime}, \chi_{n}^{\prime \prime} \in\left\{e_{n}, g_{n}^{ \pm 1}\right\}$ and $\chi_{n-1} \in$ $\left\{e_{n-1}, g_{n-1}^{ \pm 1}\right\}$. This follows easily from the BMW relations.

(b) $e_{n} x_{n}^{r} e_{n} \in \widehat{W}_{n-1} e_{n} \subseteq \widehat{W}_{n} A_{n+1}$. This follows from Lemma 3.15 .

(c) $g_{n}^{ \pm 1} x_{n}^{r} e_{n} \in \widehat{W}_{n} e_{n} \subseteq \widehat{W}_{n} A_{n+1}$, and $e_{n} x_{n}^{r} g_{n}^{ \pm 1} \in e_{n} \widehat{W}_{n} \subseteq A_{n+1} \widehat{W}_{n}$. The second statement follows from the first by applying the symmetry $\alpha$. Note that

$$
g_{n}^{-1} x_{n}^{r} e_{n} \equiv g_{n} x_{n}^{r} e_{n} \bmod \widehat{W}_{n} e_{n},
$$

by the Kauffman skein relation and case (b). Moreover, the assertion for $r \leq-1$ follows from the assertion for $r \geq 1$ by applying the symmetry $\beta$. For $r \geq 1$, we have

$$
g_{n} x_{n}^{r} e_{n}=\lambda^{-2} x_{n}^{-1} g_{n}^{-1} x_{n}^{r-1} e_{n} \equiv x_{n}^{-1} g_{n} x_{n}^{r-1} e_{n} \bmod \widehat{W}_{n} e_{n},
$$

by the unwrapping relation of Proposition 3.12. Repeating this step a total of $r$ times gives

$$
\begin{aligned}
g_{n} x_{n}^{r} e_{n} & \equiv \lambda^{-2 r} x_{n}^{-r} g_{n} e_{n} \bmod \widehat{W}_{n} e_{n} \\
& =\lambda^{-2 r-1} x_{n}^{-r} e_{n} .
\end{aligned}
$$

(d) $A_{n+1} A_{n} x_{n+1}^{t} \subseteq \widehat{W}_{n} A_{n+1} \widehat{W}_{n}$ and $x_{n+1}^{t} A_{n} A_{n+1} \subseteq \widehat{W}_{n} A_{n+1} \widehat{W}_{n}$. The second assertion follows from the first by applying the symmetry $\alpha$. Since $x_{n+1}^{t}$ commutes with $A_{n}$, it suffices to show $A_{n+1} x_{n+1}^{t} \subseteq \widehat{W}_{n} A_{n+1} \widehat{W}_{n}$. Since $x_{n+1}^{s} x_{n+1}^{t}=x_{n+1}^{s+t} \in \widehat{W}_{n} A_{n+1} \widehat{W}_{n}$, we only have to check that $e_{n} x_{n+1}^{t} \in$ $\widehat{W}_{n} A_{n+1} \widehat{W}_{n}$, and $g_{n}^{ \pm 1} x_{n+1}^{t} \in \widehat{W}_{n} A_{n+1} \widehat{W}_{n}$.

We have

$$
e_{n} x_{n+1}^{t}=\lambda^{-2 t} e_{n} x_{n}^{-t} \in \widehat{W}_{n} A_{n+1} \widehat{W}_{n},
$$

by Proposition 3.11. It follows from this and the Kauffman skein relation that

$$
g_{n} x_{n+1}^{t} \equiv g_{n}^{-1} x_{n+1}^{t} \bmod \widehat{W}_{n} A_{n+1} \widehat{W}_{n} .
$$

Moreover, for $t \geq 1$,

$$
g_{n}^{-1} x_{n+1}^{t}=x_{n} g_{n} x_{n+1}^{t-1} \equiv x_{n} g_{n}^{-1} x_{n+1}^{t-1} \bmod \widehat{W}_{n} A_{n+1} \widehat{W}_{n} .
$$


Repeating this step $t$ times gives

$$
g_{n}^{-1} x_{n+1}^{t} \equiv x_{n}^{t} g_{n}^{-1} \bmod \widehat{W}_{n} A_{n+1} \widehat{W}_{n} .
$$

Thus for $t \geq 1$,

$$
g_{n}^{ \pm 1} x_{n+1}^{t} \in \widehat{W}_{n} A_{n+1} \widehat{W}_{n},
$$

and the same statement for $t \leq-1$ follows by applying the symmetry $\beta$.

Proposition 3.17. For $n \geq 1, e_{n} \widehat{W}_{n} e_{n}=\widehat{W}_{n-1} e_{n}$.

Proof. We only have to prove the containment $e_{n} \widehat{W}_{n} e_{n} \subseteq \widehat{W}_{n-1} e_{n}$. This is obvious for $n=1$. For $n \geq 2$, we have

$$
e_{n} \widehat{W}_{n} e_{n}=e_{n} \widehat{W}_{n-1} A_{n} \widehat{W}_{n-1} e_{n}=\widehat{W}_{n-1} e_{n} A_{n} e_{n} \widehat{W}_{n-1} .
$$

But $e_{n} A_{n} e_{n} \subseteq \widehat{W}_{n-1} e_{n}$ by the BMW relations and Lemma 3.15 .

COROLlary 3.18. For $n \geq 1, e_{n} W_{n} e_{n}=W_{n-1} e_{n}$.

LEMmA 3.19. For $n \geq 1, g_{n}^{ \pm 1} \widehat{W}_{n} e_{n} \subseteq \widehat{W}_{n} e_{n}$ and $x_{n+1}^{r} \widehat{W}_{n} e_{n} \subseteq \widehat{W}_{n} e_{n}$.

Proof. Let $\chi$ be one of $x_{n+1}^{r}$ or $g_{n}^{ \pm 1}$. We have

$$
\chi \widehat{W}_{n} e_{n}=\chi \widehat{W}_{n-1} A_{n} \widehat{W}_{n-1} e_{n}=\widehat{W}_{n-1} \chi A_{n} e_{n} \widehat{W}_{n-1} .
$$

But $g_{n}^{ \pm 1} A_{n} e_{n} \subseteq \widehat{W}_{n} e_{n}$ by the BMW relations and case (c) in the proof of Proposition 3.16. Likewise, $x_{n+1}^{r} A_{n} e_{n}=A_{n} x_{n+1}^{r} e_{n}=\lambda^{-2 r} x_{n}^{-r} e_{n} \subseteq \widehat{W}_{n} e_{n}$. Thus $\chi \widehat{W}_{n} e_{n} \subseteq \widehat{W}_{n} e_{n} \widehat{W}_{n-1}=\widehat{W}_{n} e_{n}$.

Proposition 3.20. For $n \geq 1, \widehat{W}_{n+1} e_{n}=\widehat{W}_{n} e_{n}$.

Proof. We have $\widehat{W}_{n+1} e_{n}=\widehat{W}_{n} A_{n+1} \widehat{W}_{n} e_{n} \subseteq \widehat{W}_{n} e_{n}$, by Proposition 3.17 and Lemma 3.19.

Corollary 3.21. For $n \geq 1, W_{n+1} e_{n}=W_{n} e_{n}$.

Note that Corollaries 3.18 and 3.21 also follow directly from Proposition 3.14 by similar reasoning.

3.5. A homomorphism from $\widehat{W}_{n}$ to $\widehat{\mathrm{KT}}_{n}$. Let $R$ be a ring with distinguished elements $\lambda, z$, and $\delta$ as above, and $S \supseteq R$ a ring with additional elements $q_{1}, q_{2}, \ldots$.

Let $X_{1}, G_{i}$, and $E_{i}$ denote the affine tangle diagrams shown in the figure following Definition 2.5, regarded as elements of the affine Kauffman tangle algebra $\widehat{\mathrm{KT}}_{n, S}$.

Proposition 3.22. The assignment $x_{1} \mapsto X_{1}, g_{i} \mapsto G_{i}$, and $e_{i} \mapsto E_{i}$ determines an $S$-algebra homomorphism $\varphi$ from $\widehat{W}_{n, S}$ to $\widehat{\mathrm{KT}}_{n, S}$. 
Proof. One needs to check that $X_{1}, G_{i}$, and $E_{i}$ satisfy the relations of the affine BMW algebra (Definition 3.6), using regular isotopy of affine tangles and the relations of the affine Kauffman tangle algebra. The inverses of $G_{i}$ and $X_{1}$ in $\widehat{\mathrm{KT}}_{n, S}$ are represented by the tangle diagrams with the crossings reversed:

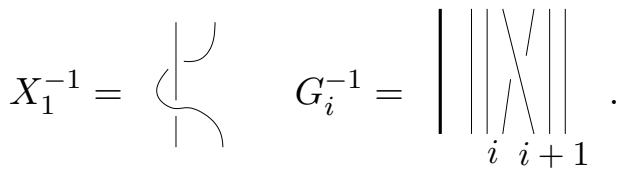

The verification that these elements are in fact inverses involves applications of Reidemeister II.

The Kauffman skein relation for $G_{i}$ depends on point (1) of Definition 2.6. The relation $E_{i}^{2}=\delta E_{i}$ results from point (3) of Definition 2.6. The relation $E_{1} X_{1}^{r} E_{1}=q_{r} E_{1}$ results from point (2) of Definition 2.9.

The affine braid relations result from applications of Reidemeister moves II and III. The remaining commutation relations and the tangle relation (6a) only use planar isotopy, while (6b) also uses Reidemeister move II. The untwisting relations (7) follow from the untwisting relation (2) for $\widehat{\mathrm{KT}}_{n, S}$.

Finally, the unwrapping relation follows from the following graphical "computation", which we give here for the sake of illustration. The first equality of pictures is just isotopy, the second a Reidemeister III move, the third an untwisting move, and the last two are Reidemeister II moves.

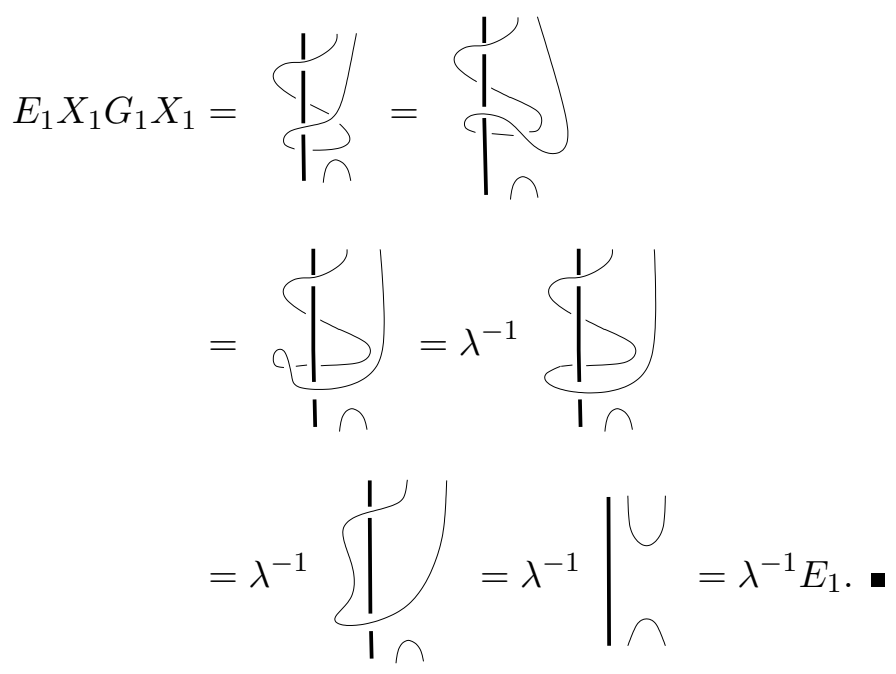

Proposition 3.23. The assignment $g_{i} \mapsto G_{i}$ and $e_{i} \mapsto E_{i}$ determines an R-algebra homomorphism $\varphi$ from $W_{n, R}$ to $\mathrm{KT}_{n, R}$.

Proof. One has to verify relations, as in the previous proof. 
REMARK 3.24.

(1) The homomorphism $\varphi: \widehat{W}_{n} \rightarrow \widehat{\mathrm{KT}}_{n}$ gives us a trace $\varepsilon: \widehat{W}_{n} \rightarrow S$ defined by $\varepsilon=\varepsilon \circ \varphi$.

(2) The following diagram commutes (see Propositions 3.22 and 3.23 and Remarks 2.11 and 3.7):

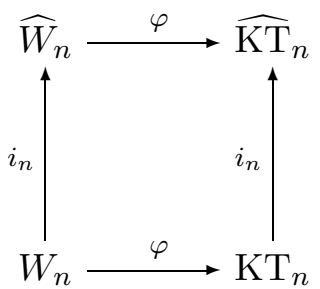

(3) The following diagrams commute:

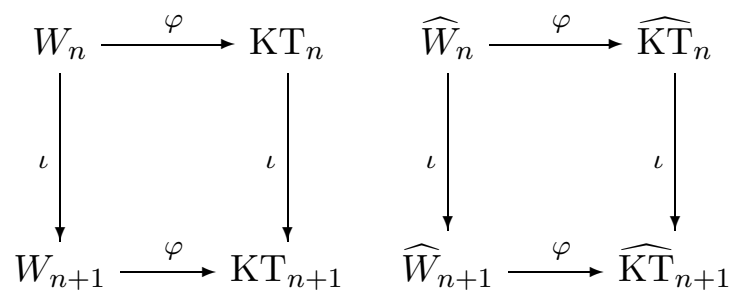

(4) The following diagrams commute (see Section 2.6, Remark 3.3, and Remark 3.7):
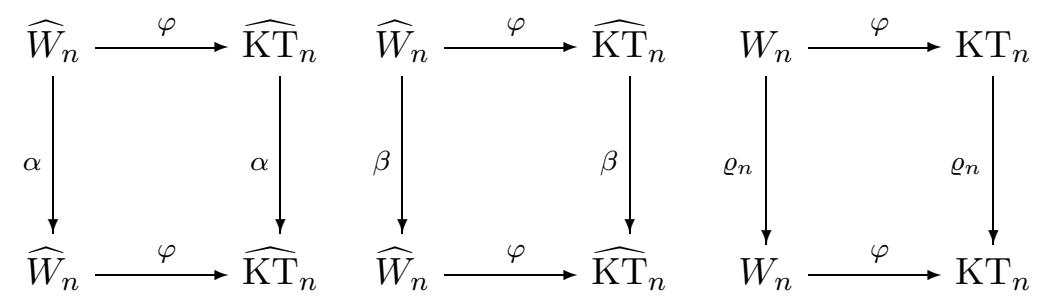

(5) One has $\varphi\left(x_{r}\right)=X_{r}$ for all $r$.

We will eventually show $\varphi: \widehat{W}_{n, S} \rightarrow \widehat{\mathrm{KT}}_{n, S}$ is an isomorphism for any $S$.

\section{THE AFFINE BRAUER ALGEBRA}

4.1. The Brauer algebra. The Brauer algebra $D_{n}$ is an algebra of planar $(n, n)$-tangle diagrams in which crossings are ignored. The precise definition follows.

Fix points $a_{i}$ in $I$, for $i \geq 0$, as in the description of $(n, n)$-tangles. For convenience write $\boldsymbol{i}=\left(a_{i}, 1\right)$ and $\overline{\boldsymbol{i}}=\left(a_{i}, 0\right)$.

Definition 4.1. An $(n, n)$-Brauer diagram (also called an $n$-connector) consists of a collection of $n$ curves in the rectangle $R=I \times I$ such that 
(1) The curves connect the points $\{\mathbf{1}, \ldots, \boldsymbol{n}, \overline{\mathbf{1}}, \ldots, \overline{\boldsymbol{n}}\}$ in pairs.

(2) For each curve $C$ in the collection, the intersection of $C$ with $\partial(R)$ consists of the two endpoints of $C$.

Consider the free $\mathbb{Z}\left[\boldsymbol{\delta}^{ \pm 1}\right]$-module $D_{n}$ with basis the set of $(n, n)$-Brauer diagrams. The product of two Brauer diagrams is defined to be a certain multiple of another Brauer diagram. Namely, given two Brauer diagrams $a, b$, first "stack" $b$ over $a$ (as for tangle diagrams). Let $r$ denote the number of closed curves in the interior of $R$ in the resulting planar "tangle", and let $c$ be the Brauer diagram obtained by removing all the closed curves. Then

$$
a b=\boldsymbol{\delta}^{r} c .
$$

Definition 4.2. The Brauer algebra $D_{n}$ over $\mathbb{Z}\left[\boldsymbol{\delta}^{ \pm 1}\right]$ is the free $\mathbb{Z}\left[\boldsymbol{\delta}^{ \pm 1}\right]$ module with basis the set of $(n, n)$-Brauer diagrams, with the bilinear product determined by the multiplication of Brauer diagrams.

The Brauer algebras were introduced by R. Brauer [3] as a device for studying the invariant theory of orthogonal and symplectic groups. The generic structure of the Brauer algebras, and conditions for semisimplicity of $D_{n} \otimes_{\mathbb{Z}\left[\boldsymbol{\delta}^{ \pm 1}\right]} k$, where $k$ is a field, were determined by H. Wenzl [20].

Note that the Brauer diagrams with only vertical strands, that is, diagrams in which upper points are paired only with lower points, are in bijection with permutations of $\{1, \ldots, n\}$, and that the multiplication of two such diagrams coincides with the multiplication of permutations. Thus the Brauer algebra contains the group algebra of the permutation group $\mathfrak{S}_{n}$ (over $\mathbb{Z}\left[\boldsymbol{\delta}^{ \pm 1}\right]$ ).

4.2. The affine Brauer algebra. We will define the affine Brauer algebra as a sort of "wreath product" of $\mathbb{Z}$ with the Brauer algebra (containing the wreath product of $\mathbb{Z}$ with $\mathfrak{S}_{n}$ ).

Definition 4.3. A colored $(n, n)$-Brauer diagram, or colored $n$-connector is a Brauer diagram in which each strand is labeled by an integer.

We will define the affine Brauer algebra over the ring

$$
\mathbb{Z}\left[\boldsymbol{\delta}^{ \pm 1}\right]^{\wedge}=\mathbb{Z}\left[\boldsymbol{\delta}^{ \pm 1}, \boldsymbol{q}_{1}, \boldsymbol{q}_{2}, \ldots\right],
$$

where $\boldsymbol{q}_{1}, \boldsymbol{q}_{2}, \ldots$ are indeterminates.

Order the points $\{\mathbf{1}, \ldots, \boldsymbol{n}, \overline{\mathbf{1}}, \ldots, \overline{\boldsymbol{n}}\}$ by $\mathbf{1}<\cdots<\boldsymbol{n}<\overline{\boldsymbol{n}}<\cdots<\overline{\mathbf{1}}$. The colors on the strands of a colored Brauer diagram should be regarded as assigning integer values to oriented strands of the Brauer diagram. If a strand is colored by the integer $r$, then the strand endowed with the positive orientation (i.e. the orientation from a lower numbered vertex to a higher numbered vertex) takes the value $r$, but the same strand endowed with the negative orientation takes the value $-r$. 
Consider the free $\mathbb{Z}\left[\boldsymbol{\delta}^{ \pm 1}\right]^{\wedge}$-module $\widehat{D}_{n}$ with basis the set of colored $(n, n)$ Brauer diagrams. The product of two colored Brauer diagrams is defined to be a certain multiple of another colored Brauer diagram, determined as follows.

Given two colored Brauer diagrams $a, b$, first "stack" $b$ over $a$ (as for tangle diagrams and ordinary Brauer diagrams). In the resulting "tangle" there are three types of curves:

(1) Vertical strands. These are concatenations of one vertical strand from $b$, an even number of horizontal strands from the bottom of $b$ and the top of $a$, and finally one vertical strand from $a$. Traversing such a composite strand in its standard orientation (from lower numbered vertex to higher numbered vertex) sum the integer values of the oriented strands encountered. (We repeat for emphasis: if a strand colored by $r$ is traversed in the negative direction, then it contributes $-r$ to the sum.) Color the strand by the resulting sum.

(2) Horizontal strands. These include horizontal strands remaining from the original diagrams, namely horizontal strands from the top of $b$, and from the bottom of $a$. These strands retain their original coloring from the original diagrams. The horizontal strands also include concatenations of a vertical strand from $b$ or $a$ followed by an odd number of horizontal strands from the bottom of $b$ and the top of $a$, and finally a second vertical strand from the same diagram as the first vertical strand. The color of the composite strand is determined as for composite vertical strands.

(3) Closed strands. These are concatenations of an even number of horizontal strands from the bottom of $b$ and the top of $a$. There is no preferred orientation on such a strand, so pick an orientation arbitrarily, and obtain a color by summing the integer values of the oriented strands encountered in traversing the curve, as for vertical strands. For each $i \in\{0,1, \ldots\}$, let $m_{i}$ be the number of closed loops with color $\pm i$.

Let $c$ be the colored Brauer diagram obtained by removing all the closed curves. Then

$$
a b=\left(\boldsymbol{\delta}^{m_{0}} \boldsymbol{q}_{1}^{m_{1}} \boldsymbol{q}_{2}^{m_{2}} \cdots\right) c .
$$

Definition 4.4. The affine Brauer algebra $\widehat{D}_{n}$ over $\mathbb{Z}\left[\boldsymbol{\delta}^{ \pm 1}\right]^{\wedge}$ is the free $\mathbb{Z}\left[\boldsymbol{\delta}^{ \pm 1}\right]^{\wedge}$-module with basis the set of colored $(n, n)$-Brauer diagrams, with the bilinear product determined by the multiplication of colored Brauer diagrams. 
One can easily check that the multiplication is associative. Note that the subalgebra generated by colored Brauer diagrams with only vertical strands is isomorphic to the wreath product of $\mathbb{Z}$ with $\mathfrak{S}_{n}$.

\subsection{Conditional expectation and trace for the affine Brauer} algebras. Just as for affine Kauffman tangle algebras, one has a homomorphism $\iota$ of $\widehat{D}_{n-1}$ into $\widehat{D}_{n}$ by attaching an additional strand on the right of an affine Brauer diagram (colored by 0 ).

Moreover, one has a conditional expectation $\varepsilon_{n}: \widehat{D}_{n} \rightarrow \widehat{D}_{n-1}$ defined as follows: First define a map $\mathrm{cl}_{n}$ from colored $n$-connectors to colored $(n-1)$ connectors by joining the rightmost pair of vertices $\bar{n}, \boldsymbol{n}$ of a colored $n$ connector $d$ by a new strand, with color 0 :

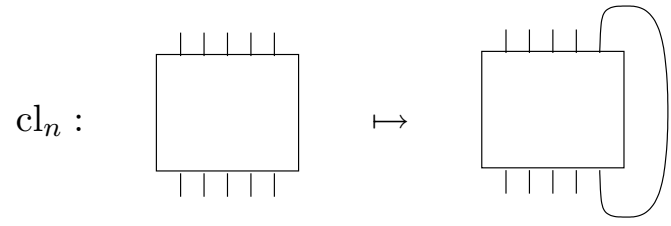

The new strand is part of a concatenated (vertical, horizontal or closed) strand. If the concatenated strand is vertical or horizontal, orient it positively (from lower numbered vertex to higher) and obtain its color by adding the colors of its (oriented) components. If the concatenated strand is closed (which happens precisely if $d$ contains a strand connecting $\boldsymbol{n}$ and $\overline{\boldsymbol{n}}$ with some color $r$ ) then remove the closed loop and multiply the resulting colored $(n-1)$-connector by $\boldsymbol{q}_{|r|}$ if $r \neq 0$ or $\boldsymbol{\delta}$ if $r=0$. For example:

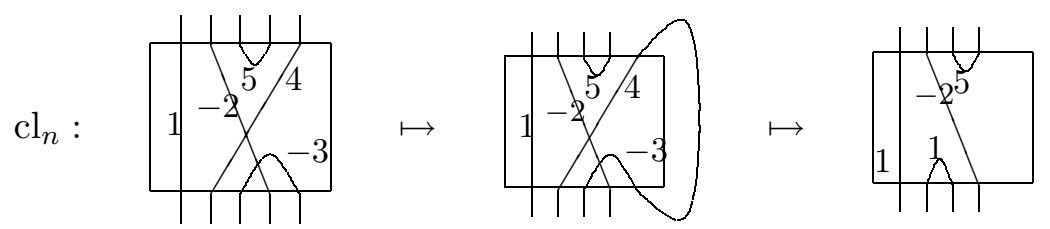

Define $\varepsilon_{n}: \widehat{D}_{n} \rightarrow \widehat{D}_{n-1}$ by

$$
\varepsilon_{n}(d)=\delta^{-1} \operatorname{cl}_{n}(d)
$$

One can check that $\varepsilon_{n}$ is a conditional expectation. Since $\varepsilon_{n} \circ \iota(x)=x$, the map $\iota$ is injective; therefore, we consider $\widehat{D}_{n-1}$ as a subalgebra of $\widehat{D}_{n}$. 
Define $\varepsilon=\varepsilon_{1} \circ \cdots \circ \varepsilon_{n}: \widehat{D}_{n} \rightarrow \widehat{D}_{0}=\mathbb{Z}\left[\boldsymbol{\delta}^{ \pm 1}\right]^{\wedge}$. Alternatively, define the closure cl of a colored $n$-connector by closing all the strands:

$\mathrm{cl}$ :
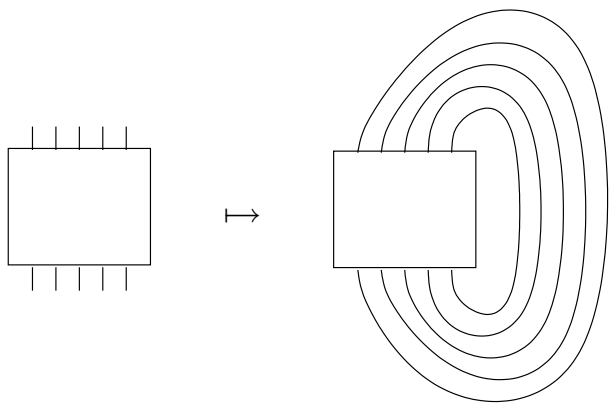

Compute the color of each (closed) strand in the resulting diagram as before, and replace each closed strand by the appropriate factor $\boldsymbol{q}_{r}$ or $\boldsymbol{\delta}$. Then $\varepsilon(d)=\boldsymbol{\delta}^{-n} \operatorname{cl}(d)$.

Using this picture for $\varepsilon$, one can check that $\varepsilon$ is a trace.

We can define a symmetric $\mathbb{Z}\left[\boldsymbol{\delta}^{ \pm 1}\right]^{\wedge}$-bilinear form on $\widehat{D}_{n}$ by $(x, y) \mapsto$ $\varepsilon(x y)$.

Define the reflection $\bar{d}$ of a colored $n$-connector $d$ by reflecting the diagram vertically and changing the color of each strand to its opposite:

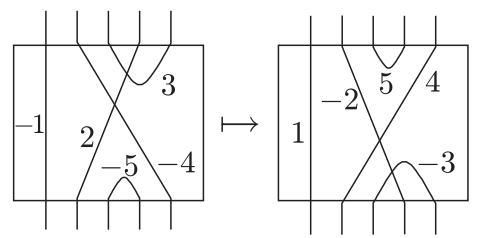

Note that for colored $n$-connectors $d, d^{\prime}$, the closure $\operatorname{cl}\left(d d^{\prime}\right)$ has at most $n$ (closed) strands, and that it has $n$ precisely when the underlying diagrams of $d$ and $d^{\prime}$ are reflections of one another. Moreover, each of these closed loops has color 0 precisely when $d^{\prime}=\bar{d}$. Consequently, we have:

LEMMA 4.5.

(1) For colored $n$-connectors $d, d^{\prime}$,

$$
\varepsilon\left(d d^{\prime}\right)=\boldsymbol{\delta}^{-n} m,
$$

where $m$ is a monomial in $\boldsymbol{\delta}, \boldsymbol{q}_{1}, \boldsymbol{q}_{2}, \ldots$ of total degree $\leq n$. The degree of $\varepsilon\left(d d^{\prime}\right)$ in $\boldsymbol{\delta}$ is strictly negative unless $d^{\prime}=\bar{d}$, while $\varepsilon(d \bar{d})=1$.

(2) Let $S$ be a finite set of colored n-connectors that is closed under the reflection $d \mapsto \bar{d}$. Consider the matrix $A_{S}=\left(\varepsilon\left(d d^{\prime}\right)\right)_{d, d^{\prime} \in S}$. The determinant of $A_{S}$ is non-zero.

Proof. The first statement follows from the preceding discussion. For the second statement, each row and column of $A_{S}$ has exactly one entry 
equal to 1 . All other entries have strictly negative degree in $\boldsymbol{\delta}$. Therefore, $\operatorname{det}\left(A_{S}\right)= \pm 1+\boldsymbol{\delta}^{-1} p$, where $p$ is a polynomial in $\boldsymbol{\delta}^{-1}$ with coefficients in $\mathbb{Z}\left[\boldsymbol{q}_{1}, \boldsymbol{q}_{2}, \ldots\right]$.

4.4. A homomorphism from $\widehat{\mathrm{KT}}_{n, \widehat{\Lambda}}$ to $\widehat{D}_{n}$. We wish to define a map from affine tangle diagrams to colored Brauer diagrams which basically forgets the sense of crossings of ordinary strands, but remembers the sense of crossings of ordinary strands with the flagpole.

Define a map $c$ (the connector map) from affine $(n, n)$-tangle diagrams to $\widehat{D}_{n}$ as follows. Number and order the $2 n$ vertices of affine $(n, n)$-tangle diagrams by the same convention as for colored Brauer diagrams. For each strand of an affine $(n, n)$-tangle diagram $a$ that connects two vertices, draw a curve connecting the corresponding vertices in $c(a)$. Determine the color $r$ of the curve as follows: with the strand oriented from the lower numbered vertex to the higher numbered vertex, $r$ is the number of clockwise rotations of the strand around the flagpole, viewed from above $\left(^{1}\right)$.

Let $d$ be the resulting colored $n$-connector. Give each closed strand of $a$ an arbitrary orientation, and determine the color of the strand as above; for each $r \geq 1$, let $m_{r}$ be the number of closed loops in $a$ with color $\pm r$. Finally, define

$$
c(a)=\left(\boldsymbol{\delta}^{m_{0}} \boldsymbol{q}_{1}^{m_{1}} \boldsymbol{q}_{2}^{m_{2}} \cdots\right) d .
$$

The map $c$ respects regular isotopy (in fact, ambient isotopy), so induces a map $c: \widehat{\mathcal{U}}(n, n) \rightarrow \widehat{D}_{n}$. One can check that $c$ is a monoid map, so we extend it linearly to a $\mathbb{Z}\left[\boldsymbol{\delta}^{ \pm 1}\right]^{\wedge}$-algebra map

$$
c: \mathbb{Z}\left[\boldsymbol{\delta}^{ \pm 1}\right]^{\wedge} \widehat{\mathcal{U}}(n, n) \rightarrow \widehat{D}_{n}
$$

This algebra map respects the relations of $\widehat{\mathrm{KT}}_{n, \mathbb{Z}\left[\boldsymbol{\delta}^{ \pm 1}\right]^{\wedge}}$ (see Remark 2.12 at the the end of Section 2.4), so induces a map

$$
c: \widehat{\mathrm{KT}}_{n, \mathbb{Z}\left[\boldsymbol{\delta}^{ \pm 1}\right]^{\wedge}} \rightarrow \widehat{D}_{n}
$$

Finally, we have the composition

$$
c: \widehat{\mathrm{KT}}_{n, \widehat{\Lambda}} \rightarrow \widehat{\mathrm{KT}}_{n, \mathbb{Z}\left[\boldsymbol{\delta}^{ \pm 1}\right]^{\wedge}} \rightarrow \widehat{D}_{n} .
$$

This map $c$ is given simply by the formula $c\left(\sum \alpha_{i} T_{i}\right)=\sum e\left(\alpha_{i}\right) c\left(T_{i}\right)$, where $\alpha_{i} \in \widehat{\Lambda}$, the $T_{i}$ are affine tangles representing elements of $\widehat{\mathrm{KT}}_{n, \widehat{\Lambda}}$, and $e$ is the homomorphism of $\widehat{\Lambda}$ to $\mathbb{Z}\left[\boldsymbol{\delta}^{ \pm 1}\right]^{\wedge}$ determined by $\boldsymbol{\lambda} \mapsto 1, z \mapsto 0, \boldsymbol{\delta} \mapsto \boldsymbol{\delta}$, and $\boldsymbol{q}_{i} \mapsto \boldsymbol{q}_{i}$.

$\left({ }^{1}\right)$ The color $r$ can be determined combinatorially: traversing the strand from lower numbered vertex to higher numbered vertex, list the over-crossings $(+)$ and undercrossings $(-)$ of the strand with the flagpole. Cancel any two successive +'s or -'s in the list, so the list now consists of alternating +'s and -'s. Then $r$ is $\pm(1 / 2)$ the length of the list, + if the list begins with $\mathrm{a}+$, and - if the list begins with $\mathrm{a}-$. 
Proposition 4.6. The following diagrams commute:

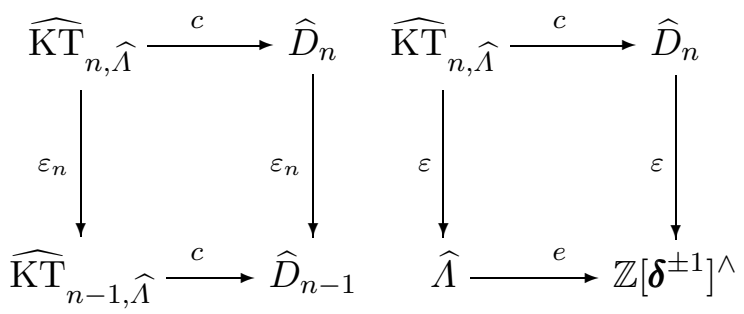

Proof. Left to the reader.

The following proposition is adapted from [9] and [10].

Proposition 4.7. For each colored n-connector $d$, let $T_{d}$ be an affine $(n, n)$-tangle diagram with $c\left(T_{d}\right)=d$. Then the set $\left\{T_{d}: d\right.$ a colored $n$ connector $\}$ is linearly independent over $\widehat{\Lambda}$.

Proof. Let $S$ be a finite set of colored $n$-connectors which is closed under reflection $d \mapsto \bar{d}$. Consider the matrices $B_{S}=\left(\varepsilon\left(T_{d} T_{d^{\prime}}\right)\right)_{d, d^{\prime} \in S}$ and $A_{S}=$ $\left(\varepsilon\left(d d^{\prime}\right)\right)_{d, d^{\prime} \in S}$. One has $e\left(\operatorname{det}\left(B_{S}\right)\right)=\operatorname{det}\left(e \circ \varepsilon\left(T_{d} T_{d^{\prime}}\right)\right)=\operatorname{det}\left(\varepsilon \circ c\left(T_{d} T_{d^{\prime}}\right)\right)=\operatorname{det}\left(\varepsilon\left(d d^{\prime}\right)\right)=\operatorname{det}\left(A_{S}\right)$. By Lemma $4.5, \operatorname{det}\left(A_{S}\right) \neq 0, \operatorname{so} \operatorname{det}\left(B_{S}\right) \neq 0$. Since $\widehat{\Lambda}$ is an integral domain, $B_{S}$ is invertible over the field of fractions of $\widehat{\Lambda}$. It follows that $\left\{T_{d}: d \in S\right\}$ is linearly independent over $\widehat{\Lambda}$.

Since $S$ is arbitrary, it follows that $\left\{T_{d}: d\right.$ a colored $n$-connector $\}$ is linearly independent over $\widehat{\Lambda}$.

COROLlary 4.8. For each ordinary n-connector $d$, let $T_{d}$ be an ordinary $(n, n)$-tangle diagram with $c\left(T_{d}\right)=d$. Then the set $\left\{T_{d}: d\right.$ an $n$-connector $\}$ is linearly independent over $\Lambda$.

Proof. Use the injection $i_{n}: \mathrm{KT}_{n, \Lambda} \rightarrow \widehat{\mathrm{KT}}_{n, \widehat{\Lambda}}$ of Remark 2.11 .

\section{ISOMORPHISM OF}

\section{ORDINARY BMW AND KAUFFMAN TANGLE ALGEBRAS}

This section is devoted to exhibiting an $R$-basis of $\mathrm{KT}_{n, R}$ and to proving that $\varphi: W_{n, R} \rightarrow \mathrm{KT}_{n, R}$ is an isomorphism. The results and arguments of this section are taken from [10].

All the tangle diagrams in this section will be ordinary $(n, n)$-tangle diagrams for some $n$.

\subsection{Totally descending tangles and freeness of $\mathrm{KT}_{n}$}

LEMma 5.1. $\mathrm{KT}_{n, R}$ is spanned by tangle diagrams without closed strands. 
Proof. The proof is by induction on the number of crossings.

If a tangle diagram $T$ has no crossings, then, by Definition 2.6(3), $T=$ $\delta^{k} T^{\prime}$, where $k$ is the number of closed loops of $T$ and $T^{\prime}$ is the tangle diagram obtained by removing all the closed loops of $T$.

Let $T$ be a tangle diagram with $l \geq 1$ crossings. Assume that any tangle diagram with fewer than $l$ crossings is in the span of tangle diagrams without closed strands.

By the Kauffman tangle relation, if $S$ is a tangle diagram which differs from $T$ only by reversing one or more crossings, then $T$ and $S$ are congruent modulo the span of tangle diagrams with fewer crossings. Hence $T$ and $S$ are congruent modulo the span of tangle diagrams without closed strands, by the induction hypothesis.

Suppose $T$ has a closed strand $s$. By changing crossings, one can suppose that $s$ has only over-crossings with other strands of $T$ and that $s$ is unknotted. Then $T$ is ambient isotopic to a tangle diagram in which the closed strand corresponding to $s$ has no crossings with other strands and no self-crossings. If $T^{\prime}$ is the tangle diagram with $s$ removed, then $T=\delta \lambda^{k} T^{\prime}$ for some $k$, by Definition $2.6(2,3)$.

DEFINITION 5.2. An orientation of an affine or ordinary $(n, n)$-tangle diagram is a linear ordering of the strands, a choice of an orientation of each strand, and a choice of an initial point on each closed loop. by

Order the boundary points $\{1, \ldots, \boldsymbol{n}, \overline{\mathbf{1}}, \ldots, \overline{\boldsymbol{n}}\}$ of $(n, n)$-tangle diagrams

$$
\mathbf{1}<\mathbf{2}<\cdots<\boldsymbol{n}<\overline{\boldsymbol{n}}<\cdots<\overline{\mathbf{2}}<\overline{\mathbf{1}},
$$

as in the discussion of colored $n$-connectors.

Definition 5.3. A standard orientation of an ordinary or affine $(n, n)$ tangle diagram is one in which

(1) each non-closed strand is oriented from its lower numbered endpoint to its higher numbered endpoint,

(2) the non-closed strands are ordered according to the order of their initial endpoints,

(3) the closed loops follow the non-closed strands in the ordering of the strands.

If a tangle diagram has no closed loops, then it has a unique standard orientation.

An orientation determines a way of traversing the tangle diagram; namely, the strands are traversed successively, in the given order and orientation (the closed loops being traversed starting at the assigned initial point). 
Definition 5.4. An oriented ordinary $(n, n)$-tangle diagram is totally descending with respect to its orientation if, as the tangle diagram is traversed, each crossing is encountered first as an over-crossing.

Proposition 5.5. $\mathrm{KT}_{n, R}$ is spanned by ordinary $(n, n)$-tangle diagrams without closed loops that are totally descending with respect to the standard orientation.

Proof. We already know that $\mathrm{KT}_{n, R}$ is spanned by $(n, n)$-tangle diagrams without closed loops.

If a tangle diagram has no crossings, it is already totally descending. Let $T$ be a tangle diagram with $l \geq 1$ crossings. Assume that any tangle diagram with fewer than $l$ crossings is in the span of totally descending tangle diagrams. The totally descending tangle diagram $S$ which differs from $T$ only by reversing some number of crossings is congruent to $T$ modulo the span of tangle diagrams with fewer crossings, hence modulo the span of totally descending tangle diagrams.

COROLlary 5.6. $\mathrm{KT}_{n, R}$ is spanned by the set of $(n, n)$-tangle diagrams $T$ without closed loops that are totally descending with respect to the standard orientation, and such that no strand of $T$ has self-crossings.

Proof. If $T$ is a totally descending $(n, n)$-tangle diagram, then $T$ is layered; that is, $T$ can be drawn with different strands in different levels above the plane of $R=\mathbb{R} \times I$. The individual strands are unknotted, so they can be changed by level-preserving ambient isotopy to arcs without self-crossings. Thus $T=\lambda^{k} T^{\prime}$, where $T^{\prime}$ is a totally descending tangle whose strands have no self-crossings.

Proposition 5.7. Suppose $S$ and $T$ are two $(n, n)$-tangle diagrams without closed loops such that

(1) $S$ and $T$ have the same connector,

(2) $S$ and $T$ are both totally descending (with respect to the same orientation),

(3) the strands of $S$ and $T$ have no self-crossings.

Then $S$ and $T$ are regularly isotopic, so they represent the same element of $\mathrm{KT}_{n, R}$.

Proof. Since $S$ and $T$ have the same connector and are both totally descending with respect to the same ordering of the strands, they can be layered, with the strands connecting corresponding endpoints in the two diagrams lying at the same level above the plane of $R$. Each strand of $S$ can then be deformed by a level-preserving isotopy to coincide with the corresponding strand of $T$; this deformation corresponds to regular isotopy of the diagrams. 
For each $n$-connector $d$, let $T_{d}$ be an $(n, n)$-tangle diagram without closed loops such that

(1) $c\left(T_{d}\right)=d$,

(2) $T_{d}$ is totally descending with respect to the standard orientation,

(3) the strands of $T_{d}$ have no self-crossings.

By Proposition 5.7, $T_{d}$ is unique up to regular isotopy. The tangle diagrams $T_{d}$ (or rather the regular isotopy classes which they represent) can be regarded as elements of $\mathrm{KT}_{n, R}$ for any $R$. For any $R$,

$$
B_{0}=\left\{T_{d}: d \text { is an } n \text {-connector }\right\}
$$

spans $\mathrm{KT}_{n, R}$, by Corollary 5.6. By Corollary $4.8, B_{0}$ is linearly independent in $\mathrm{KT}_{n, \Lambda}$. Thus we have:

THEOREM 5.8. $\mathrm{KT}_{n, \Lambda}$ is free over $\Lambda$ with basis $B_{0}$.

COROLlary 5.9. For each $R, \mathrm{KT}_{n, R}$ is free over $R$ with basis $B_{0}$. Moreover, $\mathrm{KT}_{n, R} \cong \mathrm{KT}_{n, \Lambda} \otimes_{\Lambda} R$.

Proof. $\mathrm{KT}_{n, \Lambda} \otimes_{\Lambda} R$ is free over $R$ with basis $B_{0} \otimes 1$. On the other hand, $B_{0}$ spans $\mathrm{KT}_{n, R}$ by Corollary 5.6. There is an $R$-algebra homomorphism from $\mathrm{KT}_{n, R}$ to $\mathrm{KT}_{n, \Lambda} \otimes_{\Lambda} R$ which sends a tangle $T$ to $T \otimes 1$. Since this map takes a spanning set to a basis, it is an isomorphism.

COROLlary 5.10. Let $R$ be a ring with distinguished elements $\lambda, z$ and $\delta$, and let $S \supseteq R$ be a ring containing $R$. Then $\mathrm{KT}_{n, R}$ imbeds in $\mathrm{KT}_{n, S}$.

Corollary 5.11. Let $R$ be a ring with distinguished elements $\lambda$, $z$, and $\delta$, and $S \supseteq R$ a ring with additional elements $q_{1}, q_{2}, \ldots$ The $R$-algebra homomorphism $i_{n}: \mathrm{KT}_{n, R} \rightarrow \widehat{\mathrm{KT}}_{n, S}$ of Remark 2.11 is injective.

Proof. This follows from the previous corollary and point (1) of Remark 2.11 .

\subsection{Positive permutation braids}

DeFinition 5.12. An $n$-braid diagram is an $(n, n)$-tangle diagram all of whose strands are monotone. That is, each strand decreases monotonically from a top vertex to a bottom vertex.

Definition 5.13. The (geometric) braid group $B_{n}$ is the group of $n$ braid diagrams modulo ambient isotopy.

The group $B_{n}$ has the well known presentation (due to Artin) with generators $\sigma_{1}, \ldots, \sigma_{n-1}$ and relations

(1) $\sigma_{i} \sigma_{i+1} \sigma_{i}=\sigma_{i+1} \sigma_{i} \sigma_{i+1}$,

(2) $\sigma_{i} \sigma_{j}=\sigma_{j} \sigma_{i}$ if $|i-j| \geq 2$. 
The generator $\sigma_{i}$ is the $n$-braid diagram with a single positive crossing between the $i$ th and $(i+1)$ st strand,

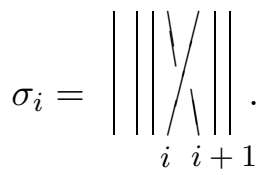

Since the generators $g_{i}$ of the ordinary BMW algebra satisfy the braid relations, $\psi: \sigma_{i} \mapsto g_{i}$ determines a group homomorphism from $B_{n}$ into the group of invertible elements of $W_{n}$. Denote by perm the homomorphism from $B_{n}$ to the symmetric group $\mathfrak{S}_{n}: \operatorname{perm}(\beta)(i)=j$ if the braid diagram $\beta$ connects the top vertex $\boldsymbol{i}$ with the bottom vertex $\overline{\boldsymbol{j}}$. In particular $\operatorname{perm}\left(\sigma_{i}\right)$ is the adjacent transposition $s_{i}=(i, i+1)$. Note that perm $=c \circ \varphi \circ \psi$, where we identify permutations with their diagrams in the Brauer algebra,

$$
\text { perm : } \sigma_{i} \mapsto g_{i} \mapsto G_{i} \mapsto c\left(G_{i}\right)=s_{i} .
$$

Proposition 5.14. The following are equivalent for an element $\beta$ of the braid group:

(1) Two strands of $\beta$ cross at most once, and all crossings are positive (that is, $\beta$ is in the monoid generated by the $\sigma_{i}$ ).

(2) $\beta$ is the product of $r$ generators $\sigma_{i}$, where $r$ is the length of $\operatorname{perm}(\beta)$. Moreover, if $\beta$ satisfies these conditions, and $s_{i_{r}} \cdots s_{i_{1}}$ is any reduced expression for $\operatorname{perm}(\beta)$, then $\beta=\sigma_{i_{r}} \cdots \sigma_{i_{1}}$.

Proof. Suppose that $\beta=\sigma_{i_{r}} \cdots \sigma_{i_{1}}$, but that two strands of $\beta$ cross twice. Then $\operatorname{perm}(\beta)=s_{i_{r}} \cdots s_{i_{1}}$ has a subword $s_{a} \pi_{0} s_{b}$ with the property that $\pi_{0}(b)=a$ and $\pi_{0}(b+1)=a+1$. But then $\pi_{0} s_{b}=s_{a} \pi_{0}$, so $s_{a} \pi_{0} s_{b}=\pi_{0}$, and the length of $\operatorname{perm}(\beta)$ is less than $r$. This proves $(2) \Rightarrow(1)$.

Now suppose that $\beta$ satisfies (1). Let $\operatorname{perm}(\beta)=s_{i_{r}} \cdots s_{i_{1}}$ be a reduced expression for $\operatorname{perm}(\beta)$ and set $\beta^{\prime}=\sigma_{i_{r}} \cdots \sigma_{i_{1}}$. Then $\beta$ and $\beta^{\prime}$ are two braid diagrams both satisfying condition (1) with $\operatorname{perm}(\beta)=\operatorname{perm}\left(\beta^{\prime}\right)$. But a braid diagram satisfying condition (1) is totally descending (with respect to the orientation in which the strands are oriented from top to bottom and ordered in the reversed order of their top vertices). Therefore, by Proposition 5.7, such a braid diagram $\beta$ is determined up to ambient isotopy by its connector, that is, by $\operatorname{perm}(\beta)$.

Definition 5.15. A braid diagram satisfying the conditions of the previous proposition is called a positive permutation braid.

For each $\pi \in \mathfrak{S}_{n}$, there is a unique positive permutation braid $\beta_{\pi} \in B_{n}$ with $\operatorname{perm}\left(\beta_{\pi}\right)=\pi$. We write $g_{\pi}$ for the image of $\beta_{\pi}$ in $W_{n}$ and $G_{\pi}$ for the image of $\beta_{\pi}$ in $\mathrm{KT}_{n}$. We also call these elements (which are determined by $\pi$ ) positive permutation braids. Note that $g_{\pi^{-1}}=\alpha\left(g_{\pi}\right)$. 
For $\pi \in \mathfrak{S}_{n}$ and $1 \leq i<n$, we have $\ell\left(\pi s_{i}\right)=\ell(\pi)+1 \Leftrightarrow \pi(i)<\pi(i+1)$. In this case, $g_{\pi s_{i}}=g_{\pi} g_{i}$. Otherwise, $\ell\left(\pi s_{i}\right)=\ell(\pi)-1, \pi(i)>\pi(i+1)$, and $g_{\pi s_{i}}=g_{\pi} g_{i}^{-1}$. Likewise, $\ell\left(s_{i} \pi\right)=\ell(\pi)+1 \Leftrightarrow \pi^{-1}(i)<\pi^{-1}(i+1)$. In this case, $g_{s_{i} \pi}=g_{i} g_{\pi}$. Otherwise, $\ell\left(\pi s_{i}\right)=\ell(\pi)-1, \pi^{-1}(i)>\pi^{-1}(i+1)$, and $g_{s_{i} \pi}=g_{i}^{-1} g_{\pi}$.

Definition 5.16. An $(a, b)$-shuffle is an element $\pi \in \mathfrak{S}_{a+b}$ such that $\pi(i)<\pi(j)$ if $1 \leq i<j \leq a$ or $a+1 \leq i<j \leq a+b$.

LEMmA 5.17. If $\pi \in \mathfrak{S}_{a+b}$ then $\pi=\pi_{1} \pi_{2}$ where $\pi_{1}$ is an $(a, b)$-shuffle, $\pi_{2} \in \mathfrak{S}_{a} \times \mathfrak{S}_{b} \subseteq \mathfrak{S}_{a+b}$, and $\ell(\pi)=\ell\left(\pi_{1}\right)+\ell\left(\pi_{2}\right)$. It follows that $g_{\pi}=g_{\pi_{1}} g_{\pi_{2}}$.

Proof. The proof is by induction on the length of $\pi$. The result is evident if $\pi$ is the identity permutation. If $\pi$ is not already an $(a, b)$-shuffle, then there exists an $i$ with $i \neq a$ such that $\pi(i)>\pi(i+1)$. Consequently, $\pi=\pi^{\prime} s_{i}$ with $\ell(\pi)=\ell\left(\pi^{\prime}\right)+1$. The result follows by applying the induction hypothesis to $\pi^{\prime}$.

Lemma 5.18. Let $1 \leq i \leq n-1$ and suppose $\pi \in \mathfrak{S}_{n}$ satisfies $\pi^{-1}(i+1)=$ $\pi^{-1}(i)+1$. Then $g_{i} g_{\pi}=g_{\pi} g_{\pi^{-1}(i)}$ and $e_{i} g_{\pi}=g_{\pi} e_{\pi^{-1}(i)}$.

Proof. Put $j=\pi^{-1}(i)$. We have $s_{i} \pi=\pi s_{j}$, and $\ell\left(s_{i} \pi\right)=\ell(\pi)+1$. Hence

$$
g_{\pi} g_{j}=g_{\pi s_{j}}=g_{s_{i} \pi}=g_{i} g_{\pi} \text {. }
$$

The second equality is proved by induction on the length of $\pi$. If $\ell(\pi)=0$, the assertion is trivial. Suppose that $\ell(\pi)>1$. Choose $k$ such that $\pi=s_{k} \pi_{1}$, and $\ell(\pi)=\ell\left(\pi_{1}\right)+1$. To prove the induction step, we consider three cases:

(1) $k=i+1$. In this case $\pi^{-1}(i+2)<\pi^{-1}(i+1)=\pi^{-1}(i)+1$. It follows that $\pi^{-1}(i+2)<\pi^{-1}(i)$, and $\pi=s_{i+1} s_{i} \pi^{\prime}$, with $\ell(\pi)=\ell\left(\pi^{\prime}\right)+2$. Therefore $g_{\pi}=g_{i+1} g_{i} g_{\pi^{\prime}}$ and $e_{i} g_{\pi}=e_{i} g_{i+1} g_{i} g_{\pi}^{\prime}=g_{i+1} g_{i} e_{i+1} g_{\pi^{\prime}}$. Since $\pi^{\prime-1}(i+2)=j+1=\pi^{\prime-1}(i+1)+1$, we have $e_{i+1} g_{\pi^{\prime}}=g_{\pi^{\prime}} e_{j}$, by the induction hypothesis.

(2) $k=i-1$. This case is similar.

(3) $|k-i| \geq 2$. Then $e_{i} g_{\pi}=e_{i} g_{k} g_{\pi_{1}}=g_{k} e_{i} g_{\pi_{1}}$. Now $\pi_{1}{ }^{-1}(i+1)=j+1=$ $\pi_{1}^{-1}(i)+1$, so by the induction hypothesis, $e_{i} g_{\pi_{1}}=g_{\pi_{1}} e_{j}$.

5.3. Surjectivity of $\varphi: W_{n} \rightarrow \mathrm{KT}_{n}$. We will prove that $\mathrm{KT}_{n, R}$ is generated as a unital algebra by $\left\{E_{i}, G_{i}^{ \pm 1}: 1 \leq i \leq n-1\right\}$, which is equivalent to the surjectivity of $\varphi: W_{n, R} \rightarrow \mathrm{KT}_{n, R}$.

The tensor product $T_{1} \otimes T_{2}$ of a $(k, k)$-tangle diagram and an $(l, l)$-tangle diagram is the $(k+l, k+l)$-tangle diagram obtained by placing $T_{1}$ and $T_{2}$ side by side.

The tensor product of tangle diagrams clearly extends to a bilinear product $\mathrm{KT}_{k, R} \times \mathrm{KT}_{l, R} \rightarrow \mathrm{KT}_{k+l, R}$. If $T_{1}$ and $T_{2}$ are both in the unital subalgebra generated by the $E_{i}$ 's and $G_{i}$ 's, then so is $T_{1} \otimes T_{2}$. 
Lemma 5.19. Any element of $\mathrm{KT}_{n, R}$ which is represented by an $n$-braid diagram is in the group generated by $\left\{G_{i}^{ \pm 1}: 1 \leq i \leq n-1\right\}$.

Proof. Induction on the number of crossings.

TheOREM 5.20. $\varphi: W_{n, R} \rightarrow \mathrm{KT}_{n, R}$ is surjective.

Proof. For $n=0$ and $n=1, \mathrm{KT}_{n, R} \cong R$ by Corollary 5.9, and the statement is trivially valid.

Fix $n \geq 2$. We have to show that $\mathrm{KT}_{n, R}$ is generated as a unital algebra by $\left\{E_{i}, G_{i}^{ \pm 1}: 1 \leq i \leq n-1\right\}$. By Theorem 5.8 , it suffices to show that each totally descending $(n, n)$-tangle diagram $T$ whose strands have no selfcrossings is in the unital subalgebra generated by the $E_{i}$ 's and $G_{i}$ 's.

If the connector $c(T)$ is a permutation diagram (i.e. top vertices are connected only to bottom vertices), then $T$ is regularly isotopic to an $n$-braid diagram, and thus $T$ is in the monoid generated by $\left\{G_{i}^{ \pm 1}: 1 \leq i \leq n-1\right\}$.

Otherwise, for some $k \geq 1$, the connector of $T$ has $k$ horizontal strands connecting pairs of vertices at the top and $k$ horizontal strands connecting vertices at the bottom. In this case there exist $n$-braid diagrams $B_{1}$ and $B_{2}$ and an $(n-2 k)$-braid diagram $B$ such that

$$
T=B_{1}\left[\left(E_{1} E_{3} \cdots E_{2 k-1}\right) \otimes B\right] B_{2} .
$$

Since each of $B_{1}, B_{2}$, and $B$ are in the group generated by the $\left\{G_{i}^{ \pm 1}\right\}$, it follows that $T$ is in the monoid generated by $\left\{E_{i}, G_{i}^{ \pm 1}\right\}$.

5.4. The elements $f_{k}$ and a filtration of $W_{n}$. Fix a ring $R$ and write $W_{n}$ for $W_{n, R}$ and $\mathrm{KT}_{n}$ for $\mathrm{KT}_{n, R}$.

Consider the element $F_{k} \in \mathrm{KT}_{2 k}$ represented by the tangle diagram with no crossings, in which the points $\boldsymbol{i}$ and $\mathbf{2} \boldsymbol{k}+\mathbf{1}-\boldsymbol{i}$ at the top of the diagram

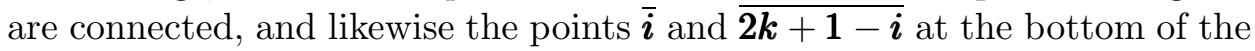
diagram are connected. For example

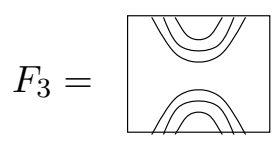

The element $F_{k}$ is evidently fixed by the symmetries $\alpha, \beta$, and $\varrho_{2 k}$ of $\mathrm{KT}_{2 k}$.

The following proposition is from [10] and is easily verified by picture proofs.

Proposition 5.21.

(1) For all $i<k, G_{i}^{ \pm 1} F_{k}=G_{2 k-i}^{ \pm 1} F_{k}$ and $F_{k} G_{i}^{ \pm 1}=F_{k} G_{2 k-i}^{ \pm 1}$.

(2) For all $i<k, E_{i} F_{k}=E_{2 k-i} F_{k}$ and $F_{k} E_{i}=F_{k} E_{2 k-i}$.

(3) $F_{k}=\left(G_{1} G_{2} \cdots G_{2 k-1}\right) F_{k-1} E_{2 k-1}\left(G_{2 k-1} \cdots G_{2} G_{1}\right)$. 
Following [10], we recursively define elements $f_{k}$ of $W_{2 k}$ such that $\varphi\left(f_{k}\right)$ $=F_{k}$, as follows:

Definition 5.22. Define $f_{1}=e_{1}$ and

$$
f_{k}=\left(g_{1} g_{2} \cdots g_{2 k-2}\right) f_{k-1} e_{2 k-1}\left(g_{2 k-2} \cdots g_{2} g_{1}\right)
$$

for $k \geq 2$.

We want to find an expression for $f_{k}$ that does not involve the $g_{i}$ 's (since the corresponding tangle diagrams have no crossings), and that makes manifest the symmetries $\alpha\left(f_{k}\right)=\beta\left(f_{k}\right)=\varrho_{2 k}\left(f_{k}\right)=f_{k}$ (which the definition does not).

LEMMA 5.23.

$$
\begin{aligned}
& \left(g_{1} g_{2} \cdots g_{2 k}\right)\left(e_{1} e_{3} \cdots e_{2 k-1}\right)=\left(e_{2} e_{4} \cdots e_{2 k}\right)\left(e_{1} e_{3} \cdots e_{2 k-1}\right), \\
& \left(e_{1} e_{3} \cdots e_{2 k-1}\right)\left(g_{2 k} \cdots g_{2} g_{1}\right)=\left(e_{1} e_{3} \cdots e_{2 k-1}\right)\left(e_{2} e_{4} \cdots e_{2 k}\right) .
\end{aligned}
$$

Proof. The second equation follows from the first by applying the antiautomorphism $\alpha$. To prove the first equation, rewrite the left hand side as

$$
\left(g_{1} g_{2} e_{1}\right)\left(g_{3} g_{4} e_{3}\right) \cdots\left(g_{2 k-1} g_{2 k} e_{2 k-1}\right),
$$

which equals

$$
\left(e_{2} e_{1}\right)\left(e_{4} e_{3}\right) \cdots\left(e_{2 k} e_{2 k-1}\right),
$$

by use of $(5 \mathrm{~b})$ of Definition 3.6. Finally, the even terms can be shuffled to the left.

Proposition 5.24. For $k \geq 1$,

$$
f_{k}=\left(e_{k}\right)\left(e_{k-1} e_{k+1}\right) \cdots\left(e_{1} e_{3} \cdots e_{2 k-1}\right) \cdots\left(e_{k-1} e_{k+1}\right)\left(e_{k}\right) .
$$

For example,

$$
f_{4}=e_{4}\left(e_{3} e_{5}\right)\left(e_{2} e_{4} e_{6}\right)\left(e_{1} e_{3} e_{5} e_{7}\right)\left(e_{2} e_{4} e_{6}\right)\left(e_{3} e_{5}\right) e_{4} .
$$

Proof. The proof goes by induction on $k$, the assertion being evident for $k=1$. Consider $k>2$. We have

$$
\begin{aligned}
& f_{k}=\left(g_{1} g_{2} \cdots g_{2 k-2}\right) f_{k-1} e_{2 k-1}\left(g_{2 k-2} \cdots g_{2} g_{1}\right) \\
&=\left.\left(g_{1} g_{2} \cdots g_{2 k-2}\right)\left[e_{k-1}\left(e_{k-2} e_{k}\right) \cdots\left(e_{1} e_{3} \cdots e_{2 k-3}\right) \cdots\left(e_{k-2} e_{k}\right) e_{k-1}\right)\right] \\
& \cdot e_{2 k-1}\left(g_{2 k-2} \cdots g_{2} g_{1}\right)
\end{aligned}
$$

by the induction hypothesis. Move $e_{2 k-1}$ to the left to get

$$
\begin{array}{r}
\left.f_{k}=\left(g_{1} g_{2} \cdots g_{2 k-2}\right)\left[e_{k-1}\left(e_{k-2} e_{k}\right) \cdots\left(e_{1} e_{3} \cdots e_{2 k-3} e_{2 k-1}\right) \cdots\left(e_{k-2} e_{k}\right) e_{k-1}\right)\right] \\
\cdot\left(g_{2 k-2} \cdots g_{2} g_{1}\right) .
\end{array}
$$


Now move all of the $e_{i}$ 's, except those in the product $\left(e_{1} e_{3} \cdots e_{2 k-1}\right)$, to the left or right past a string of $g_{i}$ 's, making use of Lemma 3.9; this yields

$$
\begin{aligned}
f_{k}=e_{k}\left(e_{k-1} e_{k+1}\right) \cdots & \left(e_{3} e_{5} \cdots e_{2 k-3}\right)\left(g_{1} g_{2} \cdots g_{2 k-2}\right)\left(e_{1} e_{3} \cdots e_{2 k-3} e_{2 k-1}\right) \\
& \cdot\left(g_{2 k-2} \cdots g_{2} g_{1}\right)\left(e_{3} e_{5} \cdots e_{2 k-3}\right) \cdots\left(e_{k-1} e_{k+1}\right) e_{k} .
\end{aligned}
$$

Both strings of $g_{i}$ 's in the middle of the expression can be replaced by $e_{2} e_{4} \cdots e_{2 k-2}$, by the previous lemma, and this gives the desired expression.

REMARK 5.25. The elements in each group (indicated by parentheses) commute. It can be helpful to view the entire expression as a diamondshaped grid, for example

$$
f_{3}=e_{3}{ }^{e_{2}}{ }^{e_{3}}{ }^{e_{1}}{ }^{e_{2}} e_{3} e e_{4}
$$

This is read from left to right by columns, with the elements in each column commuting. This expression for $f_{k}$ makes evident the invariance of $f_{k}$ under the maps $\varrho_{2 k}, \alpha$, and $\beta$ of $W_{2 k}$.

Analogous elements in the Temperley-Lieb algebras were introduced in subfactor theory by M. Pimsner and S. Popa [16] to study iterations of the Jones basic construction; see also [7]. The diamond grid representation of these elements is due to A. Ocneanu (personal communication).

Note that one can also read the diamond grid by diagonals, so we have

$$
f_{k}=\left(e_{k} e_{k+1} \cdots e_{2 k-1}\right)\left(e_{k-1} e_{k} \cdots e_{2 k-2}\right) \cdots\left(e_{1} e_{2} \cdots e_{k}\right) .
$$

Moreover, $f_{k}$ satisfies

$$
f_{k}=\left(e_{k} e_{k+1} \cdots e_{2 k-2}\right) f_{k-1} e_{2 k-1}\left(e_{2 k-2} \cdots e_{k+1} e_{k}\right) .
$$

The following proposition from [10] establishes the analogue of Proposition 5.21 for the elements $f_{k}$. In points (3) and (4), $S$ denotes the shift homomorphism (see Remark 3.3).

Proposition 5.26.

(1) For $i<k, g_{i}^{ \pm 1} f_{k}=g_{2 k-i}^{ \pm 1} f_{k}$ and $f_{k} g_{i}^{ \pm 1}=f_{k} g_{2 k-i}^{ \pm 1}$.

(2) For $i<k, e_{i} f_{k}=e_{2 k-i} f_{k}$ and $f_{k} e_{i}=f_{k} e_{2 k-i}$.

(3) For $2 \leq i<k+1, g_{i}^{ \pm 1} S\left(f_{k}\right)=g_{2 k+2-i}^{ \pm 1} S\left(f_{k}\right)$ and $S\left(f_{k}\right) g_{i}^{ \pm 1}=$ $S\left(f_{k}\right) g_{2 k+2-i}^{ \pm 1}$.

(4) For $2 \leq i<k+1, e_{i} S\left(f_{k}\right)=e_{2 k+2-i} S\left(f_{k}\right)$ and $S\left(f_{k}\right) e_{i}=S\left(f_{k}\right) e_{2 k+2-i}$.

Proof. The second part of each assertion follows from the first part by applying the anti-automorphism $\alpha$. 
Note that $i$ and $2 k-i$ have the same parity, so $g_{i}$ and $g_{2 k-i}$ commute. Therefore, multiplying the equality

$$
g_{i} f_{k}=g_{2 k-i} f_{k}
$$

by $g_{i}^{-1} g_{2 k-i}^{-1}$ gives

$$
g_{i}^{-1} f_{k}=g_{2 k-i}^{-1} f_{k}
$$

Thus, for statement (1), it suffices to prove equation (5.1).

We prove statement (1) by induction on $k$ (following the proof in [10]). For $k=2$, the only instance to check is $i=1$. Using Lemma 3.9(3), we get

$$
g_{1} f_{2}=g_{1}\left(g_{1} g_{2} e_{1} e_{3}\right) g_{2} g_{1}=g_{1}\left(g_{3} g_{2} e_{3} e_{1}\right) g_{2} g_{1}=g_{3}\left(g_{1} g_{2} e_{1} e_{3} g_{2} g_{1}\right)=g_{3} f_{k} \text {. }
$$

Consider $k>2$. If $2 \leq i<k$, then, using Lemma 3.9, we have

$$
\begin{aligned}
g_{i} f_{k} & =g_{i}\left(g_{1} g_{2} \cdots g_{2 k-2}\right) f_{k-1} e_{2 k-1}\left(g_{2 k-2} \cdots g_{2} g_{1}\right) \\
& =\left(g_{1} g_{2} \cdots g_{2 k-2}\right) g_{i-1} f_{k-1} e_{2 k-1}\left(g_{2 k-2} \cdots g_{2} g_{1}\right) \\
& =\left(g_{1} g_{2} \cdots g_{2 k-2}\right) g_{2 k-i-1} f_{k-1} e_{2 k-1}\left(g_{2 k-2} \cdots g_{2} g_{1}\right),
\end{aligned}
$$

which by the induction hypothesis equals

$$
g_{2 k-i}\left(g_{1} g_{2} \cdots g_{2 k-2}\right) f_{k-1} e_{2 k-1}\left(g_{2 k-2} \cdots g_{2} g_{1}\right)=g_{2 k-i} f_{k} .
$$

The last case to check is $k>2$ and $i=1$. We have

$$
\begin{aligned}
g_{1} f_{k}= & g_{1}\left(g_{1} g_{2} \cdots g_{2 k-2}\right) f_{k-1} e_{2 k-1}\left(g_{2 k-2} \cdots g_{2} g_{1}\right) \\
= & g_{1}\left(g_{1} g_{2} \cdots g_{2 k-2}\right)\left(\left(g_{1} g_{2} \cdots g_{2 k-4}\right) f_{k-2} e_{2 k-3}\left(g_{2 k-4} \cdots g_{2} g_{1}\right)\right) \\
& \cdot e_{2 k-1}\left(g_{2 k-2} \cdots g_{2} g_{1}\right) \\
= & g_{1}\left(g_{2} g_{3} \cdots g_{2 k-3}\right)\left(g_{1} g_{2} \cdots g_{2 k-2}\right) f_{k-2} e_{2 k-3}\left(g_{2 k-4} \cdots g_{2} g_{1}\right) \\
& \cdot e_{2 k-1}\left(g_{2 k-2} \cdots g_{2} g_{1}\right),
\end{aligned}
$$

by repeated use of Lemma 3.9(4). Moving $e_{2 k-3}$ and $e_{2 k-1}$ to the left and applying Lemma $3.9(3)$, we get

$$
\begin{gathered}
g_{1}\left(g_{2} g_{3} \cdots g_{2 k-3}\right)\left(g_{1} g_{2} \cdots g_{2 k-4}\left(g_{2 k-3} g_{2 k-2} e_{2 k-3} e_{2 k-1}\right)\right) \\
\cdot f_{k-2}\left(g_{2 k-4} \cdots g_{2} g_{1}\right)\left(g_{2 k-2} \cdots g_{2} g_{1}\right) \\
=g_{1}\left(g_{2} g_{3} \cdots g_{2 k-3}\right)\left(g_{1} g_{2} \cdots g_{2 k-4}\left(g_{2 k-1} g_{2 k-2} e_{2 k-3} e_{2 k-1}\right)\right) \\
\cdot f_{k-2}\left(g_{2 k-4} \cdots g_{2} g_{1}\right)\left(g_{2 k-2} \cdots g_{2} g_{1}\right) .
\end{gathered}
$$

Now $g_{2 k-1}$ and $g_{2 k-2}$ can be moved to the left and $e_{2 k-1}$ and $e_{2 k-3}$ to the right to yield

$$
\begin{array}{r}
g_{2 k-1} g_{1}\left(g_{2} g_{3} \cdots g_{2 k-3} g_{2 k-2}\right)\left(g_{1} g_{2} \cdots g_{2 k-4}\right) f_{k-2}\left(e_{2 k-3} e_{2 k-1}\right) \\
\cdot\left(g_{2 k-4} \cdots g_{2} g_{1}\right)\left(g_{2 k-2} \cdots g_{2} g_{1}\right) \\
=g_{2 k-1} f_{k} .
\end{array}
$$

This completes the proof of (1). 
Statement (2) is also proved by induction on $k$. For $k=2$ and $i=1$, we have

$$
e_{1} f_{2}=e_{1} e_{2} e_{1} e_{3} e_{2}=e_{1} e_{3} e_{2},
$$

and similarly $e_{3} f_{2}=e_{1} e_{3} e_{2}$.

For $2 \leq i<k$, exactly the same induction step can be used as in the proof of statement (1). The only remaining case to check is $k>2$ and $i=1$.

We claim that $e_{1} f_{k}$ is the word $w_{k}$ in the $e_{i}$ 's obtained by deleting the two columns to the left of the middle column in the diamond expression for $f_{k}$. For example,

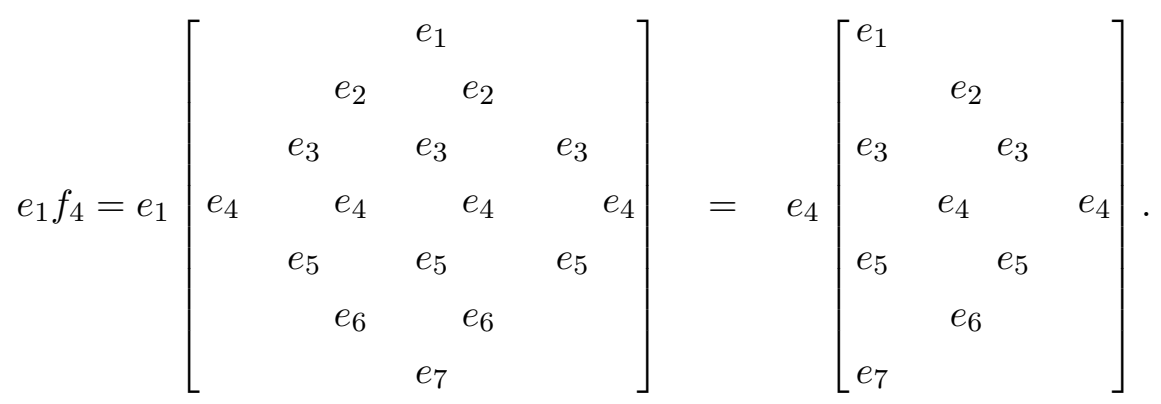

This claim can be proved by induction on $k$. Moreover, both $f_{k}$ and $w_{k}$ are invariant under the automorphism $\varrho_{2 k}$ of $W_{2 k}$. Applying this automorphism to the equation $e_{1} f_{k}=w_{k}$ gives

$$
e_{2 k-1} f_{k}=w_{k}=e_{1} f_{k} .
$$

This completes the proof of (2).

Statements (3) and (4) follow from (1) and (2) by applying the shift homomorphism $S$, for example

$$
e_{i} S\left(f_{k}\right)=S\left(e_{i-1} f_{k}\right)=S\left(e_{2 k+1-i} f_{k}\right)=e_{2 k+2-i} S\left(f_{k}\right) .
$$

REMARK 5.27. One can give a similar explicit expression for $e_{i} f_{k}$ for all $i<k$. One obtains $e_{k-1} f_{k}$ by deleting the leftmost $e_{k}$ from the diamond expression for $f_{k}$. Moreover, if $i<k-1$, then $e_{i} f_{k}$ is the word in the $e_{j}$ 's obtained by deleting the two columns just to the left of the first column beginning with $e_{i}$ in the diamond expression for $f_{k}$. For example,

$$
e_{2} f_{4}=\left[\begin{array}{ccccc}
e_{2} & & e_{1} & & \\
& e_{3} & & e_{3} \\
e_{4} & & e_{4} & & e_{4} \\
& e_{5} & & e_{5} \\
e_{6} & & e_{6} & & \\
& e_{7} & &
\end{array}\right] .
$$


The proof of statement (2) in the previous proposition can be based entirely on this observation.

\section{Proposition 5.28.}

(1) For all $k, W_{2 k} f_{k}=W_{k} f_{k}$ and $f_{k} W_{2 k}=f_{k} W_{k}$.

(2) For all $k, W_{2 k+1} S\left(f_{k}\right)=W_{k+1} S\left(f_{k}\right)$ and $S\left(f_{k}\right) W_{2 k+1}=S\left(f_{k}\right) W_{k+1}$.

Proof. The second part of each statement follows from the first part by applying the symmetry $\alpha$.

To prove (1), it suffices to show that $W_{k} f_{k}$ is a left ideal in $W_{2 k}$. For this, it is enough to show that $e_{i} W_{k} f_{k} \subseteq W_{k} f_{k}$ and $g_{i}^{ \pm 1} W_{k} f_{k} \subseteq W_{k} f_{k}$ for $k \leq i \leq 2 k-1$. For $k<i<2 k-1$, this follows from Proposition 5.26, since $e_{i}$ and $g_{i}^{ \pm 1}$ commute with $W_{k}$. Finally, by Proposition 5.24 , we can write $f_{k}=e_{k} r_{k}$ for some $r_{k} \in W_{2 k}$. Hence for $\chi \in\left\{e_{k}, g_{k}^{ \pm 1}\right\}$,

$$
\chi W_{k} f_{k}=\chi W_{k} e_{k} r_{k} \subseteq W_{k+1} e_{k} r_{k}=W_{k} e_{k} r_{k}=W_{k} f_{k},
$$

using Corollary 3.21. The proof of part (2) is similar.

Definition 5.29. Let $n \geq 2 k$ and put $r=n-2 k$. Define $W_{n}^{(r)}$ to be the ideal in $W_{n}$ generated by $e_{1} e_{3} \cdots e_{2 k-1}$.

Evidently, one has

$$
\begin{array}{ll}
W_{n}^{(0)} \subseteq W_{n}^{(2)} \subseteq \cdots \subseteq W_{n}^{(n)}=W_{n} & \text { if } n \text { is even, } \\
W_{n}^{(1)} \subseteq W_{n}^{(3)} \subseteq \cdots \subseteq W_{n}^{(n)}=W_{n} & \text { if } n \text { is odd. }
\end{array}
$$

Morton and Wassermann show that $\varphi\left(W_{n}^{(r)}\right)$ is the ideal $K T_{n}^{(r)}$ in $\mathrm{KT}_{n}$ spanned by tangle diagrams of rank no more than $r$, that is, tangle diagrams of the form $S T$, where $T$ is an $(n, r)$-tangle diagram and $S$ is an $(r, n)$-tangle diagram. We will not need this observation, but it provides the motivation for the definition of $W_{n}^{(r)}$.

Lemma 5.30. Let $n \geq 2 k$ and put $r=n-2 k$.

(1) $W_{n}^{(r)}$ is the ideal in $W_{n}$ generated by $f_{k}$.

(2) If $n>2 k$ then $W_{n}^{(r)}$ is the ideal in $W_{n}$ generated by $S\left(f_{k}\right)$.

Proof. It follows from the definition of $f_{k}$, and induction on $k$, that $f_{k}=a\left(e_{1} e_{3} \cdots e_{2 k-1}\right) b$ where $a$ and $b$ are in the monoid generated by $\left\{g_{j}\right\}$, and in particular are invertible. Likewise, if $n>2 k$, then $S\left(f_{k}\right)=\operatorname{Ad}(a)\left(f_{k}\right)$ where $a$ is invertible.

Corollary 5.31.

(1) $W_{2 k}^{(0)}=W_{k} f_{k} W_{k}$.

(2) $W_{2 k+1}^{(1)}=W_{k+1} S\left(f_{k}\right) W_{k+1}$.

Proof. Immediate from Proposition 5.28 and Lemma 5.30. 
5.5. Injectivity of $\varphi: W_{n} \rightarrow \mathrm{KT}_{n}$. Fix a ring $R$ and write $W_{n}$ for $W_{n, R}$ and $\mathrm{KT}_{n}$ for $\mathrm{KT}_{n, R}$. This section contains Morton and Wassermann's proof of the injectivity of $\varphi: W_{n} \rightarrow \mathrm{KT}_{n}$. The strategy is to show that $\varphi$ is injective on $W_{n}^{(r)}$ for all $n$ and $r$, by double induction.

Proposition 5.32. Suppose $\left.\varphi\right|_{W_{n-1}}$ is injective for some $n$. Then $\left.\varphi\right|_{W_{n}^{(0)}}$ is injective if $n$ is even, and $\left.\varphi\right|_{W_{n}^{(1)}}$ is injective if $n$ is odd.

Proof. Consider the case that $n=2 k$. By Corollary 5.31, $W_{n}^{(0)}=$ $W_{k} f_{k} W_{k}$. By Theorem 5.8, $\mathrm{KT}_{k}$ has a basis $\left\{T_{c}: c\right.$ a $k$-connector $\}$ consisting of totally descending tangle diagrams with distinct connectors. Since by hypothesis, $\varphi: W_{k} \rightarrow \mathrm{KT}_{k}$ is an isomorphism, it follows that $\left\{\varphi^{-1}\left(T_{c}\right)\right.$ : $c$ a $k$-connector $\}$ is a basis of $W_{k}$. Therefore $W_{n}^{(0)}$ is spanned by the set

$$
\left\{\varphi^{-1}\left(T_{c}\right) f_{k} \varphi^{-1}\left(T_{d}\right): c, d k \text {-connectors }\right\} \text {. }
$$

The image of this spanning set under $\varphi$ is $\left\{T_{c} F_{k} T_{d}: c, d k\right.$-connectors $\}$. This set of $(n, n)$-tangle diagrams has distinct connectors, and therefore is linearly independent by Corollary 4.8. It follows that $\varphi$ is injective on $W_{n}^{(0)}$. The proof for the case $n=2 k+1$ is similar.

Next we introduce a linear complement of $W_{n}^{(r-2)}$ in $W_{n}^{(r)}$.

Definition 5.33. Let $n>2 k$ and put $r=n-2 k$. Then $V_{n}^{(r)}$ is the span of $\left\{g_{\pi}\left[w \otimes g_{\tau}\right] \alpha\left(g_{\sigma}\right)\right\}$, where $w \in W_{2 k}^{(0)}, \pi$ and $\sigma$ are $(2 k, r)$-shuffles, $\tau$ is an $r$-permutation, and $g_{\pi}, g_{\sigma}$, and $g_{\tau}$ are the corresponding positive permutation braids.

Lemma 5.34. Assume that $\left.\varphi\right|_{W_{n-1}}$ is injective for a particular $n$.

(1) $\left.\varphi\right|_{V_{n}^{(r)}}$ is injective for $r>0$.

(2) $V_{n}^{(r)} \cap W_{n}^{(r-2)}=(0)$.

Proof. By assumption, $\left.\varphi\right|_{W_{2 k}^{(0)}}$ is injective, and moreover, $W_{2 k}^{(0)}$ has a basis consisting of elements $\varphi^{-1}\left(T_{c}\right) f_{k} \varphi^{-1}\left(T_{d}\right)$, where $T_{c}$ and $T_{d}$ vary independently over a set of totally descending $(k, k)$-tangle diagrams with distinct connectors. Thus $V_{n}^{(r)}$ has a spanning set

$$
\left\{g_{\pi}\left[\varphi^{-1}\left(T_{c}\right) f_{k} \varphi^{-1}\left(T_{d}\right) \otimes g_{\tau}\right] \alpha\left(g_{\sigma}\right)\right\} .
$$

The image of this set,

$$
\left\{G_{\pi}\left[T_{c} F_{k} T_{d} \otimes G_{\tau}\right] \alpha\left(G_{\sigma}\right)\right\},
$$

is a family of tangle diagrams with distinct connectors $\pi\left[c F_{k} d \otimes \tau\right] \sigma^{-1}$, and is therefore linearly independent by Corollary 4.8. This proves statement (1).

For (2), $\varphi\left(W_{n}^{(r-2)}\right)$ is spanned by totally descending tangle diagrams with no more than $r-2$ through strands (i.e. strands connecting top to 
bottom), while $\varphi\left(V_{n}^{(r)}\right)$ is spanned by totally descending tangle diagrams with exactly $r$ through strands. By Corollary 4.8, $\varphi\left(V_{n}^{(r)}\right) \cap \varphi\left(W_{n}^{(r-2)}\right)=(0)$, so also $V_{n}^{(r)} \cap W_{n}^{(r-2)}=(0)$.

Corollary 5.35. If $\varphi$ is injective on $W_{n-1}$ and also on $W_{n}^{(r-2)}$, then $\varphi$ is injective on $W_{n}^{(r-2)} \oplus V_{n}^{(r)}$.

The remainder of the proof consists of showing that $W_{n}^{(r-2)} \oplus V_{n}^{(r)}=$ $W_{n}^{(r)}$. Since $e_{1} e_{3} \cdots e_{2 k-1} \in V_{n}^{(r)}$, it suffices to show that $W_{n}^{(r-2)} \oplus V_{n}^{(r)}$ is an ideal in $W_{n}$. Because of the invariance of $W_{n}^{(r-2)} \oplus V_{n}^{(r)}$ under the anti-automorphism $\alpha$, it is enough to show that $W_{n}^{(r-2)} \oplus V_{n}^{(r)}$ is a left ideal. It suffices to show that $\chi V_{n}^{(r)} \subseteq W_{n}^{(r-2)} \oplus V_{n}^{(r)}$ for $\chi$ a generator of $W_{n}$, because $W_{n}^{(r-2)}$ is already an ideal. Moreover, since $g_{i}^{-1}=g_{i}-z e_{i}+z$, it suffices to show this for $\chi \in\left\{e_{i}, g_{i}: 1 \leq i \leq n-1\right\}$.

Note that $V_{n}^{(n)}$ is the span of $\left\{g_{\pi}: \pi \in \mathfrak{S}_{n}\right\}$ and $W_{n}^{(n-2)}=W_{n} e_{1} W_{n}$, so a particular case of our assertion is that $V_{n}^{(n)} \oplus W_{n} e_{1} W_{n}=W_{n}$.

LEMMA 5.36. Let $n>2 k$ and $r=n-2 k$.

(1) $V_{n}^{(n)} \oplus W_{n} e_{1} W_{n}=W_{n}$.

(2) $W_{2 k}^{(0)} \otimes W_{r} \subseteq W_{n}^{(r-2)}+W_{2 k}^{(0)} \otimes V_{r}^{(r)} \subseteq W_{n}^{(r-2)}+V_{n}^{(r)}$.

(3) $g_{\pi}\left(W_{2 k}^{(0)} \otimes W_{r}\right) g_{\sigma} \subseteq W_{n}^{(r-2)}+V_{n}^{(r)}$ for any positive permutation braids $g_{\pi}, g_{\sigma}$.

Proof. For (1), we have to show that $\chi g_{\pi} \in V_{n}^{(n)}+W_{n} e_{1} W_{n}$ for $\chi \in$ $\left\{e_{i}, g_{i}: 1 \leq i \leq n-1\right\}$. We have $e_{i} g_{\pi} \in W_{n} e_{i} W_{n}=W_{n} e_{1} W_{n}$ for all $i$. If $\ell\left(s_{i} \pi\right)=\ell(\pi)+1$, then $g_{i} g_{\pi}=g_{s_{i} \pi} \in V_{n}^{(n)}$; on the other hand, if $\ell\left(s_{i} \pi_{i}\right)=$ $\ell(\pi)-1$, then $g_{\pi}=g_{i} g_{s_{i} \pi}$, and

$$
g_{i} g_{\pi}=\left(g_{i}^{2}\right) g_{s_{i} \pi}=g_{s_{i} \pi}-z g_{\pi}+z \lambda^{-1} e_{i} g_{s_{i} \pi} \in V_{n}^{(n)}+W_{n} e_{1} W_{n} .
$$

Applying (1) to $W_{r}$, we get $W_{r}=W_{r} e_{1} W_{r}+V_{r}^{(r)}$, so

$$
W_{2 k}^{(0)} \otimes W_{r}=W_{2 k}^{(0)} \otimes W_{r} e_{1} W_{r}+W_{2 k}^{(0)} \otimes V_{r}^{(r)} \subseteq W_{n}^{(r-2)}+V_{n}^{(r)} .
$$

This shows (2).

Using Lemma 5.17, the positive permutation braid $g_{\pi}$ can be written as $g_{\pi_{1}} w_{1}$, where $\pi_{1}$ is a $(2 k, r)$-shuffle and $w_{1} \in W_{2 k} \otimes W_{r}$. Applying the same result to $\alpha\left(g_{\sigma}\right)$, we get $g_{\sigma}=w_{2} \alpha\left(g_{\sigma_{1}}\right)$, where $\sigma_{1}$ is a $(2 k, r)$-shuffle and $w_{2} \in W_{2 k} \otimes W_{r}$. Thus

$$
g_{\pi}\left(W_{2 k}^{(0)} \otimes W_{r}\right) g_{\sigma} \subseteq g_{\pi_{1}}\left(W_{2 k}^{(0)} \otimes W_{r}\right) \alpha\left(g_{\sigma_{1}}\right) .
$$

By statement (2), this lies in

$$
W_{n}^{(r-2)}+g_{\pi_{1}}\left(W_{2 k}^{(0)} \otimes V_{r}^{(r)}\right) \alpha\left(g_{\sigma_{1}}\right) \subseteq W_{n}^{(r-2)}+V_{n}^{(r)} .
$$


LEMma 5.37. $e_{i} V_{n}^{(r)} \subseteq W_{n}^{(r-2)}+V_{n}^{(r)}$ and $g_{i} V_{n}^{(r)} \subseteq W_{n}^{(r-2)}+V_{n}^{(r)}$ for $1 \leq i \leq n-1$.

Proof. Consider $x=g_{\pi}\left[w \otimes g_{\tau}\right] \alpha\left(g_{\sigma}\right)$, where $\pi$ and $\sigma$ are $(2 k, r)$-shuffles, $w \in W_{2 k}^{(0)}$, and $\tau \in \mathfrak{S}_{r}$. We have to show that $e_{i} x$ and $g_{i} x$ lie in $W_{n}^{(r-2)}+V_{n}^{(r)}$ for all $1 \leq i \leq n-1$.

Suppose that $\pi^{-1}(i)>\pi^{-1}(i+1)$. Then $g_{\pi}=g_{i} g_{\pi_{1}}$, where $\pi_{1}{ }^{-1}(i)<$ $\pi_{1}^{-1}(i+1)$, and $\pi_{1}$ is also a $(2 k, r)$-shuffle. Thus $e_{i} g_{\pi}=e_{i} g_{i} g_{\pi_{1}}=\lambda^{-1} e_{i} g_{\pi_{1}}$. Likewise, $g_{i} g_{\pi}=\left(g_{i}\right)^{2} g_{\pi_{1}}=g_{\pi_{1}}-z g_{\pi}+z \lambda^{-1} e_{i} g_{\pi_{1}}$. We are therefore reduced to considering the case that $\pi^{-1}(i)<\pi^{-1}(i+1)$.

If $\pi^{-1}(i)<\pi^{-1}(i+1)$, then

$$
g_{i} x=g_{s_{i} \pi}\left[w \otimes g_{\tau}\right] \alpha\left(g_{\sigma}\right) \subseteq W_{n}^{(r-2)}+V_{n}^{(r)},
$$

by Lemma 5.36 .

It remains to consider $e_{i} x$ when $\pi^{-1}(i)<\pi^{-1}(i+1)$.

If $\pi^{-1}(i+1) \leq 2 k$ or $\pi^{-1}(i) \geq 2 k+1$, then $\pi^{-1}(i+1)=\pi^{-1}(i)+1$. By Lemmas 5.18 and 5.36,

$$
e_{i} x=g_{\pi} e_{\pi^{-1}(i)}\left[w \otimes g_{\tau}\right] \alpha\left(g_{\sigma}\right) \in g_{\pi}\left[W_{2 k}^{(0)} \otimes W_{r}\right] \alpha\left(g_{\sigma}\right) \subseteq W_{n}^{(r-2)}+V_{n}^{(r)} .
$$

The remaining case to consider is $\pi^{-1}(i) \leq 2 k$ and $\pi^{-1}(i+1) \geq 2 k+1$. Define a permutation $\varrho$ by

$$
\varrho(j)= \begin{cases}j & \text { if } j<\pi^{-1}(i), \\ j+1 & \text { if } \pi^{-1}(i) \leq j<2 k, \\ \pi^{-1}(i) & \text { if } j=2 k, \\ \pi^{-1}(i+1) & \text { if } j=2 k+1, \\ j-1 & \text { if } 2 k+1<j \leq \pi^{-1}(i+1), \\ j & \text { if } j>\pi^{-1}(i+1) .\end{cases}
$$

Since $\varrho \in \mathfrak{S}_{2 k} \times \mathfrak{S}_{r}, \ell(\pi \varrho)=\ell(\pi)+\ell(\varrho)$ and $g_{\pi \varrho}=g_{\pi} g_{\varrho}$. The permutation $\pi \varrho$ has the following properties: $\pi \varrho(2 k)=i ; \pi \varrho(2 k+1)=i+1$; if $1 \leq a<$ $b \leq 2 k-1$ or $2 k+2 \leq a<b \leq n$, then $\pi \varrho(a)<\pi \varrho(b)$. We have

$$
e_{i} g_{\pi}=e_{i} g_{\pi} g_{\varrho} g_{\varrho}^{-1}=e_{i} g_{\pi \varrho} g_{\varrho}^{-1}=g_{\pi \varrho} e_{2 k} g_{\varrho}^{-1} \text {. }
$$

Therefore

$$
e_{i} x \in g_{\pi \varrho} e_{2 k}\left(W_{2 k}^{(0)} \otimes W_{r}\right) \alpha\left(g_{\sigma}\right) .
$$

We concentrate on $e_{2 k}\left(W_{2 k}^{(0)} \otimes W_{r}\right)$.

Lemma 5.38. Suppose $\varphi$ is injective on $W_{2 k}^{(0)}$. Then $W_{2 k}^{(0)}$ is spanned by elements of the form

$$
g_{m} g_{m+1} \cdots g_{2 k-2} e_{2 k-1} w,
$$

where $1 \leq m \leq 2 k-2$ and $w \in W_{2 k}^{(0)}$. 
Proof. By assumption, $\varphi$ is an isomorphism from $W_{2 k}^{(0)}$ to the ideal $K T_{2 k}^{(0)}$ of $\mathrm{KT}_{2 k}$ spanned by tangle diagrams of rank 0 . Now, $K T_{2 k}^{(0)}$ is spanned by totally descending tangle diagrams, and we can choose the order of the strands so that the strand incident with the vertex $\overline{2 k}$ lies above all other strands. Such a totally descending tangle diagram is isotopic to a tangle diagram of the following form:

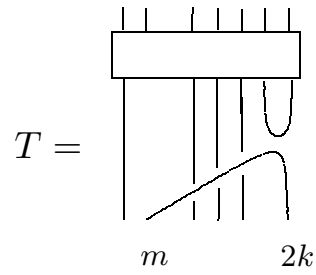

Such a tangle diagram has the factorization $T=G_{m} \cdots G_{2 k-2} E_{2 k-1} T^{\prime}$.

LEMMA 5.39. $g_{\pi \varrho} e_{2 k} g_{m} g_{m+1} \cdots g_{2 k-2} e_{2 k-1}=g_{\pi^{\prime}} e_{2 k-1}$ for some positive permutation braid $g_{\pi^{\prime}}$.

Proof. First, $e_{2 k}$ can be moved to the right of $g_{m} g_{m+1} \cdots g_{2 k-2}$ and $e_{2 k} e_{2 k-1}$ can be written as $g_{2 k-1} g_{2 k} e_{2 k-1}$ or as $g_{2 k-1}^{-1} g_{2 k}^{-1} e_{2 k-1}$. Therefore

$$
\begin{aligned}
g_{\pi \varrho} e_{2 k} g_{m} g_{m+1} \cdots g_{2 k-2} e_{2 k-1} & =g_{\pi \varrho}\left(g_{m} g_{m+1} \cdots g_{2 k-2}\right)\left(g_{2 k-1} g_{2 k}\right) e_{2 k-1} \\
& =g_{\pi \varrho}\left(g_{m} g_{m+1} \cdots g_{2 k-2}\right)\left(g_{2 k-1}^{-1} g_{2 k}^{-1}\right) e_{2 k-1} .
\end{aligned}
$$

Since $\pi \varrho(a)<\pi \varrho(b)$ if $1 \leq a<b \leq 2 k-1, g_{\pi \varrho} g_{m} g_{m+1} \cdots g_{2 k-2}$ is the positive permutation braid corresponding to the permutation $\pi \varrho s_{m} \cdots s_{2 k-2}$. Moreover,

$$
\begin{aligned}
\pi \varrho s_{m} \ldots s_{2 k-2}(2 k-1) & =\pi \varrho(m), \\
\pi \varrho s_{m} \ldots s_{2 k-2}(2 k) & =\pi \varrho(2 k)=i, \\
\pi \varrho s_{m} \ldots s_{2 k-2}(2 k+1) & =\pi \varrho(2 k+1)=i+1 .
\end{aligned}
$$

It follows that if $\pi \varrho(m)<i$, then $g_{\pi \varrho}\left(g_{m} g_{m+1} \cdots g_{2 k-2}\right)\left(g_{2 k-1} g_{2 k}\right)$ is a positive permutation braid, while if $\pi \varrho(m)>i+1$, then $g_{\pi \varrho}\left(g_{m} g_{m+1} \cdots g_{2 k-2}\right)$. $\left(g_{2 k-1}^{-1} g_{2 k}^{-1}\right)$ is a positive permutation braid. In either case, the desired conclusion follows.

End of the proof of Lemma 5.37. We have to show that

$$
g_{\pi \varrho} e_{2 k}\left(W_{2 k}^{(0)} \otimes W_{r}\right) \alpha\left(g_{\sigma}\right) \subseteq W_{n}^{(r-2)}+V_{n}^{(r)} .
$$

By Lemma 5.38, it is enough to show

$$
g_{\pi \varrho} e_{2 k}\left(g_{m} \cdots g_{2 k-2}\right) e_{2 k-1}\left(W_{2 k}^{(0)} \otimes W_{r}\right) \alpha\left(g_{\sigma}\right) \subseteq W_{n}^{(r-2)}+V_{n}^{(r)} .
$$

But by Lemmas 5.39 and 5.36 ,

$$
\begin{aligned}
& g_{\pi \varrho} e_{2 k}\left(g_{m} \cdots g_{2 k-2}\right) e_{2 k-1}\left(W_{2 k}^{(0)} \otimes W_{r}\right) \alpha\left(g_{\sigma}\right) \\
& =g_{\pi^{\prime}} e_{2 k-1}\left(W_{2 k}^{(0)} \otimes W_{r}\right) \alpha\left(g_{\sigma}\right) \subseteq W_{n}^{(r-2)}+V_{n}^{(r)} .
\end{aligned}
$$


Proposition 5.40. If $\varphi$ is injective on $W_{n-1}$ then

$$
V_{n}^{(r)} \oplus W_{n}^{(r-2)}=W_{n}^{(r)} .
$$

Proof. We have shown that $V_{n}^{(r)} \oplus W_{n}^{(r-2)}$ is an ideal containing $e_{1} \cdots e_{2 k-1}$. Therefore $V_{n}^{(r)} \oplus W_{n}^{(r-2)}=W_{n}^{(r)}$.

TheOREM 5.41. For all $n, \varphi: W_{n} \rightarrow \mathrm{KT}_{n}$ is an isomorphism.

Proof. Surjectivity was shown in Theorem 5.20. Injectivity is evident for $n=0,1$. We show $\varphi$ is injective on all ideals $W_{n}^{(r)}$ by double induction on $n$ and $r$. By Proposition 5.32, if $\varphi$ is injective on $W_{n-1}$, then $\varphi$ is injective on $W_{n}^{(0)}$ (if $n$ is even) or on $W_{n}^{(1)}$ (if $n$ is odd). By Corollary 5.35 and Proposition 5.40 , if $\varphi$ is injective on $W_{n-1}$ and on $W_{n}^{(r-2)}$, then $\varphi$ is injective on $W_{n}^{(r)}$.

Corollary 5.42. For all $R$ and $n, W_{n, R}$ is a free $R$-module with basis $\left\{\varphi^{-1}\left(T_{c}\right): c\right.$ is an n-connector $\}$.

Corollary 5.43. For all $R$ and $n, W_{n, R} \cong W_{n, \Lambda} \otimes_{\Lambda} R$.

Proof. $W_{n, R} \cong \mathrm{KT}_{n, R}$ and $W_{n, \Lambda} \cong \mathrm{KT}_{n, \Lambda}$. But $\mathrm{KT}_{n, R} \cong \mathrm{KT}_{n, \Lambda} \otimes_{\Lambda} R$, by Corollary 5.9 .

COROLlaRY 5.44. If $R$ is a ring with distinguished elements $\lambda, z$ and $\delta$, and $S \supseteq R$ is a ring containing $R$, then $W_{n, R}$ imbeds in $W_{n, S}$.

COROLlary 5.45. If $S$ is a ring with distinguished elements $\lambda, z, \delta$, and $q_{r}(r \geq 1)$, then $i_{n}: W_{n, S} \rightarrow \widehat{W}_{n, S}$ is injective.

Proof. Consider the commuting diagram

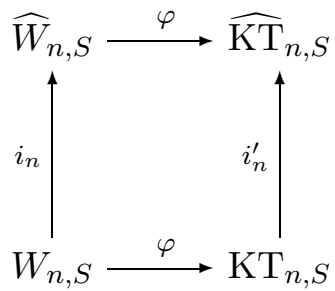

Since $\varphi: W_{n, S} \rightarrow \mathrm{KT}_{n, S}$ is an isomorphism, $i_{n}^{\prime}=\varphi \circ i_{n} \circ \varphi^{-1}$. But $i_{n}^{\prime}$ is injective by Remark 2.11, and therefore $i_{n}$ is injective as well.

COROllary 5.46. For all $R$ and $n$,

(1) There is a conditional expectation $\varepsilon_{n}: W_{n, R} \rightarrow W_{n-1, R}$ such that $e_{n} x e_{n}=\delta \varepsilon_{n}(x)$ for $x \in W_{n, R}$.

(2) The map $\iota: W_{n-1, R} \rightarrow W_{n, R}$ is injective (and $\varepsilon_{n} \circ \iota(x)=x$ for $\left.x \in W_{n-1, R}\right)$.

(3) There is a trace $\varepsilon: W_{n, R} \rightarrow R$ such that $\varepsilon \circ \varepsilon_{n+1}(x)=\varepsilon(x)$ for $x \in W_{n+1, R}$ and $\varepsilon \circ \iota(x)=\varepsilon(x)$ for $x \in W_{n-1, R}$. 
Proof. This follows from the isomorphism $W_{n, R} \cong \mathrm{KT}_{n, R}$ and the corresponding properties of $\mathrm{KT}_{n, R}$.

\section{ISOMORPHISM OF AFFINE BMW AND KAUFFMAN TANGLE ALGEBRAS}

This section contains our main result, the isomorphism of $\widehat{W}_{n, S}$ and $\widehat{\mathrm{KT}}_{n, S}$ for any ring $S$ with distinguished elements $\lambda, z, \delta$, and $q_{r}(r \geq 1)$. We also give a basis of $\widehat{\mathrm{KT}}_{n, S}$ over $S$.

6.1. Surjectivity of $\varphi: \widehat{W}_{n} \rightarrow \widehat{\mathrm{KT}}_{n}$. Fix a ring $S$ with distinguished elements $\lambda, z, \delta$, and $q_{r}(r \geq 1)$.

Definition 6.1. A simple winding is a piece of an affine tangle diagram with one ordinary strand, without self-crossings, regularly isotopic to the intersection of one of the affine tangle diagrams $X_{1}$ or $X_{1}^{-1}$ with a small neighborhood of the flagpole, as in the following figure:

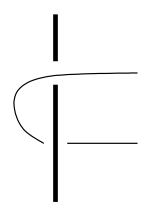

Definition 6.2. An affine tangle diagram is in standard position if:

(1) It has no crossings to the left of the flagpole.

(2) There is a neighborhood of the flagpole whose intersection with the tangle diagram is a union of simple windings.

(3) The simple windings have no crossings and are not nested. That is, between the two crossings of a simple winding with the flagpole, there is no other crossing of a strand with the flagpole.

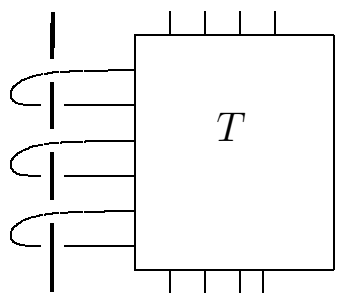

Lemma 6.3. Any affine tangle diagram is regularly isotopic to an affine tangle diagram in standard position. 
Proof. An affine $(n, n)$-tangle can be viewed as the union of

(1) an ordinary tangle diagram $T_{1}$ with $2 n+2 l$ boundary points to the right of the flagpole,

(2) an ordinary tangle diagram $T_{2}$ with $2 l$ boundary points to the left of the flagpole, and

(3) $2 l$ horizontal strands connecting $T_{1}$ and $T_{2}$ and crossing above or below the flagpole.

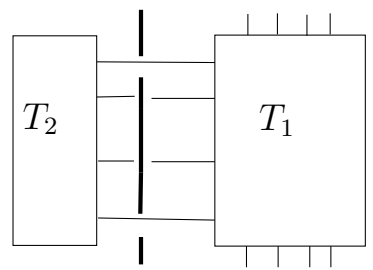

From each horizontal strand crossing over the flagpole, pull a finger over the tangle diagram $T_{2}$.

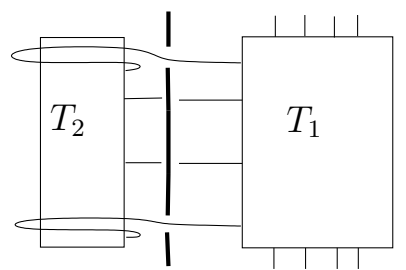

Now slide $T_{1}$ and $T_{2}$ to the right, with the diagram $T_{2}$ sliding under the flagpole. The result is a diagram in standard position.

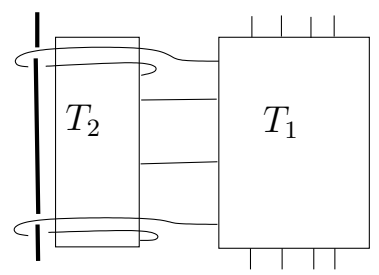

We give each affine tangle diagram in standard position a standard orientation (see Section 5.1).

In the oriented diagram, the simple windings are of the four possible types pictured below:

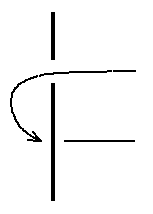

(a)

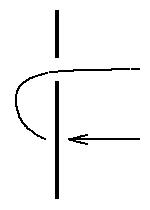

(b)

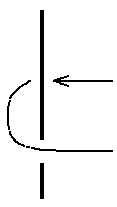

(c)

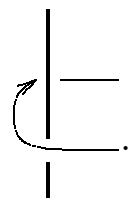

(d) 
Lemma 6.4. Let $T$ be an affine tangle diagram in standard position and with a standard orientation. Suppose $T$ has $l$ simple windings and $K$ crossings of ordinary strands. If $T$ has a simple winding on a non-closed strand, then $T$ can be written as $T=A T_{0}+B$ or $T=T_{0} A+B$, where $A$ is in the monoid generated by $\left\{X_{1}^{ \pm 1}, G_{i}^{ \pm 1}\right\}, T_{0}$ is an affine tangle diagram in standard position with $l-1$ simple windings, and $B$ is in the span of affine tangle diagrams in standard position with fewer than $K$ crossings of ordinary strands and at most $l$ simple windings.

Proof. Let $s$ be a non-closed strand of $T$ with a simple winding. Suppose that the initial endpoint of $s$ is on the top boundary of $R$, say the $j$ th vertex on the top boundary. (Otherwise, the final endpoint of $s$ is on the bottom boundary of $R$, and this case can be handled similarly.) Consider the first simple winding on $s$.

If the simple winding is of type (a) or (d), then consider the affine tangle diagram $T^{\prime}$ obtained from $T$ by reversing crossings of $s$ as necessary so that all crossings of $s$ with other ordinary strands are over-crossings, and all self-crossings of $s$ are encountered first as over-crossings. By the Kauffman skein relation, $T$ and $T^{\prime}$ are congruent modulo the span of affine tangle diagrams in standard position with fewer than $K$ crossings (and at most $l$ simple windings). Consider the diagram $T_{0}=T^{\prime} G_{j}^{-1} \cdots G_{1}^{-1} X_{1}^{-1}$. It has two simple windings, one of type (c) and the other of type (a) or (d), connected by an unknotted arc which has only over-crossings with other arcs; thus $T_{0}$ is regularly isotopic to an affine tangle diagram in standard position with $l-1$ simple windings:

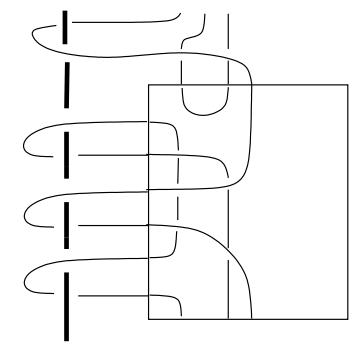

We have $T=T^{\prime}+B=T_{0} X_{1} G_{1} \cdots G_{j}+B$, where $B$ is in the span of affine tangle diagrams in standard position with fewer than $K$ crossings and at most $\ell$ simple windings.

If the first simple winding on $s$ is of type (b) or (c), then take $T^{\prime}$ to be the diagram obtained from $T$ by changing crossings of $s$ so that all crossings of $s$ with other ordinary strands are under-crossings, and all self-crossings of $s$ are encountered first as under-crossings, and take $T_{0}=T^{\prime} G_{j} \cdots G_{1} X_{1}$. Now $T_{0}$ has two simple windings, one of type (a) and the other of type (b) or (c), connected by an unknotted arc which has only under-crossings with other 
arcs; again, $T_{0}$ is regularly isotopic to an affine tangle diagram in standard position with $l-1$ simple windings.

Say that the oriented affine tangle diagram $T$ is totally descending if, as $T$ is traversed in accordance with the orientation, each crossing of ordinary strands is encountered the first time as an over-crossing. By the inductive argument of Proposition 5.5, $\widehat{\mathrm{KT}}_{n}$ is spanned by totally descending affine tangle diagrams in standard position.

TheOREM 6.5. $\varphi: \widehat{W}_{n, S} \rightarrow \widehat{\mathrm{KT}}_{n, S}$ is surjective.

Proof. We have to show that each totally descending oriented affine tangle diagram $T$ in standard position is in the subalgebra of $\widehat{\mathrm{KT}}_{n}$ generated by $X_{1}^{ \pm 1}$ and $\left\{E_{i}, G_{i}^{ \pm 1}\right\}$.

The proof is by induction first on the number $l$ of simple windings of $T$ on non-closed strands, and then on the number $K$ of crossings of ordinary strands of $T$.

Suppose that $T$ has no simple windings on non-closed strands. Because $T$ is totally descending and all simple windings are located on closed loops of $T, T$ is regularly isotopic to a diagram in which the closed loops are confined to a neighborhood of the flagpole and the non-closed strands do not enter this neighborhood. It follows that $T \in \widehat{\mathrm{KT}}_{0, S} \otimes K T_{n, S}$, which is isomorphic to $K T_{n, S}$ by Turaev's theorem [19]. By Theorem 5.20, $T$ is in the subalgebra generated by $\left\{E_{i}, G_{i}^{ \pm 1}\right\}$.

Suppose $T$ has $l \geq 1$ simple windings on non-closed strands and that any totally descending affine tangle diagram in standard position with fewer than $l$ simple windings on non-closed strands is in the subalgebra of $\widehat{\mathrm{KT}}_{n, S}$ generated by $X_{1}^{ \pm 1}$ and $\left\{E_{i}, G_{i}^{ \pm 1}\right\}$. If the number $K$ of crossings of ordinary strands is zero, then by Lemma $6.4, T=A T_{0}$ or $T=T_{0} A$, where $A$ is in the monoid generated by $\left\{X_{1}^{ \pm 1}, G_{i}^{ \pm 1}\right\}$, and $T_{0}$ is an affine tangle diagram in standard position with $l-1$ simple windings. By the induction assumption on $l$, the affine tangle $T_{0}$ and hence $T$ is in the subalgebra of $\widehat{\mathrm{KT}}_{n, S}$ generated by $X_{1}^{ \pm 1}$ and $\left\{E_{i}, G_{i}^{ \pm 1}\right\}$.

Now suppose $T$ has $l \geq 1$ simple windings on non-closed strands and $K \geq 1$ crossings of ordinary strands. Then, by Lemma $6.4, T$ can be written as $T=A T_{0}+B$ or $T=T_{0} A+B$ where $B$ is has fewer than $K$ crossings, $T_{0}$ has fewer than $l$ simple windings, and $A$ is in the monoid generated by $\left\{X_{1}^{ \pm 1}, G_{i}^{ \pm 1}\right\}$. It follows from the induction assumptions on $l$ and $K$ that $T$ is in the subalgebra of $\widehat{\mathrm{KT}}_{n}$ generated by $X_{1}^{ \pm 1}$ and $\left\{E_{i}, G_{i}^{ \pm 1}\right\}$.

6.2. Freeness of $\widehat{W}_{n}$ and injectivity of $\varphi: \widehat{W}_{n} \rightarrow \widehat{\mathrm{KT}}_{n}$. Fix a ring $S$ with distinguished elements $\lambda, z, \delta$, and $q_{r}(r \geq 1)$. Write $W_{n}$ for $W_{n, S}$, $\mathrm{KT}_{n}$ for $\mathrm{KT}_{n, S}, \widehat{W}_{n}$ for $\widehat{W}_{n, S}$, and $\widehat{\mathrm{KT}}_{n}$ for $\widehat{\mathrm{KT}}_{n, S}$. 
We can identify $W_{n}$ with $\mathrm{KT}_{n}$ via $\varphi$, and we can regard $W_{n}$ as imbedded in $\widehat{W}_{n}$ by Corollary 5.45 and $\mathrm{KT}_{n}$ imbedded in $\widehat{\mathrm{KT}}_{n}$ by Remark 2.11 .

Lemma 6.6. Let $T^{\prime} \in \mathrm{KT}_{n}$ be a totally descending $(n, n)$-tangle diagram whose strands have no self-crossings. Suppose that $T^{\prime}$ has a strand connecting a top vertex $\boldsymbol{a}$ with a bottom vertex $\overline{\boldsymbol{b}}$, and that this strand lies above all other strands (i.e. has only over-crossings with other strands). Let $T^{\prime \prime}$ be the otherwise identical tangle diagram in which the strand connecting $\boldsymbol{a}$ and $\overline{\boldsymbol{b}}$ lies under all other strands. Then

$$
x_{b} \varphi^{-1}\left(T^{\prime}\right)=\varphi^{-1}\left(T^{\prime \prime}\right) x_{a} .
$$

Proof. If $T^{\prime}$ is a braid diagram, then the assertion follows from Propositions 3.10 and 3.11 and induction on the number of crossings.

Otherwise, $T^{\prime}$ is regularly isotopic to a tangle diagram of the form

$$
T^{\prime}=B_{1}\left[\left(E_{1} E_{3} \cdots E_{2 k-1}\right) \otimes B\right] B_{2},
$$

where $B_{1}, B_{2}$, and $B$ are totally descending braid diagrams, as described in the proof of Theorem 5.20. The $x_{*}$ can be successively passed through $\varphi^{-1}\left(B_{1}\right), \varphi^{-1}(B)$, and $\varphi^{-1}\left(B_{2}\right)$, by the result for braid diagrams.

REMark 6.7. Applying $\varphi$, we get

$$
X_{b} T^{\prime}=T^{\prime \prime} X_{a} .
$$

This statement can also be verified by the following picture proof (which provides the motivation for Lemma 6.6):
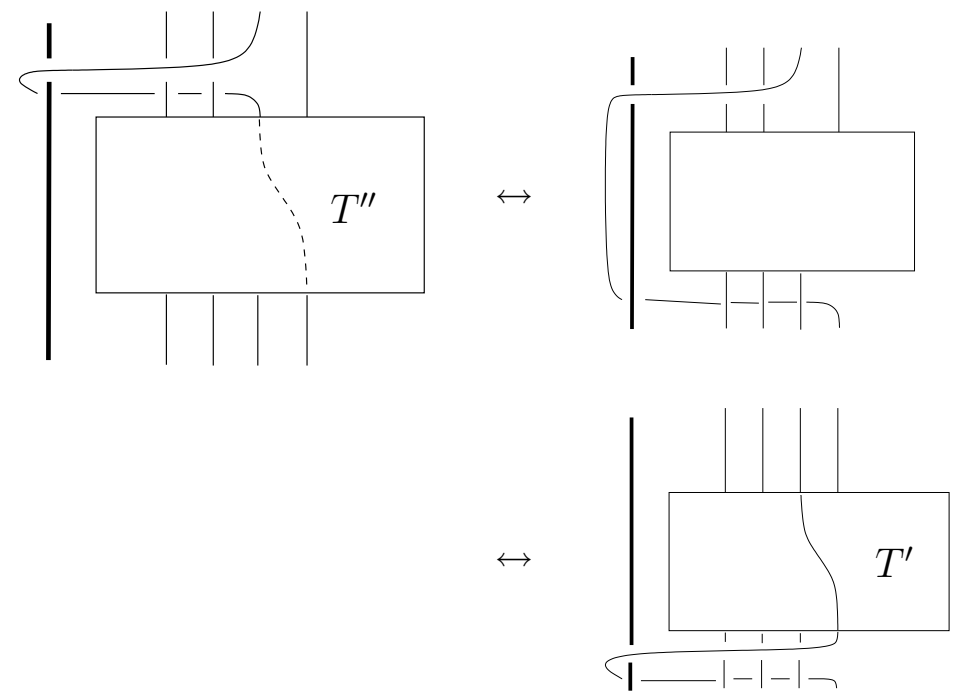

Lemma 6.8. Let $T^{\prime} \in \mathrm{KT}_{n}$ be a totally descending $(n, n)$-tangle diagram whose strands have no self-crossings. Suppose that $T^{\prime}$ has a strand connecting a bottom vertex $\overline{\boldsymbol{a}}$ with a bottom vertex $\overline{\boldsymbol{b}}$, and that this strand lies above all 
other strands (i.e. has only over-crossings with other strands). Let $T^{\prime \prime}$ be the otherwise identical tangle diagram in which the strand connecting $\overline{\boldsymbol{a}}$ and $\bar{b}$ lies under all other strands. Then

$$
x_{a} \varphi^{-1}\left(T^{\prime}\right)=\lambda^{-2} x_{b}^{-1} \varphi^{-1}\left(T^{\prime \prime}\right) .
$$

Proof. Assume without loss of generality that $a<b$. As in the proof of Lemma 5.38, $T^{\prime}$ has a factorization $T^{\prime}=G_{a} G_{a+1} \cdots G_{b-2} E_{b-1} T_{0}$. Thus

$$
\begin{aligned}
x_{a} \varphi^{-1}\left(T^{\prime}\right) & =x_{a} g_{a} \cdots g_{b-2} e_{b-1} \varphi^{-1}\left(T_{0}\right) \\
& =g_{a}^{-1} \cdots g_{b-2}^{-1} x_{b-1} e_{b-1} \varphi^{-1}\left(T_{0}\right) \\
& =\lambda^{-2} g_{a}^{-1} \cdots g_{b-2}^{-1} x_{b}^{-1} e_{b-1} \varphi^{-1}\left(T_{0}\right) \\
& =\lambda^{-2} x_{b}^{-1} g_{a}^{-1} \cdots g_{b-2}^{-1} e_{b-1} \varphi^{-1}\left(T_{0}\right) \\
& =\lambda^{-2} x_{b}^{-1} \varphi^{-1}\left(T^{\prime \prime}\right) .
\end{aligned}
$$

REMARK 6.9. Applying $\varphi$, we get

$$
X_{a} T^{\prime}=\lambda^{-2} X_{b}^{-1} T^{\prime \prime}
$$

This statement can also be verified by the following picture proof (which provides the motivation for Lemma 6.8):
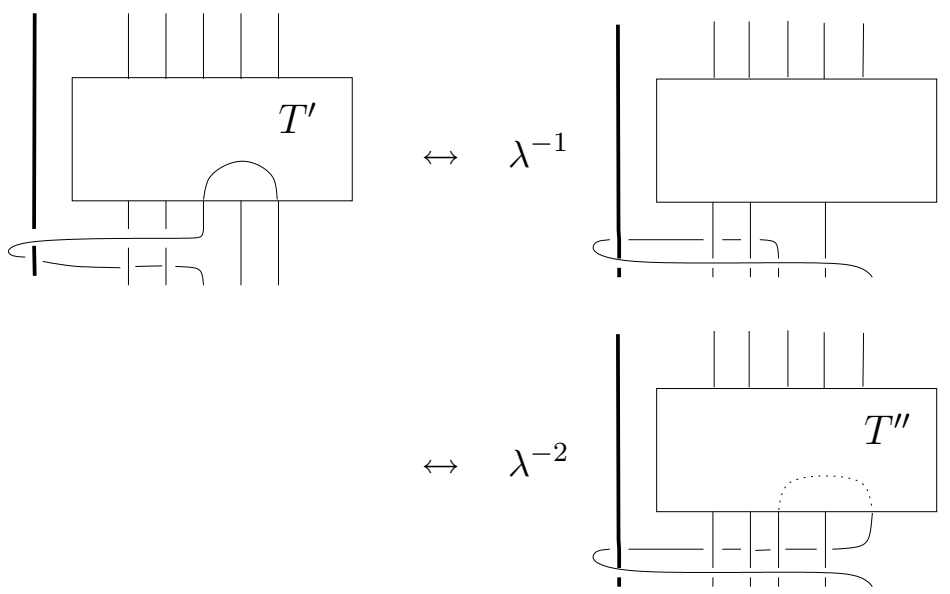

In the following we write $x^{\mu}=x_{1}^{\mu_{1}} \cdots x_{n}^{\mu_{n}}$ for a Laurent monomial in the commuting elements $x_{i}$.

Lemma 6.10 (Transport Lemma). Let $T \in \mathrm{KT}_{n}$ be an ordinary $(n, n)$ tangle diagram.

(1) Suppose $T$ has a strand connecting a top vertex $\boldsymbol{a}$ with a bottom vertex $\overline{\boldsymbol{b}}$. Then

$$
x_{b} \varphi^{-1}(T) \equiv \varphi^{-1}(T) x_{a}, \quad x_{b}^{-1} \varphi^{-1}(T) \equiv \varphi^{-1}(T) x_{a}^{-1}
$$


modulo the span of elements of the form $x^{\mu} \varphi^{-1}\left(T_{0}\right) x^{\nu}$, where $T_{0}$ is an ordinary $(n, n)$-tangle diagram with fewer crossings than $T$.

(2) Suppose $T$ has a strand connecting two bottom vertices $\overline{\boldsymbol{a}}$ and $\overline{\boldsymbol{b}}$. Then

$$
x_{a} \varphi^{-1}(T) \equiv \lambda^{-2} x_{b}^{-1} \varphi^{-1}(T)
$$

modulo the span of elements of the form $x^{\mu} \varphi^{-1}\left(T_{0}\right) x^{\nu}$, where $T_{0}$ is an ordinary $(n, n)$-tangle diagram with fewer crossings than $T$.

(3) Suppose $T$ has a strand connecting two top vertices $\boldsymbol{a}$ and $\boldsymbol{b}$. Then

$$
\varphi^{-1}(T) x_{a} \equiv \lambda^{-2} \varphi^{-1}(T) x_{b}^{-1}
$$

modulo the span of elements of the form $x^{\mu} \varphi^{-1}\left(T_{0}\right) x^{\nu}$, where $T_{0}$ is an ordinary $(n, n)$-tangle diagram with fewer crossings than $T$.

Proof. By the Kauffman skein relation, if $T^{\prime}$ is an ordinary $(n, n)$-tangle diagram which differs from $T$ by changing one or more crossings, then $T$ and $T^{\prime}$ are congruent modulo the span of $(n, n)$-tangle diagrams with fewer crossings.

Let $T^{\prime}$ be a diagram obtained from $T$ by changing crossings, in which the strand connecting $\boldsymbol{a}$ and $\bar{b}$ has only over-crossings with other strands, and let $T^{\prime \prime}$ be the otherwise identical diagram in which the strand connecting $\boldsymbol{a}$ and $\bar{b}$ has only under-crossings with other strands. Then $T \equiv T^{\prime} \equiv T^{\prime \prime}$ modulo the span of tangle diagrams with fewer crossings. By Lemma 6.6, $x_{b} \varphi^{-1}\left(T^{\prime}\right)=\varphi^{-1}\left(T^{\prime \prime}\right) x_{a}$, and the first assertion in statement (1) follows. The second assertion results from multiplying the congruence on the left by $x_{b}^{-1}$ and on the right by $x_{a}^{-1}$.

Statement (2) follows similarly using Lemma 6.6, and statement (3) is obtained from (2) by applying the anti-automorphism $\alpha$.

Proposition 6.11. $\widehat{W}_{n}$ is spanned by elements of the form $x^{\mu} w x^{\nu}$, where $x^{\mu}, x^{\nu}$ are Laurent monomials in the elements $x_{i}$ and $w \in W_{n}$.

Proof. Let $M$ denote the span of elements of the form $x^{\mu} w x^{\nu}$. Because $M$ contains the identity element, it suffices to show that $M$ is a left ideal in $\widehat{W}_{n}$.

Since $\widehat{W}_{n}$ is generated as a unital algebra by $x_{1}^{ \pm 1}$ and $\left\{g_{i}^{ \pm 1}, e_{i}\right\}$, it suffices to show that $M$ is invariant under left multiplication by these generators. Invariance under left multiplication by $x_{1}^{ \pm 1}$ is obvious (since the $x_{i}$ 's commute). Because of Propositions 3.10 and 3.11, invariance under left multiplication by $g_{i}^{ \pm 1}$ will follow from invariance under left multiplication by $e_{i}$.

For $k \geq 0$, let $M_{k}$ be the span of elements of the form $x^{\mu} \varphi^{-1}(T) x^{\nu}$, where $x^{\mu}, x^{\nu}$ are monomials in the elements $x_{i}$ and $T$ is an ordinary $(n, n)$ tangle diagram with no more than $k$ crossings. Let $M_{-1}=(0)$. We show by induction first on $i$ and then on $k$ that $e_{i} M_{k} \subseteq M$. 
Suppose $T$ is an ordinary $(n, n)$-tangle diagram with no more than $k$ crossings and $\overline{\boldsymbol{b}}$ and $\boldsymbol{a}$ are connected by a strand of $T$. Then, by Lemma 6.10, $x_{b} \varphi^{-1}(T) \equiv \varphi^{-1}(T) x_{a}$ and $x_{b}^{-1} \varphi^{-1}(T) \equiv \varphi^{-1}(T) x_{a}^{-1}$ modulo $M_{k-1}$. In particular, if $k=0$, then $x_{b} \varphi^{-1}(T)=\varphi^{-1}(T) x_{a}$ and $x_{b}^{-1} \varphi^{-1}(T)=$ $\varphi^{-1}(T) x_{a}^{-1}$. Likewise, if $\overline{\boldsymbol{b}}$ and $\overline{\boldsymbol{a}}$ are connected by a strand of $T$, then $x_{b} \varphi^{-1}(T) \equiv \lambda^{-2} x_{a}^{-1} \varphi^{-1}(T)$ modulo $M_{k-1}$, and in particular, if $k=0$, then $x_{b} \varphi^{-1}(T)=\lambda^{-2} x_{a}^{-1} \varphi^{-1}(T)$.

It follows from these observations that, for any $i$,

$$
x^{\mu} \varphi^{-1}(T) \equiv \varrho x^{\mu^{\prime}} \varphi^{-1}(T) x^{\nu^{\prime}} \bmod M_{k-1},
$$

where $\varrho \in S$ and

(1) if $\overline{\boldsymbol{i}}$ and $\overline{\boldsymbol{i}+\mathbf{1}}$ are not connected by a strand of $T$, then $\mu_{i}^{\prime}=\mu_{i+1}^{\prime}=0$,

(2) if $\overline{\boldsymbol{i}}$ and $\overline{\boldsymbol{i}+\mathbf{1}}$ are connected by a strand of $T$, then $\mu_{i+1}^{\prime}=0$.

For all $i$, if $\overline{\boldsymbol{i}}$ and $\overline{\boldsymbol{i}+\mathbf{1}}$ are not connected by a strand of $T$, then

$$
e_{i} x^{\mu} \varphi^{-1}(T) \equiv \varrho e_{i} x^{\mu^{\prime}} \varphi^{-1}(T) x^{\nu^{\prime}}=\varrho x^{\mu^{\prime}} e_{i} \varphi^{-1}(T) x^{\nu^{\prime}} \bmod e_{i} M_{k-1} .
$$

Suppose $\overline{\boldsymbol{i}}$ and $\overline{\boldsymbol{i}+\mathbf{1}}$ are connected by a strand $s$ of $T$. We have

$$
e_{i} x^{\mu} \varphi^{-1}(T) \equiv \varrho e_{i} x^{\mu^{\prime}} x_{i}^{r} \varphi^{-1}(T) \bmod e_{i} M_{k-1}=\varrho x^{\mu^{\prime}} e_{i} x_{i}^{r} \varphi^{-1}(T),
$$

where $\mu_{i}^{\prime}=\mu_{i+1}^{\prime}=0$. Modulo the span of diagrams with fewer crossings, $T$ is congruent to a totally descending tangle diagram $T^{\prime}$. Moreover, $T^{\prime}=E_{i} T^{\prime \prime}$ (where $T^{\prime \prime}$ may have more than $k$ crossings). In fact, $T^{\prime}$ must have a strand $s^{\prime}$ connecting two points on the top boundary of $\mathbb{R} \times I$. By regular isotopy, $s$ can be contracted and $s^{\prime}$ pulled down so that the minimum on $s^{\prime}$ lies just above the maximum on $s$ and both lie below any other crossing or local extremum of the tangle diagram. Thus

$$
\begin{aligned}
e_{i} x^{\mu} \varphi^{-1}(T) & \equiv \varrho x^{\mu^{\prime}} e_{i} x_{i}^{r} e_{i} \varphi^{-1}\left(T^{\prime \prime}\right) \bmod e_{i} M_{k-1} \\
& =\varrho x^{\mu^{\prime}} b e_{i} \varphi^{-1}\left(T^{\prime \prime}\right),
\end{aligned}
$$

where $b \in \widehat{W}_{n-1}$, by Proposition 3.17. It follows from equations (6.1) and (6.3) that for all $i$ and $k$,

$$
e_{i} M_{k} \subseteq M_{k}+e_{i} M_{k-1}+A \widehat{W}_{i-1} M,
$$

where $A$ is the algebra of Laurent polynomials in $\left\{x_{j}: 1 \leq j \leq n\right\}$. For $i=1$, we have

$$
e_{1} M_{k} \subseteq M_{k}+e_{1} M_{k-1} .
$$

It follows by induction on $k$ that $e_{1} M_{k} \subseteq M_{k}$ for all $k$; that is, $e_{1} M \subseteq M$. Now fix $i>1$ and assume that $e_{j} M \subseteq M$ for all $j<i$; it follows that $\widehat{W}_{i-1} M \subseteq M$, and thus from (6.4), for all $k$,

$$
e_{i} M_{k} \subseteq M+e_{i} M_{k-1} .
$$

By induction on $k, e_{i} M_{k} \subseteq M$ for all $k$. 
Let $B_{0}=\left\{T_{d}: d\right.$ is an $n$-connector $\}$ be the common basis of $\mathrm{KT}_{n, R}$ for all $R$, as described at the end of Section 5.1. Let $B$ be the set of elements $X^{\mu} T X^{\nu}$, where $T \in B_{0}$ and $\nu_{i}=0$ unless $T$ has a strand with initial point $\boldsymbol{i}$, and $\mu_{j}=0$ unless $T$ has a strand with initial point $\overline{\boldsymbol{j}}$. The set $B$ may be regarded as a subset of $\widehat{\mathrm{KT}}_{n, S}$ for any $S$.

For any $S$, we lift $B$ to a subset of $\widehat{W}_{n, S}$ as follows: $\varphi: W_{n, S} \rightarrow \mathrm{KT}_{n, S}$ is an isomorphism. We take $A=A(n, S)$ to be the set of elements of $\widehat{W}_{n, S}$ of the form $x^{\mu} \varphi^{-1}(T) x^{\nu}$, where $T \in B_{0}$ and $\nu_{i}=0$ unless $T$ has a strand with initial point $\boldsymbol{i}$, and $\mu_{j}=0$ unless $T$ has a strand with initial point $\overline{\boldsymbol{j}}$.

Proposition 6.12. For every $S$ and $n$,

(1) $A(n, S)$ spans $\widehat{W}_{n, S}$.

(2) $B$ spans $\widehat{\mathrm{KT}}_{n, S}$.

Proof. Because of Proposition 6.11, to prove statement (1) it suffices to show that every element of the form $x^{\mu} \varphi^{-1}(T) x^{\nu}$, where $T$ is an ordinary $(n, n)$-tangle diagram, is in the span of $A=A(n, S)$. The basis $B_{0}$ has the following triangular property: any ordinary $(n, n)$-tangle diagram is congruent to a multiple of an element of $B_{0}$ modulo the span of diagrams with fewer crossings. Combining this with the Transport Lemma 6.10, we can prove that $x^{\mu} \varphi^{-1}(T) x^{\nu}$ is in the span of $A$ by induction on the number of crossings of $T$.

Statement (2) follows from statement (1) by applying the surjective map $\varphi: \widehat{W}_{n, S} \rightarrow \widehat{\mathrm{KT}}_{n, S}$.

TheOREM 6.13. For all $n$,

(1) $\widehat{W}_{n, \widehat{\Lambda}}$ is a free $\widehat{\Lambda}$-module with basis $A$.

(2) $\widehat{\mathrm{KT}}_{n, \widehat{\Lambda}}$ is a free $\widehat{\Lambda}$-module with basis $B$.

(3) $\varphi: \widehat{W}_{n, \widehat{\Lambda}} \rightarrow \widehat{\mathrm{KT}}_{n, \widehat{\Lambda}}$ is an isomorphism.

Proof. $A=A(n, \widehat{\Lambda})$ spans $\widehat{W}_{n, \widehat{\Lambda}}$, and $B=\varphi(A)$ spans $\widehat{\mathrm{KT}}_{n, \widehat{\Lambda}}$, by Proposition 6.12. Since $c$ is injective on $B$, Proposition 4.7 implies that $B$ is linearly independent over $\widehat{\Lambda}$. All the conclusions follow.

Corollary 6.14.

(1) $\widehat{\mathrm{KT}}_{n, S}$ is a free $S$ module with basis $B$ and $\widehat{\mathrm{KT}}_{n, S} \cong \widehat{\mathrm{KT}}_{n, \widehat{\Lambda}} \otimes_{\widehat{\Lambda}} S$.

(2) $\varphi: \widehat{W}_{n, S} \rightarrow \widehat{\mathrm{KT}}_{n, S}$ is an isomorphism.

(3) $\widehat{W}_{n, S} \cong \widehat{W}_{n, \widehat{\Lambda}} \otimes_{\widehat{\Lambda}} S$.

Proof. By Proposition $6.12, B$ spans $\widehat{\mathrm{KT}}_{n, S}$. On the other hand, $B \otimes 1$ is a basis of $\widehat{\mathrm{KT}}_{n, \widehat{\Lambda}} \otimes_{\widehat{\Lambda}} S$. Moreover, we have an $S$-algebra homomorphism 
from $\widehat{\mathrm{KT}}_{n, S}$ to $\widehat{\mathrm{KT}}_{n, \widehat{\Lambda}} \otimes_{\widehat{\Lambda}} S$ which takes a tangle $T$ to $T \otimes 1$. Since this map takes a spanning set to a basis, it is an isomorphism. This proves (1).

Once we know that $B$ is a basis of $\widehat{\mathrm{KT}}_{n, S}$, it follows at once that $\varphi$ : $\widehat{W}_{n, S} \rightarrow \widehat{\mathrm{KT}}_{n, S}$ is an isomorphism. Namely, $\varphi$ carries the spanning set $A(n, S)$ to the basis $B$, and so is an isomorphism.

Finally, (3) follows by combining the isomorphisms

$$
\widehat{W}_{n, S} \cong \widehat{\mathrm{KT}}_{n, S}, \quad \widehat{\mathrm{KT}}_{n, S} \cong \widehat{\mathrm{KT}}_{n, \widehat{\Lambda}} \otimes_{\widehat{\Lambda}} S, \quad \widehat{W}_{n, \widehat{\Lambda}} \otimes_{\widehat{\Lambda}} S \cong \widehat{\mathrm{KT}}_{n, \widehat{\Lambda}} \otimes_{\widehat{\Lambda}} S .
$$

Corollary 6.15. The map $\iota: \widehat{W}_{n-1, S} \rightarrow \widehat{W}_{n, S}$ is injective.

Corollary 6.16 .

(1) For each $n \geq 1$, there is a conditional expectation $\varepsilon_{n}: \widehat{W}_{n, S} \rightarrow$ $\widehat{W}_{n-1, S}$ satisfying

$$
e_{n} x e_{n}=\delta \varepsilon_{n}(x) e_{n}
$$

for $x \in \widehat{W}_{n, S}$.

(2) There is a trace $\varepsilon: \widehat{W}_{n, S} \rightarrow S$ defined by

$$
\varepsilon=\varepsilon_{1} \circ \cdots \circ \varepsilon_{n} .
$$

(3) The trace $\varepsilon$ has the Markov property: for $b \in \widehat{W}_{n-1, S}$,

(a) $\varepsilon\left(b g_{n-1}^{ \pm 1}\right)=\left(\lambda^{ \pm 1} / \delta\right) \varepsilon(x)$,

(b) $\varepsilon\left(b e_{n-1}\right)=(1 / \delta) \varepsilon(x)$,

(c) $\varepsilon\left(b\left(x_{n}^{\prime}\right)^{r}\right)=q_{r} \varepsilon(b)$ and $\varepsilon\left(b\left(x_{n}^{\prime}\right)^{-r}\right)=f_{r}\left(q_{1}, \ldots, q_{r}\right) \varepsilon(b)$ for $r \geq 1$, where $x_{n}^{\prime}=\left(g_{n-1} \cdots g_{1}\right) x_{1}\left(g_{1}^{-1} \cdots g_{n-1}^{-1}\right)$.

Proof. All statements follow from the isomorphism $\widehat{W}_{n, S} \cong \widehat{\mathrm{KT}}_{n}$ and the properties of $\widehat{\mathrm{KT}}_{n, S}$ discussed in Section 2.7.

REMARK 6.17. The trace $\varepsilon$ is the unique trace on $\widehat{W}_{n, S}$ satisfying $\varepsilon(1)=1$ and the Markov properties enumerated in statement (3) of the previous corollary. To prove this uniqueness statement, one needs an analogue of Proposition 3.16 with $x_{n}$ replaced by $x_{n}^{\prime}$.

Corollary 6.18. For $x \in \widehat{W}_{n+1}$,

$$
x e_{n}=\delta \varepsilon_{n+1}\left(x e_{n}\right) e_{n} .
$$

Proof. By Proposition 3.20, there exists a $y \in \widehat{W}_{n}$ such that $x e_{n}=y e_{n}$. Applying the conditional expectation $\varepsilon_{n+1}$ to both sides gives $\varepsilon_{n+1}\left(x e_{n}\right)=$ $y \varepsilon_{n+1}\left(e_{n}\right)=\delta^{-1} y$.

COROLlaRY 6.19. Every element of $\widehat{\mathrm{KT}}_{n, S}$ is a linear combination of elements of the form $a \chi b$, where $a, b \in \widehat{\mathrm{KT}}_{n-1, S}$ and

$$
\chi \in\left\{E_{n-1}, G_{n-1}^{ \pm 1}\right\} \cup\left\{X_{n}^{r}: r \in \mathbb{Z}\right\} .
$$


Proof. This follows from the isomorphism $\widehat{W}_{n, S} \cong \widehat{\mathrm{KT}}_{n, S}$ and Proposition 3.16 .

\section{References}

[1] S. Ariki, A. Mathas and H. B. Rui, Cyclotomic Nazarov-Wenzl algebras, arXiv:math. QA $/ 0506467,2005$.

[2] J. Birman and H. Wenzl, Braids, link polynomials and a new algebra, Trans. Amer. Math. Soc. 313 (1989), 249-273.

[3] R. Brauer, On algebras which are connected with the semisimple continuous groups, Ann. of Math. (2) 38 (1937), 857-872.

[4] R. Häring-Oldenburg, The reduced Birman-Wenzl algebra of Coxeter type B, J. Algebra 213 (1999), 437-466.

[5] —, Cyclotomic Birman-Murakami-Wenzl algebras, J. Pure Appl. Algebra 161 (2001), 113-144.

[6] V. Jones, Index for subfactors, Invent. Math. 72 (1983), 1-25.

[7] V. Jones and V. Sunder, Introduction to Subfactors, London Math. Soc. Lecture Note Ser. 234, Cambridge Univ. Press, Cambridge, 1997.

[8] L. Kauffman, An invariant of regular isotopy, Trans. Amer. Math. Soc. 318 (1990), $417-471$.

[9] H. Morton and P. Traczyk, Knots and algebras, in: E. Martín-Peinador and A. Rodez Usan (eds.), Contribuciones Matematicas en homenaje al profesor D. Antonio Plans Sanz de Bremond, Univ. of Zaragoza, Zaragoza, 1990, 201-220.

[10] H. Morton and A. Wassermann, A basis for the Birman-Wenzl algebra, unpublished manuscript, 1989, revised 2000, $29 \mathrm{pp}$.

[11] J. Murakami, The Kauffman polynomial of links and representation theory, Osaka J. Math. 24 (1987), 745-758.

[12] M. Nazarov, Young's orthogonal form for Brauer's centralizer algebra, J. Algebra 182 (1996), 664-693.

[13] A. Okounkov, On representations of the infinite symmetric group, Zap. Nauchn. Sem. POMI 240 (1997), 166-228 (in Russian); English transl.: J. Math. Sci. (New York) 96 (1999), 1550-3589.

[14] G. Olshanski, Unitary representations of $(G, K)$-pairs that are connected with the infinite symmetric group $S(\infty)$, Algebra i Analiz 1 (1989), no. 4, 178-209 (in Russian); English transl.: Leningrad Math. J. 1 (1990), 983-1014.

[15] R. Orellana and A. Ram, Affine braids, Markov traces and the category $O$, arXiv: math.RT/0401317, 2004.

[16] M. Pimsner and S. Popa, Iterating the basic construction, Trans. Amer. Math. Soc. 310 (1988), 127-133.

[17] J. Przytycki, Skein modules of 3-manifolds, Bull. Polish Acad. Sci. Math. 39 (1991), $91-100$.

[18] H. B. Rui and W. H. Yu, On the semi-simplicity of the cyclotomic Brauer algebras, J. Algebra 277 (2004), 187-221.

[19] V. Turaev, The Conway and Kauffman modules of a solid torus, Zap. Nauchn. Sem. Leningrad. Otdel. Mat. Inst. Steklov. (LOMI) 167 (1988) 79-89 (in Russian); English transl.: J. Soviet Math. 52 (1990), 2799-2805.

[20] H. Wenzl, On the structure of Brauer's centralizer algebras, Ann. of Math. (2) 128 (1988), 173-193. 
[21] H. Wenzl, Quantum groups and subfactors of type B, C, and D, Comm. Math. Phys. 133 (1990), 383-432.

Department of Mathematics

University of Iowa

Iowa City, IA 52242, U.S.A.

E-mail: goodman@math.uiowa.edu
Department of Mathematics University of California San Diego La Jolla, CA 92093, U.S.A. E-mail: hhauschild@math.ucsd.edu

Received 20 November 2004;

in revised form 23 December 2005 